\title{
Article
}

\section{Immature Dentate Granule Cells Require Ntrk2/ Trkb for the Formation of Functional Hippocampal Circuitry}

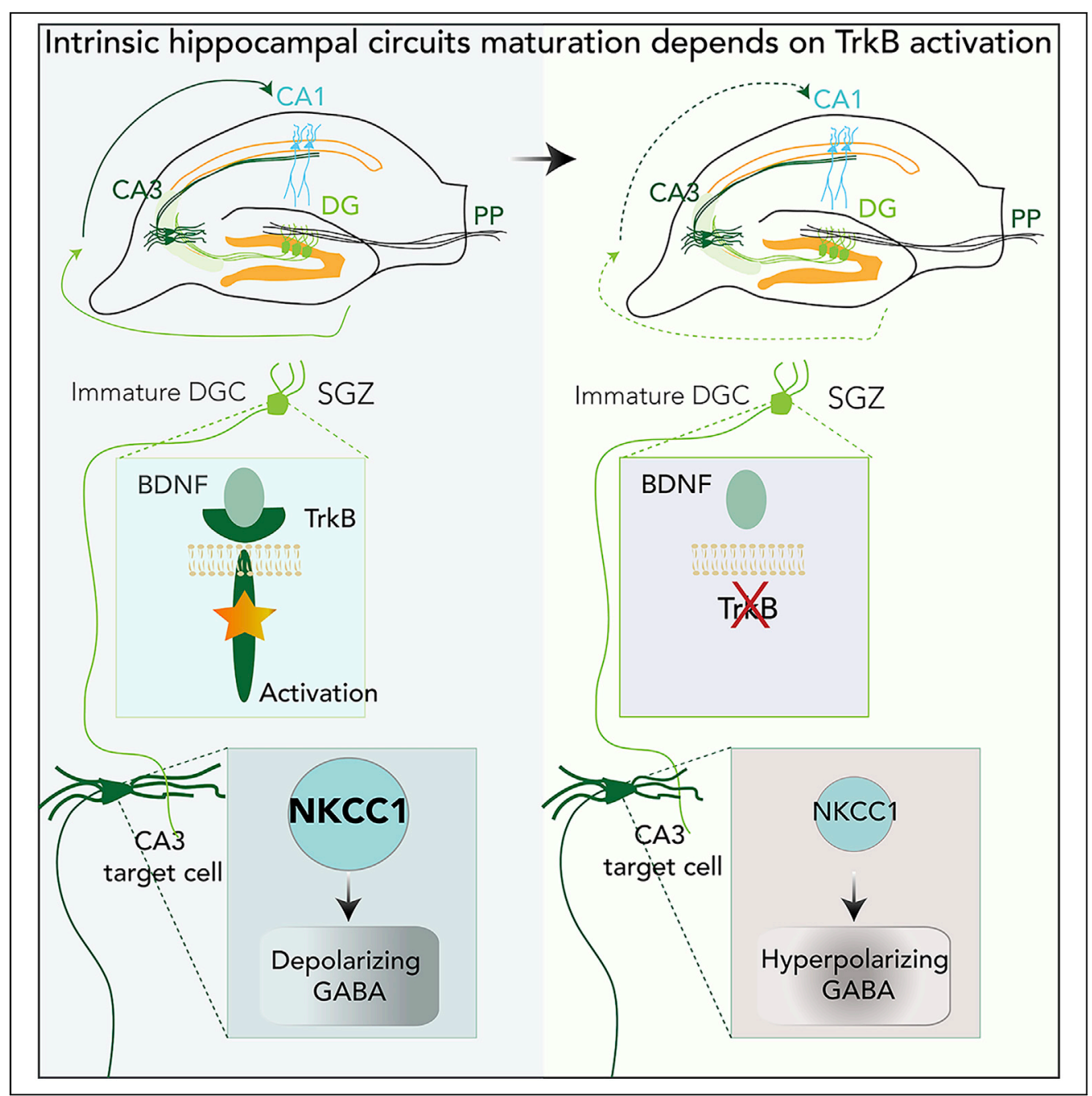

Sylvia Badurek, Marilena Griguoli, Aman Asif-

Malik, ..., José María DelgadoGarcía, Enrico Cherubini, Liliana Minichiello

liliana.minichiello@pharm.ox. ac.uk

HIGHLIGHTS

TrkB signaling shapes hippocampal circuitry by controlling early GABA signaling

Altered Nkcc1 expression reduces $C A 3$ pyramidal cell GABAergic excitatory drive

Early network activity is crucial for establishing functional hippocampal circuitry 


\title{
Article \\ Immature Dentate Granule Cells Require Ntrk2/Trkb for the Formation of Functional Hippocampal Circuitry
}

\author{
Sylvia Badurek, 2,3,9,12 Marilena Griguoli, ${ }^{4,12}$ Aman Asif-Malik, ${ }^{1,12}$ Barbara Zonta, ${ }^{1,12}$ Fei Guo, ${ }^{1}$ Silvia Middei, ${ }^{5}$ \\ Laura Lagostena, 6,10 Maria Teresa Jurado-Parras, ${ }^{7,11}$ Thomas H. Gillingwater, ${ }^{8}$ Agnès Gruart, ${ }^{7}$ \\ José María Delgado-García, ${ }^{7}$ Enrico Cherubini, 4,6 and Liliana Minichiello 1,2,3,13,*
}

\begin{abstract}
SUMMARY
Early in brain development, impaired neuronal signaling during time-sensitive windows triggers the onset of neurodevelopmental disorders. GABA, through its depolarizing and excitatory actions, drives early developmental events including neuronal circuit formation and refinement. BDNF/TrkB signaling cooperates with GABA actions. How these developmental processes influence the formation of neural circuits and affect adult brain function is unknown. Here, we show that early deletion of Ntrk2/Trkb from immature mouse hippocampal dentate granule cells (DGCs) affects the integration and maturation of newly formed DGCs in the hippocampal circuitry and drives a premature shift from depolarizing to hyperpolarizing GABAergic actions in the target of DGCs, the CA3 principal cells of the hippocampus, by reducing the expression of the cation-chloride importer Nkcc1. These changes lead to the disruption of early synchronized neuronal activity at the network level and impaired morphological maturation of CA3 pyramidal neurons, ultimately contributing to altered adult hippocampal synaptic plasticity and cognitive processes.
\end{abstract}

\section{INTRODUCTION}

Early in postnatal development, experience shapes neuronal circuits during time-sensitive periods. This process affects adult brain functions and leads to neurodevelopmental disorders arising from early dysfunction of neuronal signaling (Meredith et al., 2012). The hippocampus is a brain structure involved in several higher brain functions, including learning and memory, and spatial coding. The dentate gyrus (DG), input region of the hippocampus, plays a crucial role in these functions. Most dentate granule cells (DGCs) in the rodent brain are generated in the first postnatal week. The adult structure of the rodent DG, including the subgranular zone (SGZ), where neurons are continuously generated, is established between postnatal days (P) 7 and 14 (Nicola et al., 2015). Latest discoveries suggest that developmental and adult dentate neurogenesis are one continuous process whereby during embryonic development, dentate neural precursors generate DGCs to establish the primitive DG. They then adopt adult radial glial-like capacities in the SGZ during the early postnatal period and continue to generate DGCs in the adult thus resembling a unified process of extended development (Berg et al., 2019; Hochgerner et al., 2018).

Activity-dependent GABA-mediated excitation in the adult brain drives hippocampal progenitor cells from proliferation to neuroblast, migration, and integration of newly generated neurons in pre-existing functional circuits (Ge et al., 2006, 2007; Tozuka et al., 2005). This is due to the early depolarizing and excitatory effects of GABA on neural precursors and immature neurons exhibiting higher intracellular chloride concentration $\left[\mathrm{Cl}^{-}\right]$, which results from the differential temporal expression of the cation-chloride cotransporters NKCC1 and KCC2, involved in chloride uptake and extrusion, respectively (Ben-Ari, 2002; Ben-Ari et al., 2012; Owens and Kriegstein, 2002). In postnatal development, it has been shown that the premature shift of GABA from depolarizing to hyperpolarizing direction alters the morphological maturation of neonatal cortical neurons in vivo (Cancedda et al., 2007). Furthermore, in the immature hippocampus, the depolarizing action of GABA contributes to generate coherent network oscillations such as giant depolarizing potentials (GDPs), which represent a primordial form of synchrony between neurons that precedes more
1Department of

Pharmacology, University of Oxford, Oxford, United Kingdom

${ }^{2}$ Centre for

Neuroregeneration University of Edinburgh, Edinburgh, United Kingdom ${ }^{3}$ European Molecular Biology Laboratory, Mouse Biology Unit, Monterotondo, Rome, Italy

${ }^{4}$ European Brain Research Institute, Rome, Italy

${ }^{5}$ Institute of Cell Biology and Neurobiology, National Research Council,

Monterotondo, Rome, Italy

International School for

Advanced Studies (SISSA),

Department of

Neuroscience, Trieste, Italy

${ }^{7}$ Division of Neurosciences, University Pablo de Olavide, Seville, Spain

${ }^{8}$ Biomedical Sciences Edinburgh Medical School, University of Edinburgh,

Edinburgh, United Kingdom

${ }^{9}$ Present address: Vienna Biocenter Core Facilities GmbH (VBCF), 1030 Vienna, Austria

10present address: Institute of Biophysics (IBF-CNR), National Research Council, Genova, Italy

${ }^{11}$ Present address: Institut de Neurobiologie de la Méditerranée, INMED UMR901, Marseille, France

12These authors contributed equally

${ }^{13}$ Lead Contact

*Correspondence:

liliana.minichiello@pharm.ox. ac.uk

https://doi.org/10.1016/j.isci. 2020.101078 
organized forms of activity like theta and gamma rhythms. GDP-associated $\mathrm{Ca}^{2+}$ transients are instrumental in modifying synaptic efficacy at emerging GABAergic and glutamatergic synapses (Ben-Ari et al., 2012), contributing to the structural refinement of neuronal connectivity and the establishment of adult neural circuits. These are fundamental functions, and unsurprisingly, impaired GABAergic transmission gives rise to an array of neurodevelopmental disorders (Deidda et al., 2014). However, how these processes are triggered and influence adult brain function is unknown. GABAergic development relies highly on BDNF/ TrkB signaling (Gottmann et al., 2009; Hong et al., 2008). The latter is renowned for being one of the most critical regulators of glutamatergic and GABAergic synapse development and function in the developing and adult central nervous system (Cohen-Cory et al., 2010; Lu et al., 2005; Minichiello, 2009; Musumeci et al., 2009). Early in postnatal life, BDNF/TrkB signaling is instrumental in tuning hippocampal synaptic connections, in particular, at immature mossy fiber (MF)-CA3 synapses through the activation of the MAPK/ERK cascade (Mohajerani et al., 2007; Sivakumaran et al., 2009).

In this study, we asked whether BDNF/TrkB signaling would influence the establishment of hippocampal circuitry in vivo and eventually animal behavior in adulthood, by affecting the early depolarizing and excitatory actions of GABA. To answer this question, we used a novel genetic mouse model to remove TrkB signaling in immature DGCs early in postnatal development, coinciding with the integration time of these cells in the hippocampal circuitry. Here we show that such deletion affects the integration and maturation of newly formed DGCs in the forming DG. This, in turn, impairs the maturation of CA3 principal neurons via reduced expression of $N k c c 1$, with a consequent premature shift of GABA action from the depolarizing to the hyperpolarizing direction and GDP disruption, ultimately resulting in altered adult hippocampal synaptic plasticity and cognitive processes.

\section{RESULTS}

\section{Conditional Removal of Trkb in Immature Hippocampal Granule Cells}

Previously, we have shown that the BAC-Gad1-Cre mouse line expresses Cre-recombinase in DGCs within the hippocampal formation (Ohtsuka et al., 2013). To further characterize the hippocampal spatiotemporal expression pattern of this Cre-strain, we crossed the BAC-Gad1-Cre strain to different reporter lines (Z/EG, Rosa-YFP, and Rosa-Ai9-tdTomato) (Madisen et al., 2010; Novak et al., 2000). The analysis revealed GAD1Cre-reporter signal at P2 in the forming DG (Figure S1A), with increasing number of recombined granule cells at P7 (Figure S1B), and in adulthood where about 70\% of the total number of DAPI-stained DG nuclei were positive for the reporter signal (Figures S1E and S1H). Cell bodies in the CA3 and CA1 areas were devoid of fluorescent reporter signal in contrast with MFs, the DGCs' axons (Figure S1C, S1D, S1F, and S1G). Similarly, immunostaining for calcium-binding proteins, Parvalbumin (interneuron marker), Calretinin (interneuron and immature DGCs marker), and Calbindin (interneuron and mature DGCs marker) (von Bohlen Und Halbach, 2007) revealed no detectable overlapping staining between each primary antibody and tdTomato signal (BAC-Gad1-Cre ${ }^{\text {tg/+ }} ; \mathrm{Ai}^{T /+}$ ) in hippocampal interneurons (Figure S1I-S1O and insets). Colocalization was observed between tdTomato and Calretinin in immature DGCs of the SGZ (Figure S1P and inset), and consequently with Calbindin in the dentate granular cell layer (GCL) as they mature (Figure S1O and inset). To corroborate these results, we performed immunostaining using doublecortin (DCX), a protein expressed by neuronal precursor cells and immature neurons in the adult neurogenic brain areas (Brown et al., 2003). Expression of tdTomato colocalized specifically with DCX in immature DGCs beginning to migrate and mature into the GCL (Figure S1R). These findings are in agreement with a previous study showing a similar expression pattern in a transgenic line using the GAD1 promoter to drive EGFP (Cabezas et al., 2013). Cellular localization studies of the full-length TrkB has revealed its expression in many neuronal cell types of the hippocampus, including the DGCs and the pyramidal cells of the CA3 and CA1 regions as well as interneurons (Drake et al., 1999). Importantly, a recent transcriptome analysis using single dentate neural progenitors at different stages has revealed that Ntrk2/Trkb expression is upregulated from embryonic (E15.5) to early postnatal (P4) and adult (P45) stage (Figure S1S, data extracted from Berg et al., 2019). Therefore, given the specificity of the BAC-Gad1-Cre line in immature DGCs within the hippocampus, we crossed this Cre strain to the Trkb floxed strain (Minichiello et al., 1999) generating Trkb Gad1-KO mice to remove TrkB signaling from these cells (Figures S1T-S1W). This new strain allowed determining whether early GABA action requires BDNF/TrkB signaling at a critical period during development, coinciding with the integration time and maturation of newly born DGCs in the GCL, for the formation of functional hippocampal circuits. The Trkb Gad1-KO mice were viable and fertile and appeared hyperactive at around 3/4 weeks of age when handling for routine husbandry procedures. It was difficult to catch by hand; mutants would be faster to escape and run around the cage. This phenotype was less evident in adulthood. 

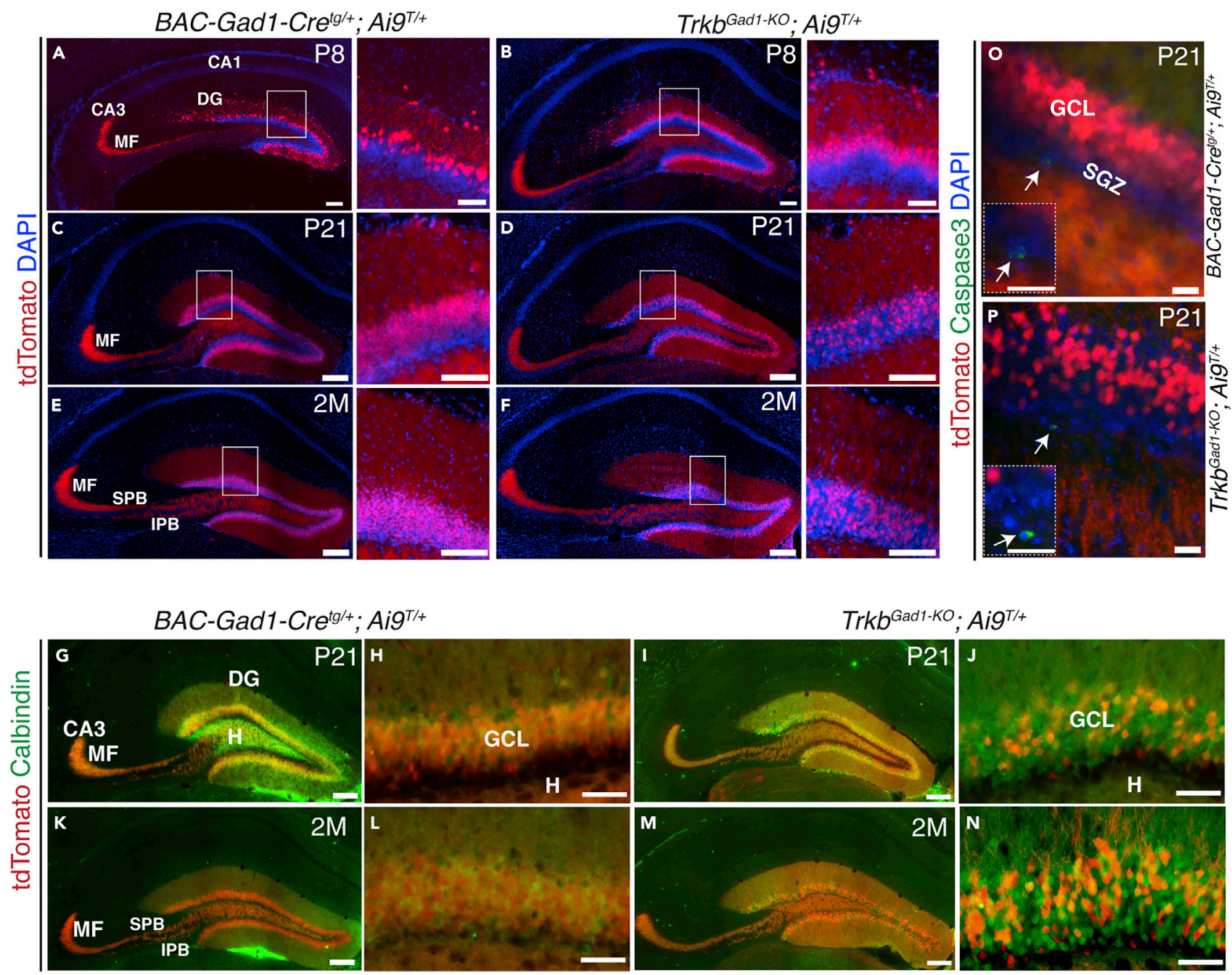

Figure 1. Time Course Analysis of the Dentate Gyrus Development in Trkb ${ }^{\text {Gad1-KO }}$ Mice and Controls

$(A-F)\left(A, C\right.$, and E) and (B, D, and F) Representative images of hippocampal coronal sections from controls (BAC-Gad1-Cre ${ }^{\text {tg/+ }} ;$ Aig $\left.^{T /+}\right)$ and mutants $\left(\right.$ Trkb Gad1- $^{-}$ $\mathrm{KO} ; A i \mathrm{G}^{T /+}$ ) at postnatal days 8 and 21 and 2 months (P8, P21, and $2 \mathrm{M}$ ), respectively, highlighting the forming dentate gyrus (DG) by tdTomato (red) endogenous fluorescence superimposed to DAPI staining (blue). Insets show higher magnification of the DG at all respective ages. Note the endogenous tdTomato fluorescence highlighting the distribution of mossy fiber bundles in control and Trkb Gad1-KO mice, namely the infrapyramidal mossy fiber (IPB) and the suprapyramidal mossy fiber (SPB) bundles, as well as the mossy fiber (MF) terminals targeting the hippocampal CA3 region.

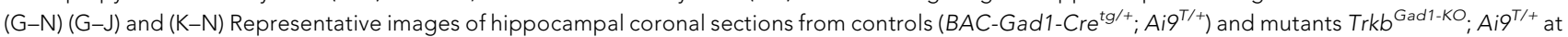
P21 and 2M, respectively, highlighting Calbindin immunostaining in mature dentate cells in the granule cell layer (GCL) and mossy fibers (MFs) targeting the CA3 region.

$(\mathrm{O}$ and $\mathrm{P})$ Representative images and respective insets showing caspase 3 immunofluorescence-stained (green) cells in the subgranular zone (SGZ) of both control $(\mathrm{O})$ and mutant $(\mathrm{P})$ mice indicated by a white arrow.

Scale bars: $100 \mu \mathrm{m}$ in (A and B) and $50 \mu \mathrm{m}$ in respective insets; $200 \mu \mathrm{m}$ in (C-F) and $100 \mu \mathrm{m}$ in respective insets; $200 \mu \mathrm{m}$ in (G, I, K, and M); $50 \mu \mathrm{m}$ in (H, J, L, and $\mathrm{N})$; and $25 \mu \mathrm{m}$ in (O and $\mathrm{P})$ and respective insets. See also Figure $\mathrm{S} 1$.

\section{Reduced CREB Activation and Affected Integration of Immature DGCs in Absence of TrkB Signaling}

To determine the effect of Trkb deletion in immature DGCs, we first performed a time course analysis of the DG development (P8, P21, and 2 months [2M]). Thus, the Ai9 reporter line was crossed to the Trkb Gad1-KO strain to be able to follow recombined cells. At P8 the forming GCL in the Trkb ${ }^{G_{a d 1}-K O} ; \mathrm{Ai}^{T /+}$ mice was comparable with controls, whereas at P21, and in the adult stage, fewer tdTomato-positive cells were integrated into the GCL of mutants compared with controls (Figures $1 \mathrm{~A}-1 \mathrm{~F}$ and insets). These data were corroborated by Calbindin staining, a marker of DGCs' maturity, showing a similar pattern (Figures $1 \mathrm{G}-1 \mathrm{~N}$ ). However, a cell 
BAC-Gad1-Cre $\mathrm{Crg}^{\mathrm{tg} /+} ; \mathrm{Aig}^{\mathrm{T} /+}$
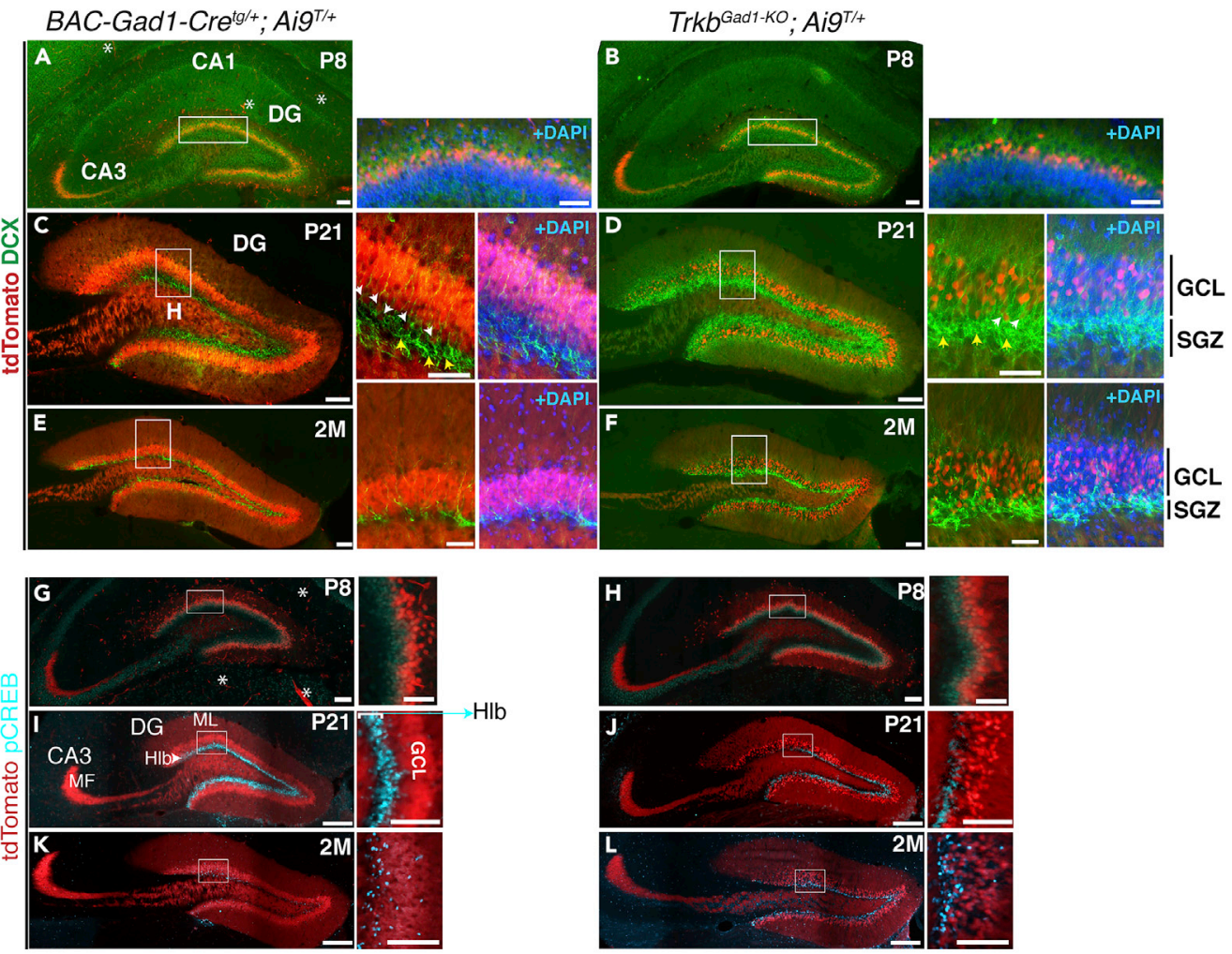

M
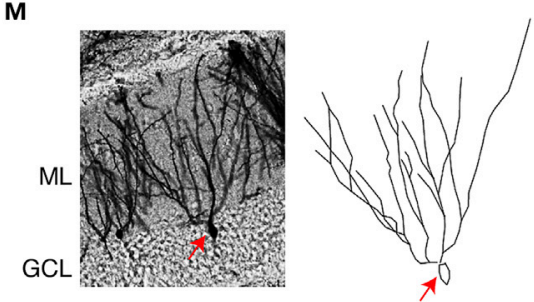

$\mathbf{N}$
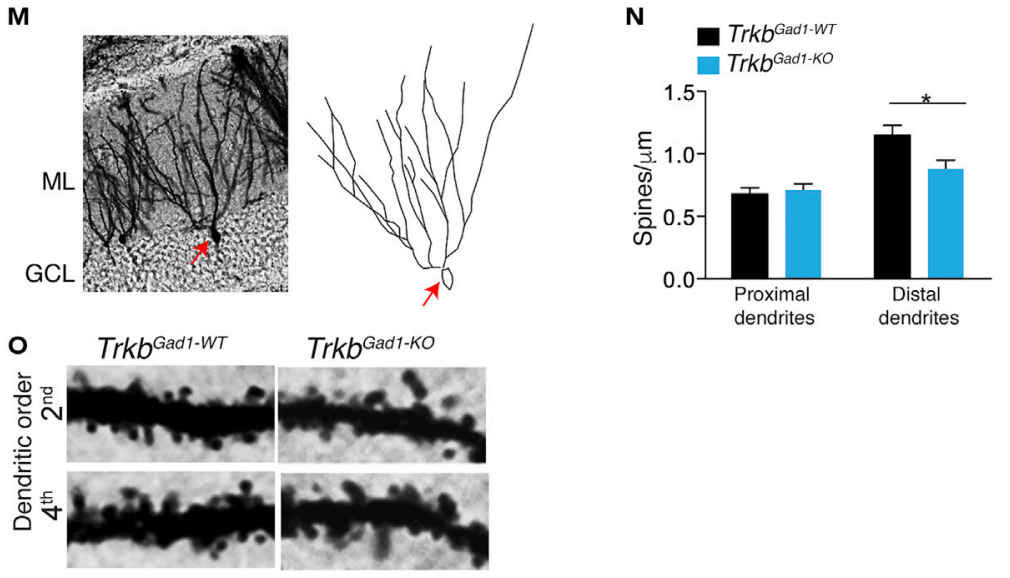

Figure 2. TrkB Signaling Regulates CREB Activation in Immature DGCs and the Integration and Maturation of These Cells into the GCL

(A-F) Immature DGCs accumulate at the hilar border in the absence of TrkB signaling. $(A, C$, and $E)$ and (B, D, and F) Representative images of hippocampal coronal sections from controls (BAC-Gad1-Cre ${ }^{\text {tg/+ }} ; A_{i 9^{T /+}}$ ) and mutants $\left(\right.$ Trkb ${ }^{\text {Gad1- }}$ $\left.\mathrm{KO} ; \mathrm{Ai}^{\mathrm{T} /+}\right)$, respectively, at $\mathrm{P} 8, \mathrm{P} 21$, and $2 \mathrm{M}$ immunostained for doublecortin (DCX) (green) and superimposed to tdTomato signal (red). At P8, DCX immunostaining shows mainly colocalization with endogenous tdTomato (red) indicating migrating DGCs and a similar pattern between controls and mutants ( $A$ and $B$ and insets). DAPI staining (blue). At P21, however, DCX immunostaining shows accumulation of immature DGCs in the subgranular zone (SGZ) of mutants compared with controls ( $C$ and $D$ and respective insets). At $2 \mathrm{M}$, as expected, very few DCX-positive DGCs are present in the SGZ of controls (E and insets), whereas in mutants the SGZ appears still abundant in DCX-positive cells, although reduced compared with P21 (F and insets). Scale bars: $100 \mu \mathrm{m}$ in ( $A$ and B) and respective insets; $100 \mu \mathrm{m}$ in (C-F) and $50 \mu \mathrm{m}$ in respective insets. White arrowheads indicate examples of colocalization between tdTomato and DCX occurring only in immature neuron DGCs beginning to migrate and mature in the GCL, but not in neural precursors (yellow arrows). (G-L) Representative images of hippocampal coronal sections and insets from controls (G, I, and K, BAC-Gad1-Cre ${ }^{\text {tg/+ }}$; $\left.A i 9^{T /+}\right)$ and mutants $\left(\mathrm{H}, \mathrm{J}\right.$, and $\left.\mathrm{L}, T r k b^{\mathrm{Gad} 1-K O} ; A i 9^{T /+}\right)$, respectively, at $\mathrm{P} 8, \mathrm{P} 21$, and $2 \mathrm{M}$ immunostained for phosphoCREB 
Figure 2. Continued

( $\mathrm{PCREB})$. The time course analysis shows PCREB peak of activation at P21 in control mice appearing in the lower third of the granule cell layer (GCL) at the hilar border ( $\mathrm{Hlb}$ ) where newborn immature neurons reside (I and inset). pCREB immunoreactivity declined by $2 \mathrm{M}$ age ( $\mathrm{K}$ and inset). Trkb deletion in immature DGCs induces reduced activation of CREB at P21 compared with controls ( $\mathrm{J}$ and $\mathrm{I}$ ) followed by a decline at $2 \mathrm{M}(\mathrm{L})$. Scale bars: $100 \mu \mathrm{m}$ in $(\mathrm{G}$ and $\mathrm{H})$ and $50 \mu \mathrm{m}$ in insets; $200 \mu \mathrm{m}$ in (I-L) and $100 \mu \mathrm{m}$ in insets. MF, mossy fibers; * examples of autofluorescence in structures like blood vessels.

(M-O) Golgi staining of newly formed DGCs to analyze dendritic density in the presence and absence of TrkB signaling. (M) Representative image of $2 \mathrm{M}$-old Golgi-stained granule cells in the GCL and drawing of the indicated (arrow) cell showing branching ramification. (N) A similar number of dendritic spines was found between genotypes in proximal dendrites (second to third order, Trkb ${ }^{\text {Gad-1-WT }}, 0.68 \pm 0.05 ; n=33$ cells from 4 mice; Trkb Gad-1-KO $0.71 \pm 0.05 ; n=34$ cells from 3 mice; $p=0.99$ ) of DGCs, whereas a significant reduction in dendritic spines was observed along distal dendrites in mutant mice compared with control littermate (fourth order, Trkb Gad1-WT, $1.15 \pm 0.08 ; \mathrm{n}=5$ cells from 4 mice; Trkb ${ }^{\text {Gad1-KO }}, 0.88 \pm 0.07 ; n=19$ cells from 3 mice; $\left.{ }^{*} p=0.02\right)$. Data are number of spines per 1 - $\mu$ m dendritic segment \pm SEM. (O) Representative images of $10-\mu \mathrm{m}$ dendritic segment of second and fourth order from mutants and control mice. See also Figure S2.

death marker caspase 3 (Porter and Janicke, 1999), for example, at P21, when the phenotype in the GCL is apparent, did not show increased apoptosis in mutants compared with control mice (Figures $1 \mathrm{O}$ and 1P). This was consistent with DAPI staining highlighting cell nuclei and a similar thickness of the GCL between mutants and controls at all ages analyzed (Figures $1 \mathrm{~A}-1 \mathrm{~F}$, and respective insets). The data suggested a delay in the migration and integration time of newly formed DGCs in the GCL rather than reduced cell survival. The presence of the forming MF bundles and their terminals targeting the hippocampal CA3 region as revealed by both tdTomato fluorescent signal (Figures 1A-1F) and Calbindin immunostaining (Figures 1G, 11, 1K, and $1 \mathrm{M})$ further supported this notion. Therefore, we performed a time course analysis for DCX immunostaining to specifically analyze immature DGCs beginning to migrate and integrate into the GCL. Although less evident at P8, at P21 there was an increase in DCX-positive (DCX+) cells in the SGZ of mutant compared with control mice (Figures 2A-2D, and respective insets). In control sections DCX immunofluorescence showed the typical progressive decrease with age (Figures $2 \mathrm{C}$ and $2 \mathrm{E}$ and insets), whereas in mutants there appeared to be a delay in the progression of immature DGCs into GCL supported by visibly less accumulated DCX + cells in the SGZ and more tdTomato + cells in the GCL at 2M (Figures 2D and 2F and insets). However, mutants at $2 \mathrm{M}$ still showed accumulation of DCX+ cells compared with controls (Figures 2D-2F and insets). These data were also supported by Ki67 immunostaining, a cell proliferation marker (Scholzen and Gerdes, 2000), showing similar proliferation of progenitors at different stages between mutants and controls (Figures S2A-S2F). Next, we asked how absence of TrkB signaling impairs integration of immature DGCs into the forming GCL. CREB signaling plays a key role in adult hippocampal neurogenesis by regulating the development and survival of newly generated immature DGCs downstream of GABA-mediated excitation (Jagasia et al., 2009). GABAergic development requires BDNF/TrkB signaling, as shown, for example, in the development of inhibition in the cortex (Hong et al., 2008). Therefore, we performed a time course analysis of CREB activation in the DG of Trkb ${ }^{\text {Gad1-KO}} ; \mathrm{Ai}^{T /+}$ mutant and control mice at age P8, P21, and 2M. This analysis revealed a peak of activation of CREB, measured through its phosphorylation ( $P C R E B$ ), at P21 in control mice. As reported previously for the adult DG (Jagasia et al., 2009), pCREB was predominantly detected in the lower third of the DGL at the hilar border where newborn immature neurons reside (Figures $2 \mathrm{G}$ and $2 \mathrm{l}$ and insets). PCREB immunoreactivity declined by $2 \mathrm{M}$ age (Figure $2 \mathrm{~K}$ and inset) coinciding with reduced neurogenesis. In the absence of TrkB signaling, however, there was reduced activation of CREB at P21 compared with controls followed by a decline at $2 \mathrm{M}$ (Figures $2 \mathrm{H}, 2 \mathrm{~J}$, and 2L). However, CREB activation at $2 \mathrm{M}$ was apparently higher in mutants than controls, reflecting the accumulation of DCX-positive cells at this age (Figures 2C-2F). To assess the maturation of the newly formed DGCs, we analyzed dendritic spine density of Golgistained granule cell in 2M-old mutant and control mice. The number of dendritic spines was similar between genotypes in proximal dendrites, whereas there was a significant reduction of dendritic spines along distal dendrites in Trkb Gad1-KO mice compared with control Trkb Gad1-WT (Figures 2M-2O). Thus, absence of TrkB signaling in immature DGCs reduces CREB activation, possibly through affected early GABA action, and delays the integration and maturation of these cells into the GCL.

\section{Deletion of Trkb from Immature DGCs Alters Coherent Network Oscillations and GABAergic Signaling in Developing CA3 Neurons}

We then asked what impact the absence of TrkB signaling in the immature DGCs would have on the establishment of hippocampal neural circuits. It is well known that the CA3 pyramidal cells and GABAergic interneurons in the hilus are the primary targets of MFs, the axons of DGCs that convey information from 
the entorhinal cortex (EC) to the hippocampus proper (Acsady et al., 1998). Moreover, although GDPs can be recorded from the entire hippocampus, their occurrence in the CA3 region is facilitated by the extensive network of excitatory collaterals and by the presence of intrinsic bursts that can drive other neurons to fire (Safiulina et al., 2008). Early in postnatal development, DGCs exhibit a mixed GABAergic/glutamatergic phenotype, and in addition to glutamate, MF can also release GABA (Safiulina et al., 2006). Thus, we first assessed the effect of Trkb loss in immature DGCs on coherent network oscillations in the developing CA3 area of the hippocampus. GDPs were recorded in current clamp conditions early in postnatal life (P3-P9) from Trkb Gad1-KO mice and controls. As expected (Ben-Ari et al., 2012), GDPs were characterized by recurrent GDPs with superimposed fast action potentials, separated by silent periods (Figure 3A). We observed a significant decrease in GDP frequency and the underlying area in Trkb ${ }^{\text {Gad1-KO }}$ mice compared with control littermates (Figure 3B). Several factors may contribute to the observed GDP disruption such as altered neuronal excitability in CA3 pyramidal neurons or early inhibitory action of GABA at the network level. To verify whether changes in cell excitability may contribute to GDP alterations, we measured spike frequency in response to depolarizing current pulses of increasing intensities, delivered at $-60 \mathrm{mV}$ membrane potential in the presence of $\mathrm{GABA}_{A}$ and AMPA/NMDA receptors blockers. No differences in firing threshold and frequencies were observed between the two genotypes (Figures S3A and S3B), suggesting that changes in neuronal excitability were not involved in GDP disruption. GDPs are generated within a local network by the interplay of GABA and glutamate, both depolarizing and excitatory (Ben-Ari et al., 2012). Thus, their impairment may also reflect alterations either in GABAergic or glutamatergic signaling. We recorded spontaneously, pharmacologically isolated $\mathrm{GABA}_{A^{-}}$-mediated postsynaptic currents (spontaneous $\mathrm{GABA}_{\mathrm{A}}$-mediated postsynaptic currents [sGPSCs], in the presence of DNQX, $20 \mu \mathrm{M}$, and DL-APV, $100 \mu \mathrm{M}$ ) and AMPA-mediated postsynaptic currents (spontaneous excitatory postsynaptic currents [sEPSCs], in the presence of DL-APV, $100 \mu \mathrm{M}$, and bicuculline, $10 \mu \mathrm{M}$ ) from P3 to P9 CA3 principal cells in both genotypes. Trkb Gad1-KO mice exhibited a significant increase in frequency, but not in amplitude, of sGPSCs compared with controls (Figures $3 \mathrm{C}$ and 3D). We obtained similar results for miniature events ( $m$ GPSCs) recorded in the presence of tetrodotoxin (TTX, $1 \mu \mathrm{M}$ ) (Figure 3C,3E, 3F). No significant changes in frequency and amplitude of spontaneous and miniature glutamatergic events (sEPSCs and mEPSCs) were detected between control and Trkb ${ }^{\text {Gad1-KO }}$ mice (Figures S4A-S4F). These results indicate that specifically perturbing presynaptic BDNF/TrkB signaling in immature DGCs at early postnatal age selectively affects GABAergic but not glutamatergic transmission in the downstream CA3 hippocampal area. Thus the altered GABAergic signaling may contribute to the disruption of GDPs.

\section{Increased GABAergic Synapses in CA3 Area of Trkb ${ }^{\text {Gad1-KO }}$ Mice}

The enhanced frequency of spontaneous and miniature GPSCs observed in Trkb ${ }^{\text {Gad1-KO }}$ mice may depend on an increased number of release sites and/or an increase in the probability of GABA release. We used electron microscopy (EM) and electrophysiology to measure the number of release sites and the probability of GABA release, respectively. In EM experiments we determined the number of "symmetric" (GABAergic) versus "asymmetric" (glutamatergic) synapses in the CA3 hippocampal area of Trkb Gad1-KO and control mice at P5 and young adult age. The total number of synapses was not significantly different between the two genotypes at P5 and young adult age (Figures 3 G and S4G), whereas we found a significant increase in the number of symmetric synapses in Trkb Gad1-KO mice compared with controls both at P5 and young adult age (Figures $3 \mathrm{H}$ and $\mathrm{S} 4 \mathrm{H}$ ). Besides, we observed a statistically significant decrease in the number of asymmetric synapses in Trkb Gad1-KO mice at P5 compared with control mice (Figure 3I). The reduced number of asymmetric synapses was not associated with a reduction of spontaneous EPSCs (Figure S4A) probably because when compared with GABAergic ones, at this developmental stage glutamatergic synapses may not be all functional (Durand et al., 1996). Conversely, in adult mice, the increase in symmetric synapses in mutants was not accompanied by a significant change in the number of asymmetric synapses (Figure S4I). No obvious gross morphological differences in the appearance of symmetric synapses between the two genotypes were noticed (Figures 3J-3L). In electrophysiological experiments, we determined the probability of GABA release from MF terminals measuring the paired-pulse ratio (PPR) among two pulses delivered to DGCs at 50-ms interval (in the presence of DNQX and DL-AP5 to block AMPA and NMDA receptors, respectively). In agreement with their MF origin (Safiulina et al., 2006), synaptic currents exhibited a strong paired-pulse facilitation and were significantly reduced by the mGluR agonist L-AP4 both in controls $(43.3 \% \pm 6.3 \%, \mathrm{n}=6 ; \mathrm{p}=0.03)$ and Trkb ${ }^{\text {Gad1-KO }}$ mice $(56.3 \% \pm 6.0 \%, \mathrm{n}=$ $8 ; p=0.008$, respectively; Wilcoxon test). We found similar values of PPR in both genotypes (Figures $3 \mathrm{M}$ and $3 N$ ) suggesting that changes in the probability of GABA release from DGCs do not contribute to the observed effects. Overall, these experiments revealed that the increase in GABAergic synapses is 


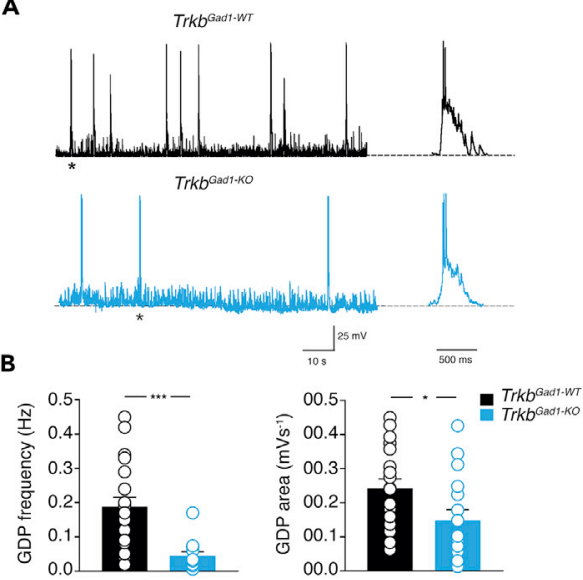

G $\quad$ P5

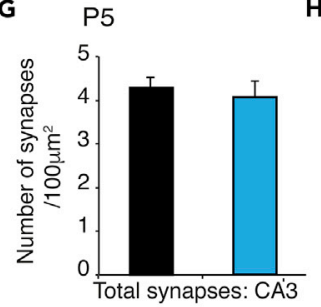

H
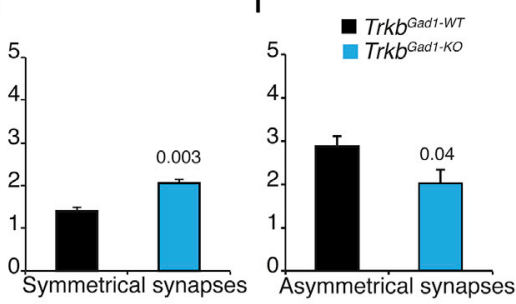

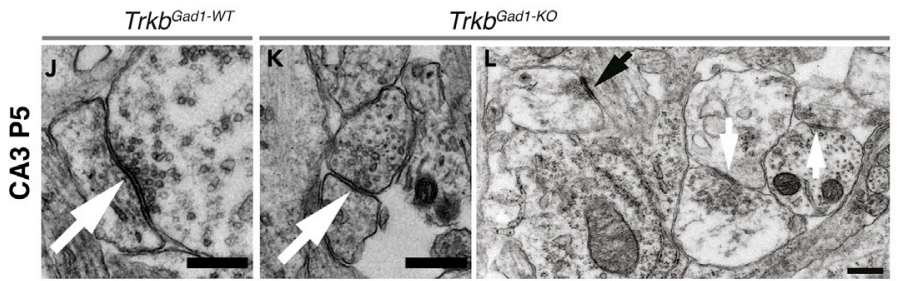

C

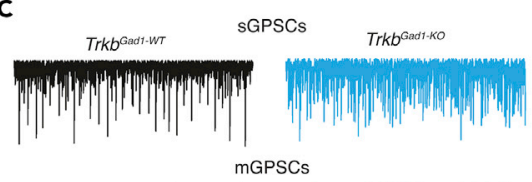

D

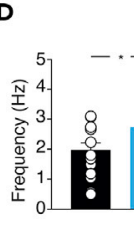

$\mathbf{F}$
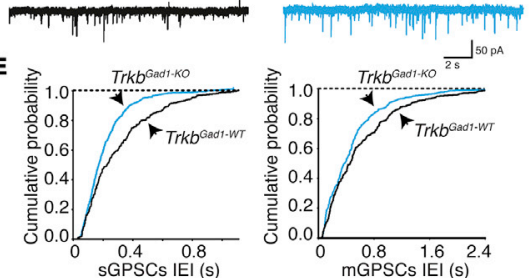

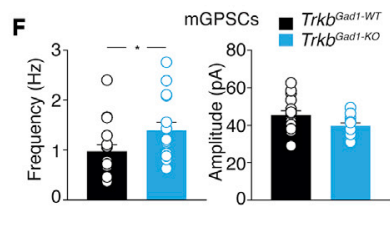

M

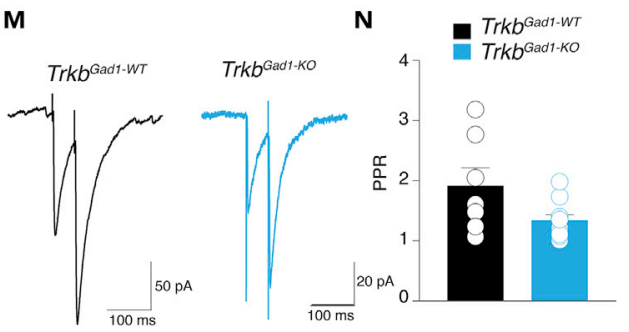

Figure 3. Reduced Expression of Coherent Network Oscillations in CA3 Hippocampal Neurons of Trkb ${ }^{\text {Gad1-KO }}$ Mice

(A) GDPs recorded from P3 to P9 Trkb ${ }^{G a d 1-W T}$ and Trkb ${ }^{\text {Gad1-KO }}$ mice. An expanded timescale for the GDPs marked with an asterisk is shown on the right. (B) Summary plots showing the mean frequency and the mean area of GDPs obtained from Trkb Gad1-WT and Trkb Gad1-KO mice; GDPs (frequency: control $0.019 \pm 0.003 \mathrm{~Hz}\left[\mathrm{n}=20\right.$ from 6 pups], Trkb ${ }^{\text {Gad1-KO }} 0.004 \pm 0.001 \mathrm{~Hz}\left[\mathrm{n}=14\right.$ from 3 pups], *** $\mathrm{p}<0.0001$ ); area (control $0.024 \pm 0.003 \mathrm{mVs}{ }^{-1}, T_{r k b}{ }^{G a d 1-K O}$ $\left.0.015 \pm 0.003 \mathrm{mVs}^{-1}{ }^{\star}{ }^{*} \mathrm{p}=0.03\right)$.

(C) Spontaneous and miniature GPSCs recorded from CA3 principal cells in hippocampal slices from P3 to P9 in Trkb Gad1-WT and Trkb Gad1-KO mice.

(D) Summary plots showing the mean frequency and amplitude of sGPSCs obtained from Trkb Gad1-WT ( $\mathrm{n}=15$ from 3 pups) and Trkb Gad1-KO ( $\mathrm{n}=18$ from 6 pups) mice, respectively (sGPCSs frequency: Trkb Gad1-WT $1.97 \pm 0.2 \mathrm{~Hz}$, Trkb ${ }^{\text {Gad1-KO }} 2.74 \pm 0.2 \mathrm{~Hz}$, ${ }^{\star} \mathrm{p}=0.03$; sGPCSs amplitude: Trkb Gad1-WT $50.3 \pm 4$ pA, Trkb Gad1-KO $64 \pm 6 \mathrm{pA}, \mathrm{p}=0.09)$.

(E) Cumulative distributions of interevent interval (IEI) of sGPSCs and mGPSCs for cells shown in (C).

(F) Summary plots showing the mean frequency and amplitude of mGPSCs obtained from Trkb Gad1-WT ( $\mathrm{n}=16$ from 5 pups) and Trkb Gad1-KO ( $\mathrm{n}=15$ from 4 pups) mice, respectively (mGPSCs frequency: Trkb Gad1-WT $0.97 \pm 0.1 \mathrm{~Hz}$, Trkb Gad1-KO $1.39 \pm 0.2 \mathrm{~Hz}$, *p $=0.04$; mGPSCs amplitude: Trkb Gad1-WT $45.5 \pm$ $\left.2.2 \mathrm{pA}, \mathrm{Trkb}^{\mathrm{Gad1}-\mathrm{KO}} 39.5 \pm 1.7 \mathrm{pA}, \mathrm{p}=0.07\right)$. Values are mean $\pm \mathrm{SEM}, \mathrm{p}$ statistic from unpaired Student's $\mathrm{t}$ tests.

(G-L) Increased number of GABAergic synapses in the CA3 region of Trkb Gad1-KO mice. Number of synapses in the CA3 hippocampal region/100 $\mu m^{2}$ at $P 5$,

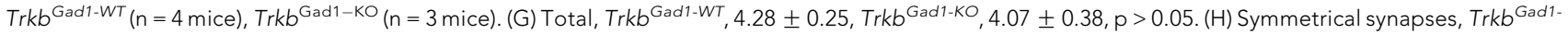
WT $, 1.40 \pm 0.09, T_{r k b}{ }^{G a d 1-K O}, 2.06 \pm 0.08, p=0.003$. (I) Asymmetrical synapses, control, $2.88 \pm 0.25, T r k b$ Gad1-KO $, 2.01 \pm 0.32, p=0.04$. (J and L)

Representative electron micrographs of single symmetrical synapses (white arrows) from Trkb Gad1-WT (J) and Trkb Gad1-KO (K) mice at P5. (L) Representative electron micrograph showing one asymmetrical synapse (black arrow) and two neighboring symmetrical synapses (white arrows) in the CA3 region of a Trkb Gad1-KO mouse at P5. Note the presence of a clear postsynaptic density at the asymmetric synapse that is lacking from the symmetric synapses. Values are mean \pm SEM, p statistic from unpaired one-tailed Student's $t$ tests. Scale bars: $0.5 \mu \mathrm{m}$ in $(\mathrm{J}$ and $\mathrm{K})$ and $500 \mu \mathrm{m}$ in $(\mathrm{L})$.

( $\mathrm{M}$ and N) No significant changes in the probability of GABA release at MF-CA3 synapses of Trkb Gad1-KO mice. (M) Representative examples of synaptic currents evoked in CA3 principal cells by pair stimulation of MF (50-ms interval). (N) Summary plots showing the mean paired-pulse ratio (PPR) values obtained in Trkb Gad1-WT $(n=7$ from 5 pups), $1.91 \pm 0.3$, and in Trkb Gad1-KO mice ( $n=10$ from 9 pups), $1.34 \pm 0.1 ; p=0.1$

See also Figures S3 and S4.

the primary determinant of the enhanced frequency of spontaneous and miniature GPSCs and are supportive of a presynaptic role of DGCs/TrkB signaling in the establishment of early synchronized activity and a proper excitatory/inhibitory balance within the CA3 hippocampal area. 


\begin{abstract}
Altered Chloride Homeostasis and the Polarity of GABA Action in Targeted Neurons
The enhanced GABAergic drive to CA3 principal cells may contribute to disrupting coherent network oscillations via a shunting inhibition caused by the premature shift of GABA action from the depolarizing to the hyperpolarizing direction. Immediately after birth, MFs release GABA that exerts a depolarizing and excitatory action on their targets (Sivakumaran et al., 2009). This action depends on the delayed temporal expression of the $\mathrm{Cl}^{-}$exporter $\mathrm{KCC} 2$ compared with an initial increased expression of the cation $/ \mathrm{Cl}^{-}$ importer NKCC1 (Blaesse et al., 2009). Therefore, to assess whether Trkb deletion from immature DGCs affects chloride homeostasis, thus altering the polarity of GABA action, we first measured the protein expression levels of NKCC1 and KCC2 by western blot (WB) from P7 hippocampal lysates. We found a significant decrease of NKCC1 in Trkb ${ }^{\text {Gad1-KO }}$ mice compared with controls (Figure 4A). This decrease was still apparent at P50 (Figure S5A). We detected no changes in KCC2 levels of monomeric or oligomeric forms between the two genotypes at P7 (Figure 4B), or at a later age (P50) (Figures S5B and S5C). Next, to visualize, localize, and quantify more precisely the amount of NKCC1 in cells of the CA3 hippocampal region we used single-molecule fluorescence in situ hybridization (smFISH) for Nkcc1 mRNA detection and quantification at P7. As shown in Figures $4 \mathrm{C}-4 \mathrm{G}$, the number of single mRNA molecules per cell detected in Trkb Gad1-KO mice was significantly diminished compared with controls, confirming the WB results.
\end{abstract}

We then tested whether the reduced expression of Nkcc1 alters the direction of GABA action at immature MFCA3 synapses. First, we measured the equilibrium potential of MF-evoked GPSCs ( $E_{\text {GPSC }}$ ) in CA3 principal cells using gramicidin perforated-patch recordings to prevent changes in intracellular chloride concentration $\left[\mathrm{Cl}^{-}\right]_{\mathrm{i}}$. We found that in Trkb ${ }^{G a d 1-K O}$ mice, compared with controls, the equilibrium potential of GABAergic postsynaptic currents $\left(E_{G P C s}\right)$ evoked by MF stimulation was shifted toward more hyperpolarized potentials (Figures $4 \mathrm{H}-4 \mathrm{~K})$. It is worth noting that whereas in control animals, seven of eight neurons exhibited $\mathrm{E}_{\mathrm{GABA}}$ values at least $15 \mathrm{mV}$ positive with respect to their resting membrane potentials (RMP), in Trkb ${ }^{\text {Gad1-KO }}$ mice only three of eleven cells exhibited similar values. In contrast, the remaining cells showed only small differences of few millivolts around their RMPs. The RMP values, estimated at the end of the experiments by breaking the membrane, were used to measure the driving force for MF-mediated GPSCs $\left(\Delta F_{G A B A}\right)$, being $\Delta F_{G A B A}=E_{G P C s}-R M P$. These values were significantly different between controls and Trkb Gad1-KO mice (Figure 4K). Altogether these data suggest that the reduced expression of $N k c c 1$ in Trkb ${ }^{G a d 1-K O}$ mice is sufficient to shift $E_{G A B A}$ toward more hyperpolarized values, close to their RMPs, thus exerting a shunting inhibition at the network level.

To further assess whether altered GABAergic signaling may account for network dysfunction in Trkb Gad1-KO immature hippocampus (P3-P9), we used cell-attached recording and examined the effects of isoguvacine, a specific $G_{A B A}$ agonist, on the spontaneous firing of CA3 principal cells. As expected, isoguvacine, applied by pressure from a pipette positioned close to recorded neurons, increased the firing rate of CA3 pyramidal cells in control animals (Khazipov et al., 2004), but it consistently reduced it in Trkb Gad1$K O$ mice (Figures $4 \mathrm{~L}-4 \mathrm{~N}$ ). In conclusion, these experiments demonstrate that reduced TrkB signaling in DGCs, early in postnatal development, leads to a reduced expression of the cation-chloride importer NKCC1. The lower $\left[\mathrm{Cl}^{-}\right]_{i}$ produces a premature shift of GABA from the depolarizing to the hyperpolarizing direction with consequent inhibitory effect at the network level.

\title{
Enhanced GABA -Mediated Tonic Inhibition in the Hippocampus of Trkb $^{\text {Gad1-KO }}$ Mice
}

The data reported in the previous section clearly show that GABA exerts an inhibitory effect on networkdriven GDPs via a shunting inhibition. This inhibitory action may be further boosted by a tonic $\mathrm{GABA}_{A}$-mediated conductance, following activation of extrasynaptic $\mathrm{GABA}_{A}$ receptors by spillover of GABA from adjacent synapses. Such conductance is known to be altered in several forms of neurodevelopmental disorders (Brickley and Mody, 2012; Cellot et al., 2016). To test this hypothesis, we measured the shift in the holding current induced by bath application of the GABA $A$ receptor channel blocker picrotoxin $(100 \mu \mathrm{M})$ in the presence of DNQX $(20 \mu \mathrm{M})$ and DL-APV $(100 \mu \mathrm{M})$. This caused a more pronounced shift in the holding current in Trkb Gad1-KO compared with control mice (Figures $4 \mathrm{O}$ and P). These results suggest that indeed an increased $\mathrm{GABA}_{A}$-mediated tonic conductance activated by GABA released from GABAergic interneurons may contribute to altering GDPs.

\section{The Premature Shift of GABA from the Depolarizing to the Hyperpolarizing Direction Affects the Maturation of CA3 Principal Cells and Impairs LTP at MF-CA3 Synapses}

To investigate whether changes in network activity and GABAergic signaling following Trkb deletion from the hippocampal immature DGCs affect CA3 principal cell maturation, we examined the morphology of 


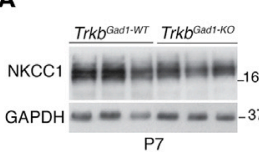

B

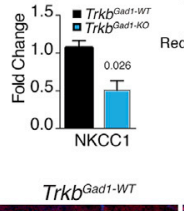

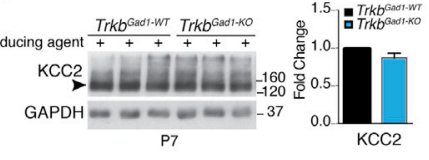

Trkb $b^{\text {Gad1-WT }}$

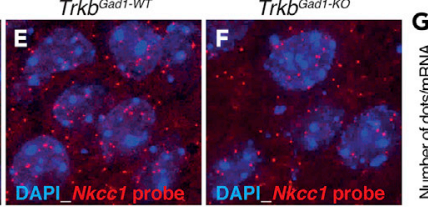

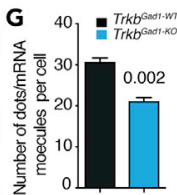

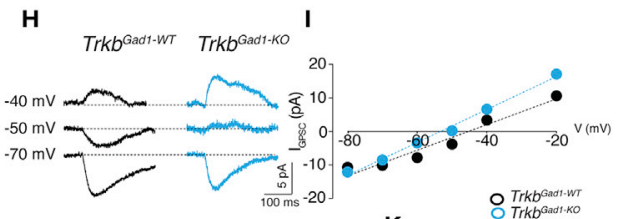

J TrkbGadt-WT
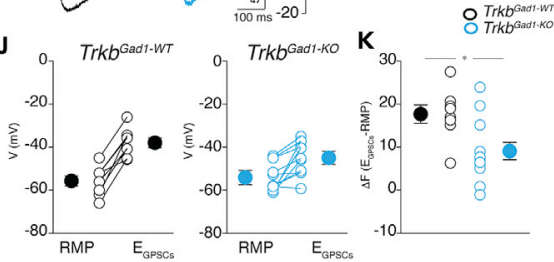

L

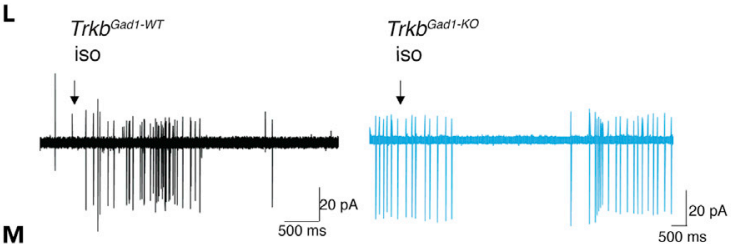

M $500 \mathrm{~ms}$

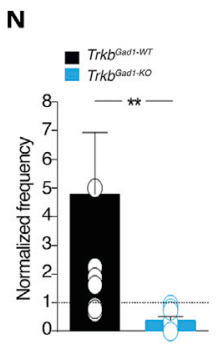

o

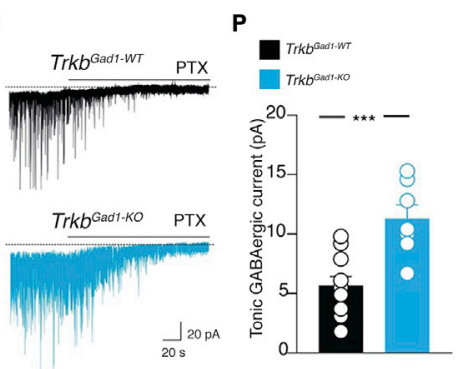

Figure 4. Reduced Expression of Nkcc1 and Altered Direction of GABA Action at Immature MF-CA3 Synapses in Trkb ${ }^{\text {Gad1-KO }}$ Mice (A and B) Representative western blots from P7 hippocampal lysates and relative quantification of NKCC1 protein levels (P7, Trkb Gad1-WT [n $=6$ ], $1.065 \pm$

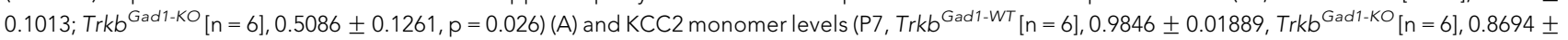
$0.06086, p=0.1)(B)$. GAPDH, loading control.

(C-G) Single-molecule fluorescence in situ hybridization (smFISH) was used to detect and count individual Nkcc1 RNA molecules in single cells of the CA3 region at P7. (C) Representative image of a P7 hippocampal section stained with DAPI. Highlighted are three random fields imaged in the CA3 region for quantification of single-molecule RNA (more details in Transparent Methods). (D-F) Representative images from the CA3 regions of control and mutant mice highlighting the single cells by DAPI nuclear staining. The red spots corresponding to single mRNA molecules derived from the transcription of Nkcc1 are detected with the Quasar570 fluorophore-labeled oligonucleotide probe library in single cells of the CA3 hippocampal region (probe details in Table S4) (E

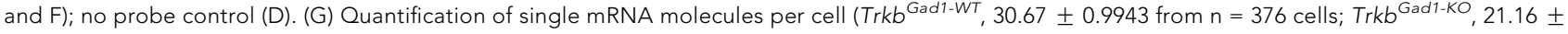
0.8630 from $n=396$ cells, $p=0.002 ; n=3$ P7 pups each genotype). DGL, dentate granule layer. Scale bars: $250 \mu \mathrm{m}$ in (C) and $50 \mu \mathrm{m}$ in (D-F).

$(\mathrm{H}-\mathrm{K})$ Reduced driving force for GABA-mediated postsynaptic currents (GPSCs) at MF-CA3 synapses in Trkb Gad1-KO mice. (H) Representative traces of GPSCs evoked at three different holding potentials in CA3 principal cells by MF stimulation (gramicidin-perforated patches) in Trkb ${ }^{\text {Gad1-WT }}$ and Trkb Gad1-KO mice. (I) Amplitudes of GPSCs ( $\mathrm{I}_{\text {GPSC }}$ ) shown in (H) are plotted against holding potentials $(\mathrm{V})$. (J) Individual RMPs and $E_{\mathrm{GPSCs}}$ values in CA3 principal cells from control ( $n=8$ from 5 pups) and Trkb Gad1-KO ( $n=11$ from 7 pups). Larger symbols on the left and right refer to mean \pm SEM values (RMPs, Trkb ${ }^{\text {Gad1-WT }},-55.6 \pm$

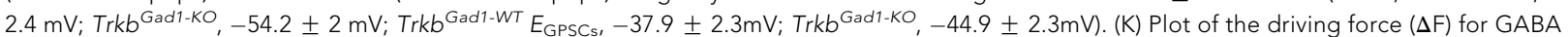
$\left(\Delta F=E_{\mathrm{GPSCs}}-R M P\right)$ in individual experiments from Trkb Gad1-WT and Trkb Gad1-KO mice. Larger symbols are mean $\pm \mathrm{SEM}$ values. $\Delta \mathrm{F}=17.7 \pm 2.1 \mathrm{mV}$ in Trkb Gad1WT and $9.1 \pm 2.2 \mathrm{mV}$ in Trkb ${ }^{\text {Gad1-KO }}$ mice; ${ }^{*} \mathrm{p}=0.03$, Wilcoxon test.

(L-N) Altered GABAergic signaling accounts for network dysfunction in Trkb Gad1-KO immature hippocampus. Effects of isoguvacine on spontaneous firing of CA3 principal cells in Trkb ${ }^{G a d 1-W T}$ and Trkb ${ }^{G a d 1-K O}$ mice. (L) Two representative examples of changes in spontaneous firing induced by pressure application of isoguvacine (100 $\mu \mathrm{M}$ for $1 \mathrm{~s}$; arrows) to CA3 principal cells (recorded in cell-attached) in Trkb Gad1-WT (black) and Trkb Gad1-KO mice (cyan). (M) Interspike interval histograms (bin: $10 \mathrm{~ms}$ ) for cells shown in (L). (N) Summary plot showing isoguvacine-induced changes in spike frequency (30 s after drug application) normalized to baseline values (30 s before drug application); Trkb ${ }^{\text {Gad1-WT }}, 4.78 \pm 2.1$ ( $n=9$ from 4 pups), Trkb ${ }^{\text {Gad1-KO }}, 0.39 \pm 0.1$ ( $n=8$ from 5 pups); ${ }^{\star \star} p=$ 0.025. Values are mean \pm SEM, p statistic from unpaired Student's t test.

$\left(O\right.$ and $P$ ) Increased GABA $A_{A}$-mediated tonic conductance in Trkb ${ }^{G a d 1-K O}$ mice. (O) Representative traces of spontaneous $G_{A B A}$-mediated synaptic currents (sGPSCs) recorded from CA3 principal cells before and during application of picrotoxin (PTX, $100 \mu \mathrm{M}$ bars above the traces) in hippocampal slices obtained from control $(n=11)$ and Trkb Gad1-KO mice $(n=7)$. Note the upward shift of the baseline current and disappearance of sGPSCs after application of PTX $(100 \mu \mathrm{M})$ in the presence of DNQX $(20 \mu \mathrm{M})$ and DL-APV $(100 \mu \mathrm{M})$. (P) Each column represents the mean tonic GABA ${ }^{-m e d i a t e d ~ c o n d u c t a n c e ~ m e a s u r e d ~ i n ~}$ controls, $5.7 \pm 0.75 \mathrm{pA}(n=11)$ and Trkb ${ }^{\text {Gad1-KO }}$ mice, $11.3 \pm 1.2 \mathrm{pA}(n=7) .{ }^{\star \star *} \mathrm{p}=0.0008$, Mann-Whitney test.

See also Figures S5 and Table S4.

CA3 principal cells at young adult age (2M) by Golgi staining (Figure 5A). Trkb Gad1-KO mice showed a significant reduction in proximal dendritic segment length compared with controls (Figure 5B). The number of dendritic intersections was also significantly reduced in $T r k b^{G a d 1-K O}$ mice only on apical dendrites located 
A
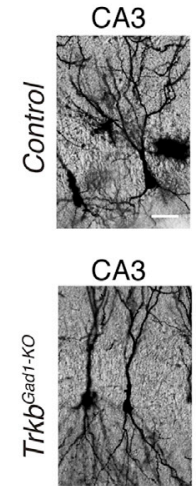
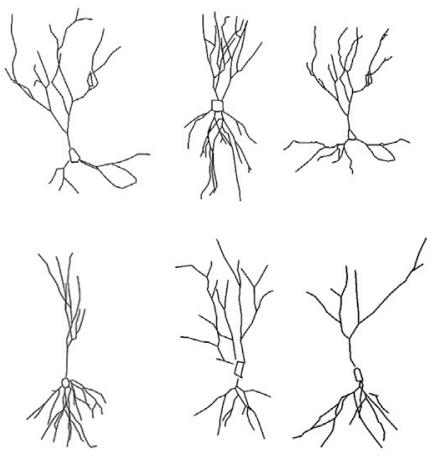

B

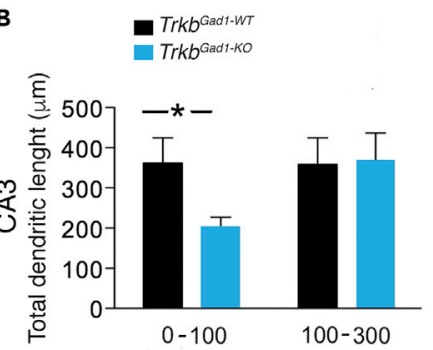

Distance from soma $(\mu \mathrm{m})$

\section{c}

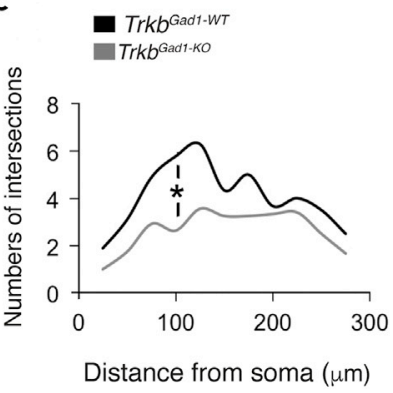

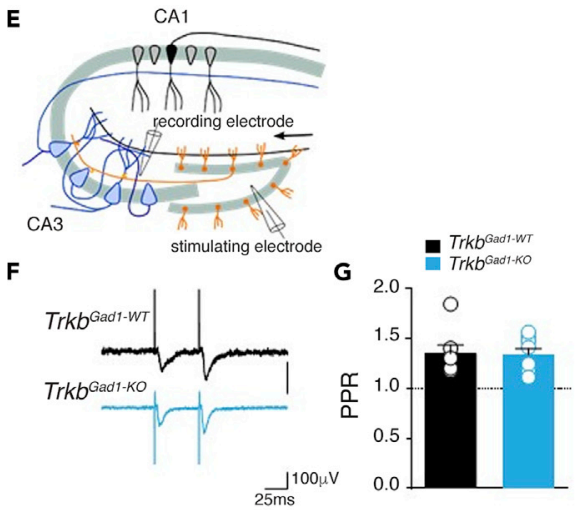

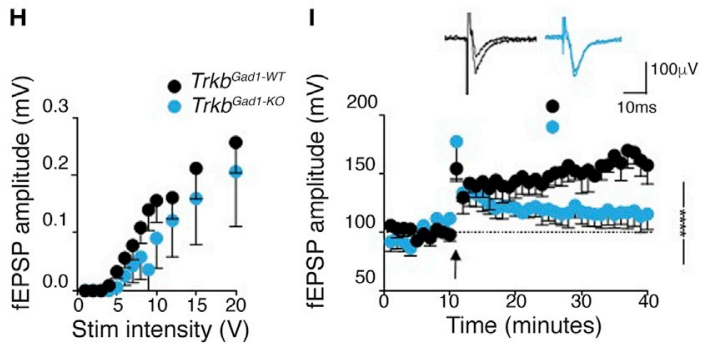

D

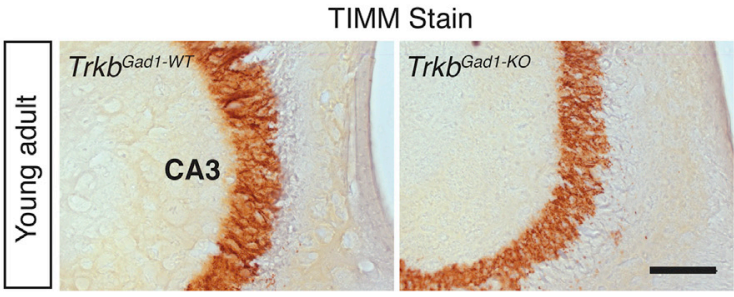

Figure 5. Morphological Alterations of CA3 Principal Cells and Impaired Hippocampal LTP at MF-CA3 Synapses in Adult Trkb Gad1-KO Mice (A) Representative images of Golgi-stained (left) and neurolucida tracings (right) of CA3 principal cells from young adult control (Trkb Gad1-WT) and Trkb Gad1KO mice.

(B) Quantitative analysis of dendritic length of CA3 principal neurons in Trkb Gad1-WT and in Trkb ${ }^{\text {Gad1-KO }}$ mice. Total dendritic length, proximal 1-100 $\mu m$ from soma (Trkb Gad1-WT, $362.32 \pm 65.51$; Trkb ${ }^{\text {Gad1-KO }}, 203.75 \pm 23.78$, Student's t test: $t_{(19)}=2.59$; $\left.{ }^{*} \mathrm{p}=0.017\right)$, and distal 100-300 $\mu$ m from soma (Trkb ${ }^{\text {Gad1-WT }}$, $358.13 \pm 94.33 ;$ Trkb $^{\text {Gad1-KO }}, 369.82 \pm 107.61$, Student's t test: $\left.t_{(18)}=0.26 ; p=0.79\right)$.

(C) Sholl analysis of principal neurons in Trkb Gad1-WT and Trkb Gad1-KO mice. Statistically significant differences across the dendritic trees between genotypes

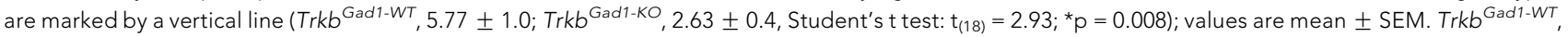
$\mathrm{n}=9$ neurons from 4 mice; Trkb ${ }^{\text {Gad1-KO}}, \mathrm{n}=12$ neurons from 3 mice.

(D) Representative images of Timm stain per genotype showing similar MF pattern in the CA3 target region of $2 \mathrm{M}$-old mice ( $\mathrm{n}=3$ each group).

(E) LTP at MF-CA3 synapses. Cartoon illustrating the experimental paradigm.

(F) Sample traces of paired field ( $f$ ) EPSPs (50-ms interval) evoked in CA3 stratum radiatum (sr) upon stimulation of MF in stratum lucidum ( $\mathrm{s}$ ) in the presence of DL-AP5 (100 $\mu \mathrm{M})$.

(G) Each column represents the mean value of the paired-pulse ratio (PPR, 50-ms interval) obtained from Trkb Gad1-WT $(1.35 \% \pm 9 \%, \mathrm{n}=7 \mathrm{slices}$ from $4 \mathrm{mice}$ ) and from Trkb ${ }^{\text {Gad1-KO }}$ mice (1.33\% $\pm 6 \%, \mathrm{n}=8$ slices from 3 mice), $\mathrm{p}=0.92$.

(H) Input-output relationship of fEPSP responses evoked in CA3 stratum radiatum by MFs stimulation in the stratum lucidum of Trkb Gad1-WT $(n=9$ slices from 5 mice) and Trkb Gad1-KO mice ( $n=5$ slices from 5 mice), Kolmogorov-Smirnov test, $p=0.998$. Each point represents the mean \pm SEM.

(I) The amplitudes of MF-fEPSPs recorded from Trkb ${ }^{\text {Gad1-WT }}(\mathrm{n}=4$ slices from 3 mice) and Trkb Gad1-KO mice ( $\mathrm{n}=5$ slices from 4 mice) before and after thetabursts stimulation (arrow, 10 trains $200 \mathrm{~Hz}, 50 \mathrm{~ms}$ each, repeated twice at $0.1 \mathrm{~Hz}$ ) of MF was normalized to baseline values and plotted against time. Mean amplitude values of fEPSPs evoked 10 min before and 30 min after LTP induction (30 min, fEPSPs amplitude, Trkb Gad1-WT, $160 \% \pm 2.1 \%, n=4$ slices from 3 mice; Trkb ${ }^{\text {Gad1-KO }}, 116.1 \% \pm 0.52 \%, \mathrm{n}=5$ slices from 4 mice, $\left.\mathrm{p}<0.001\right)$. The insets above the graph show two superimposed average traces of fEPSPs obtained from Trkb Gad1-WT and Trkb ${ }^{\text {Gad1-KO }}$ mice before and $30 \mathrm{~min}$ after LTP induction.

Scale bars: $500 \mu \mathrm{m}$ in (A) and $100 \mu \mathrm{m}$ in (D). 
within $100 \mu \mathrm{m}$ from the soma. No differences between the two genotypes were detected in more distal dendrites (>100 $\mu \mathrm{m}$ from the soma) (Figure 5 C), or in basal dendrites (ANOVA, $F_{6,114}=0.283 ; p>0.05$ ). However, the observed morphological alterations of the CA3 principal cells were not due to lack of MF terminals, making synaptic contact onto CA3 principal cells. The use of Timm stain, which detects the Zn2+-containing MF terminals, revealed similar MF bundles between Trkb Gad1-KO mice and controls (Figure $5 \mathrm{D}$ ), confirming both the tdTomato reporter signal (Figures $1 \mathrm{~A}-1 \mathrm{~F}$ ) and the Calbindin immunostaining (Figures 1G, 1I, 1K, and 1M).

Hence, we tested the hypothesis that morphological alterations in CA3 principal cells due to the absence of presynaptic TrkB signaling may alter the development of functional neuronal circuits and storage of information leading to altered long-term potentiation (LTP) and behavioral impairment in adult animals. MFCA3 synapses are known to undergo robust NMDA-independent LTP following theta-burst activation of the MF pathway in stratum lucidum (Nicoll and Schmitz, 2005). Therefore, field EPSPs (fEPSPs) evoked by stimulation of MF in stratum lucidum were routinely recorded in hippocampal slices (from 2- to 3M-old animals) in the presence of DL-AP5 $(100 \mu \mathrm{M})$, a selective NMDA receptor antagonist. In basal conditions (0.1- $\mathrm{Hz}$ stimulation), we detected no changes in synaptic transmission, cell excitability, or short-term plasticity between the two genotypes (Figures 5E-5H). The PPR, a typical presynaptic form of short-term plasticity, was similar between Trkb ${ }^{G a d 1-K O}$ mice and control littermates (Figures 5F and 5G). However, whereas theta-burst stimulation induced persistent changes in synaptic efficacy in control animals, the same stimulation induced only a transient increase in synaptic efficacy in Trkb Gad1-KO mice that further decreased over time to baseline levels. There was a significant difference in LTP magnitude between the two genotypes (Figure 5I).

\section{In Vivo, LTP Is Depressed at the Schaffer Collateral-CA1 Synapses in Trkb ${ }^{\text {Gad1-KO }}$ Mice}

The CA3 pyramidal neurons project mainly to the CA1 pyramidal neurons via Shaffer collaterals. As presynaptic deletion of Trkb affected synaptic strength at MF-CA3 synapses, we asked if consequently synaptic plasticity was also altered at Schaffer collateral-CA1 synapses. Therefore, we investigated synaptic plasticity at Schaffer collateral-CA1 synapses where LTP is known to last for hours to days in alert-behaving adult animals. No changes in basal synaptic transmission and short-term plasticity (paired-pulse facilitation) were observed between the two genotypes and additional control groups (BAC-Gad1-Cre ${ }^{\text {tg/+ }}$ animals compared with wild-type [WT] littermates) (Figures S6A and S6B, and Table S1). However, LTP evoked in the control littermates (Trkb Gad1-WT ) was significantly larger and longer lasting (up to $48 \mathrm{~h}$ ) compared with that evoked in the mutant Trkb Gad1-KO group (Figures 6A-6C, and Table S2). Also, the BAC-Gad1$\mathrm{Cre}^{\text {tg/+ }}$ animals compared with WT littermates presented similar LTP lasting for $48 \mathrm{~h}$ (Figures S6C-S6E, and Table S3). These data suggest that alert-behaving Trkb Gad1-KO animals are unable to present a significant increase in fEPSP slopes evoked at the CA3-CA1 synapses following the high-frequency stimulation session applied to the Schaffer collaterals/commissural pathway. Therefore, selective Trkb deletion in immature DGCs severely alters the establishment of hippocampal circuitry and storage of information downstream of DGCs, leading to synaptic plasticity impairment in adulthood.

\section{The Behavioral Correlate of Neural Circuits Downstream of MF-CA3 Synapses Is Selectively Affected in Trkb Gad1-KO Mice}

As deletion of Trkb in immature DGCs affected synaptic strength at MF-CA3 and Schaffer collateral-CA1 synapses, we asked whether these alterations would specifically affect the processing of information that depends on intact hippocampal regions. Therefore, we tested Trkb Gad1-KO and control mice in two behavioral tasks, the object-context recognition (OCR) and the contextual fear conditioning (FC). In rats, the OCR has been shown to critically rely on the lateral entorhinal cortex (LEC), which processes the contextual features of an event (Wilson et al., 2013). Mice were first tested in an open field where both mutants and controls showed habituation to the arena and normal anxiety behavior (Figures S7A-S7C). Then, we tested mice in the OCR task (experimental design explained in Figure S7D). Both genotypes showed no difference in the discrimination ratio (Figure 7A). Similarly, the total object exploration time during the acquisition phase and the test session was not significantly different between genotypes (Figures 7B and 7C), suggesting that the LEC is intact in these mutants. We then tested mice for an associative learning task such as contextual FC. In this form of learning an aversive stimulus (electrical shock) is associated with a specific neutral context, or a neutral stimulus (tone). Trkb Gad1-KO mice showed impaired neutral context acquisition/recognition but normal response to a conditioned neutral stimulus compared with control littermates or the BAC-Gad1-Cre (Figures 7D-7F and 7G-7I). Because contextual FC involves all three hippocampal 
A
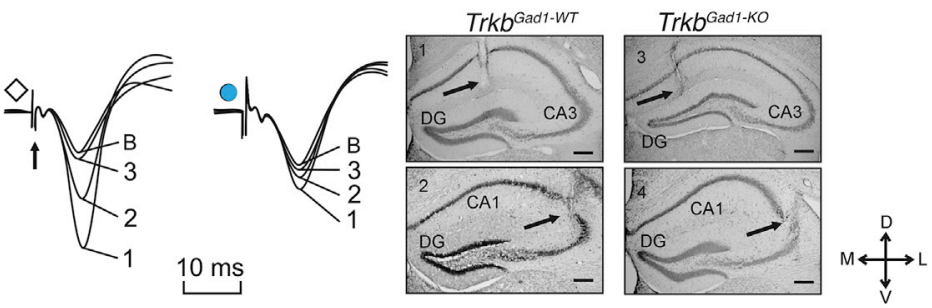

B

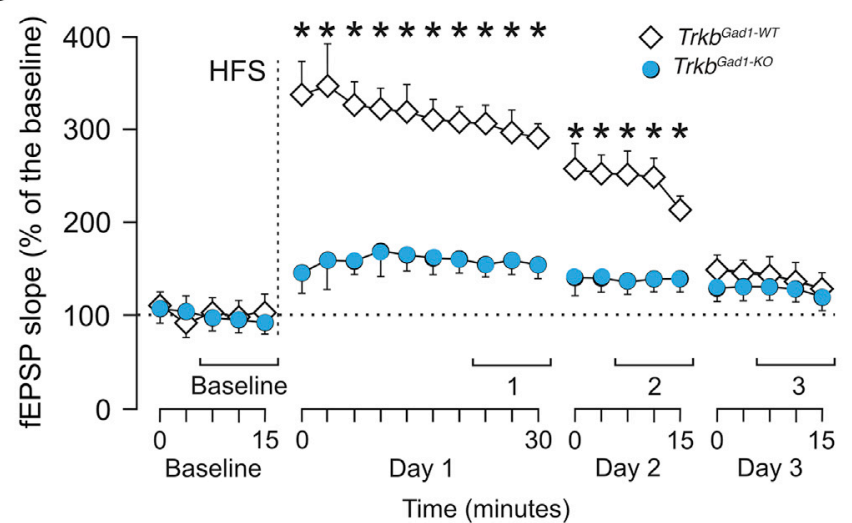

C

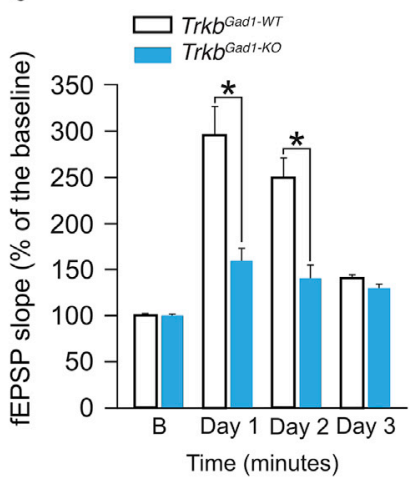

Figure 6. In Vivo Recordings of LTP in the CA1 Hippocampal Area Following Electrical Stimulation of Schaffer Collaterals

(A) Representative example fEPSP recordings collected from selected animals of each experimental group at the times indicated in (B). In all cases, the smaller fEPSP was collected before the high-frequency stimulation (HFS) (baseline), whereas the larger one was collected 24-30 min after the HFS. On the right, images illustrating the location of recording (1 and 3 ) and stimulating (2 and 4) sites (arrows) are shown.

(B) Time course of changes in fEPSP slopes (mean \pm SEM) following HFS stimulation of Schaffer collaterals. Baseline recordings were collected for $15 \mathrm{~min}$ at a rate of $3 \mathrm{pulse} / \mathrm{min}$. As indicated in the Methods, we then evoked LTP by HFS protocol consisting of five trains $(200 \mathrm{~Hz}, 100 \mathrm{~ms})$ of pulses at a rate of $1 / \mathrm{s}$. This protocol was presented six times in total, at intervals of $1 \mathrm{~min}$. After HFS, we presented the same single stimulus used to generate baseline records at the initial rate (3/ $\mathrm{min}$ ) for another $30 \mathrm{~min}$. Additional sets of recordings (15 min each) were obtained 24 and $48 \mathrm{~h}$ after HFS. fEPSPs are given as a percentage of baseline (100\%) values. Following the HFS session, fEPSPs were recorded for $30 \mathrm{~min}$ on day 1 and for 15 min on the following days. *Significant differences between Trkb ${ }^{G a d 1-W T}$ and Trkb ${ }^{\text {Gad1-KO }}$ group $\left(F_{(24,336)}=36.839 ; p<\right.$ $0.001 ; n=8$ animals per group. Two-way repeated measures ANOVA, one factor repetition).

(C) fEPSP data (mean \pm SEM) included in each histogram (baseline, days 1,2, and 3) were collected for the time intervals indicated in $(B) .{ }^{*} F_{(3,118)}=93.67 ; p<0.001 ; n=8$ animals per group. Two-way repeated measures ANOVA, one factor repetition].

Scale bars: $250 \mu \mathrm{m}$ in (A). Abbreviations: D, L, M, and V, dorsal, lateral, medial, ventral, respectively; DG, dentate gyrus. See also Figure S6 and Tables S1, S2, and S3.

areas, DG, CA3, and CA1, to integrate impulses from the amygdala (Lee and Kesner, 2004), a structure in the brain needed for the expression of fear, these results further support that presynaptic depletion of TrkB signaling from immature DGCs affects specifically neuronal circuits located within the hippocampus proper downstream of MF-CA3 synapses.

\section{DISCUSSION}

This study uncovers the requirement of TrkB signaling for the establishment of excitatory/inhibitory homeostasis at MFs-CA3 synapses, critical for the development of functional hippocampal circuits. DGCs, via MFs, transfer incoming cortical information in sparse and specific hippocampal signals, which are essential for memory formation. The selective deletion of Trkb from immature DGCs early in postnatal development affects the flow of information to the hippocampus proper, thus preventing the establishment of proper synaptic connectivity in targeted neurons. This leads to the disruption of GDPs, a primordial form of synchrony between neurons known to play a critical role in neuronal growth and synaptogenesis (Ben-Ari et al., 2012). Several factors may contribute to altering GDPs in Trkb Gad1-KO mice, including the early shift of GABA from the depolarizing to the hyperpolarizing direction with a consequent increase in 
A

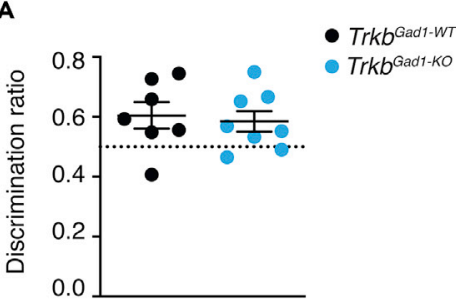

B

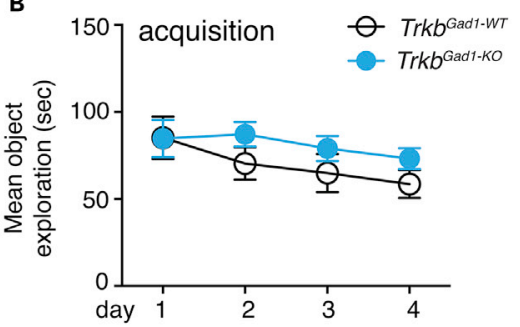

D

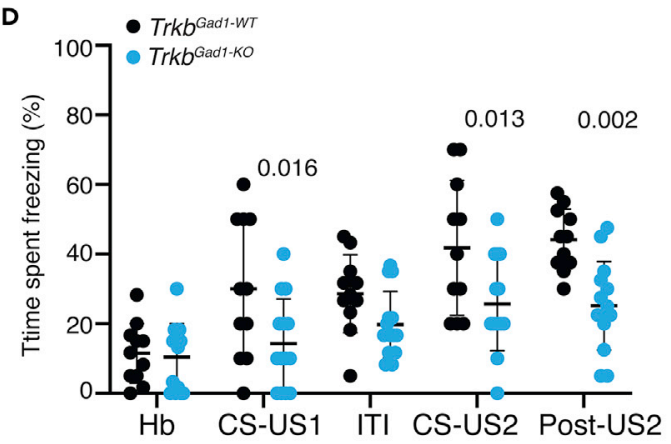

G

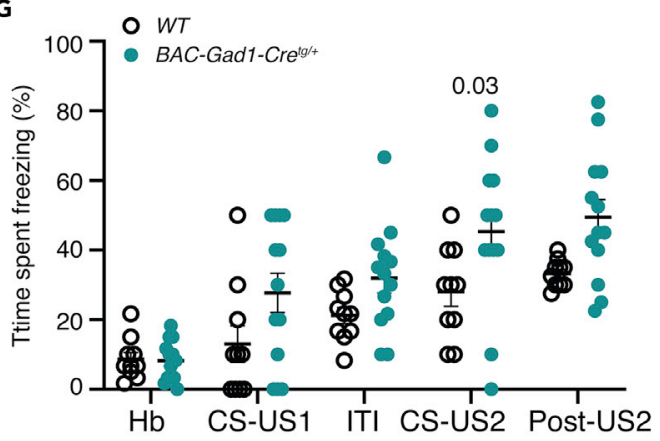

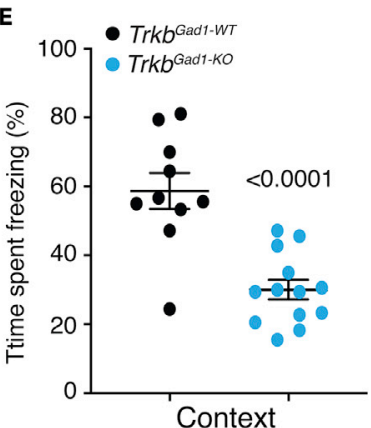

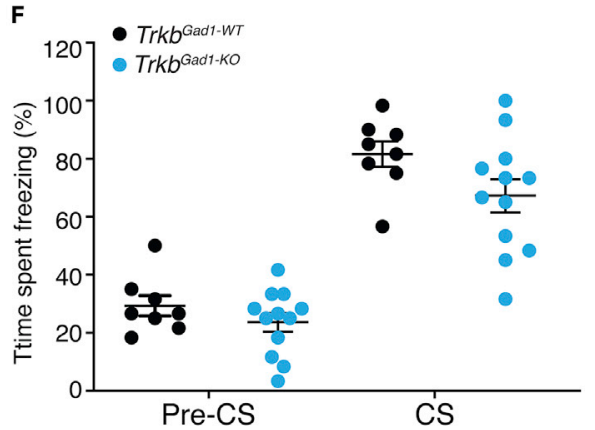

H

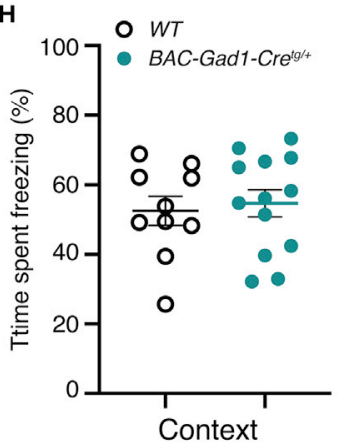

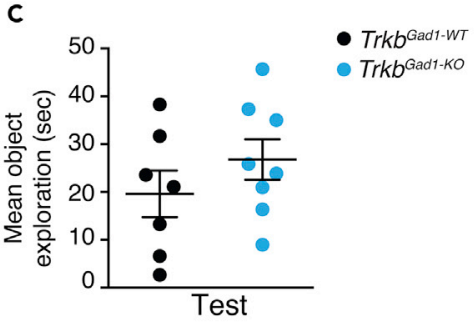

I

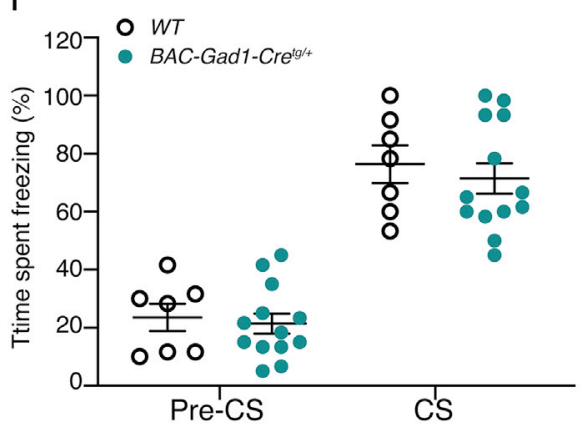

Figure 7. Normal Context-Object Recognition but Affected Neutral Context Recognition in Trkb ${ }^{\text {Gad1-KO }}$ Mice

(A-C) Context-object recognition test. (A) Mean discrimination ratio was calculated as a ratio of the time spent exploring an object not previously paired with the context over the total time spent observing both paired and unpaired objects. No difference was found between genotypes (Trkb Gad1-WT $[\mathrm{n}=7$ ] $0.61 \pm$ 0.04; Trkb ${ }^{\text {Gad1-KO }}[\mathrm{n}=8] 0.59 \pm 0.03$, mean \pm SEM, unpaired Student's t test, $\left.\mathrm{p}=0.7195\right)$. (B) Mean total object exploration time during the 4-day acquisition phase was similar between Trkb mutant and control mice and decreased significantly over time (two-way ANOVA repeated measures: $F_{(3,39)}=3.276, p=$ 0.031, main effect of time). (C) The mean total object exploration time analyzed during the test session was also not significantly different between genotypes $\left(\right.$ Trkb ${ }^{\text {Gad1-WT }}, 19.61 \pm 4.9 ;$ Trkb $^{\text {Gad1-KO }}, 26.78 \pm$ 4.23; mean \pm SEM; unpaired Student's t test: $\left.t_{(13)}=1.112, p=0.2861\right)$.

(D-F) Fear conditioning test. (D) Acquisition phase. Two-way ANOVA repeated measures (Trkb Gad1-WT, $\mathrm{n}=10 ;$ Trkb Gad1-KO, $\mathrm{n}=13$ ) revealed a main effect of genotype in freezing behavior $\left(F_{(1,23)}=10.56, p=0.0035\right)$, a main effect of test stage $\left(F_{(4,92)}=22.1, p<0.0001\right)$, and an interaction between genotype and test stage $\left(F_{(4,92)}=3.072, p=0.0201\right)$. To explore the observed interaction, Sidak's multiple corrected comparisons showed a statistically significant difference between genotypes in the percentage time spent freezing during the CS-US1 (Trkb Gad1-WT, 30.00\% \pm 6.03\%; Trkb Gad1-KO, $14.29 \% \pm 3.43 \%$; mean \pm SEM, $\mathrm{p}=0.0166)$, during the CS-US2 (Trkb ${ }^{\text {Gad1-WT }}, 41.82 \% \pm 5.85 \%$; Trkb $\left.{ }^{\text {Gad1-KO }}, 25.71 \% \pm 3.59 ; \mathrm{p}=0.013\right)$, and in the post-US2 (Trkb Gad1-WT, $44.09 \% \pm 2.68 \%$; Trkb Gad1-KO $25.18 \% \pm 3.39 \% ; p=0.002)$. (E) Twenty-four hours after conditioning, mice were first tested for contextual fear conditioning. A statistically significant difference in the time spent freezing was found between control ( $n=10), 58.72 \% \pm 5.22 \%$, and Trkb Gad1-KO $(n=13)$ mice, 30.04\% $\pm 2.84 \%$; mean \pm SEM, unpaired Student's t test, $p<0.0001$, two-tailed. (F) Twenty-four hours later mice were tested for cued fear conditioning. Two-way ANOVA repeated measures (TrkbGad1-WT, $\mathrm{n}=8$; Trkb Gad1-KO, $\mathrm{n}=12$ ) revealed a main effect of test stage $\left(\mathrm{F}_{(1,18)}=92.3, \mathrm{p}<0.0001\right)$ and a main effect of genotype $\left(F_{(1,18)}=5.446, p=0.0314\right)$, but no interaction $\left(F_{(1,18)}=0.7558, p=0.3961\right)$. Sidak's multiple comparisons test showed no significant difference between control and mutant mice in freezing responses (\%) (Trkb Gad1-WT, $81.67 \% \pm 4.40 \%$; Trkb Gad1-KO $, 67.22 \% \pm 5.77 \%, p=0.07)$.

(G-I) Context and cued fear conditioning are unaffected in BAC-Gad1-Cre mice. (G) Percentage of time spent freezing during conditioning. Two-way ANOVA repeated measures (BAC-Gad1-Cre mice, $n=13 ; \mathrm{WT}$ control, $n=10)$ revealed a main effect of genotype in freezing behavior $\left(F_{(1,21)}=8.477, p=\right.$ $0.0083)$ and a main effect of the stage of the test $\left(F_{(4,84)}=24.43, p<0.0001\right)$, but no interaction between genotype and test stage $\left(F_{(4,84)}=1.865, p=0.1241\right)$. Sidak's multiple corrected comparisons showed a statistically significant difference between genotypes in the percentage of time spent freezing during the second tone-foot shock presentations (CS-US2, WT: $28.00 \% \pm 4.16 \%$, BAC-Gad1-Cre: $45.38 \% \pm 6.06 \%, p=0.0305$ ) compared with the other stages (CSUS1, WT 13.00\% \pm 5.17\%, BAC-Gad1-Cre: $27.69 \% \pm 5.68 \%, p=0.09$; post-US2, WT: $33.25 \% \pm 1.24 \%$, BAC-Gad1-Cre: $49.42 \% \pm 5.15 \%, p=0.052) .(H)$ 
Figure 7. Continued

Twenty-four hours after conditioning, mice were first tested for contextual fear conditioning, and no difference was found in freezing responses (\%) between WT $(n=10)$ and BAC-Gad1-Cre mice $(n=13)$ (\% of time spent freezing, WT: 52.53\% \pm 4.17\%, BAC-Gad1-Cre: 54.72\% \pm 3.92\%; unpaired Student's t test, $p=0.697$, two tailed). (I) Cued fear conditioning was tested $24 \mathrm{~h}$ later. Two-way ANOVA repeated measures revealed a main effect of test stage $\left(F_{(1,18)}=266.3, p<0.0001\right)$, but no effect of genotype $\left(F_{(1,18)}=0.2818, p=0.6020\right)$. Freezing responses $(\%)(W T, n=7, B A C-G a d 1-C r e, n=13)(\%$ of time spent freezing, CS, WT: $76.43 \% \pm 6.48 \%, B A C-G a d 1-C r e: 71.54 \% \pm 5.25 \%, p=0.76$ ). Values are mean \pm SEM. ITI, intertrial interval; Hb, habituation; CS, conditioned stimulus; US, unconditioned stimulus.

See also Figures S7.

early phasic and tonic $\mathrm{GABA}_{A}$-mediated inhibition, resulting in a shunting action at the network level. These changes affect cellular morphology, maturation, and circuit formation downstream of the MFsCA3 synapses with permanent deficits in hippocampal synaptic plasticity and cognitive functions resembling a neurodevelopmental disorder condition.

Both BDNF and TrkB are expressed at high levels by granule cells and are essential regulators of granule cell morphology (Danzer et al., 2008) (Berg et al., 2019). Here, in particular, we have demonstrated that deletion of Trkb from immature DGCs early in postnatal development affects their integration and maturation into the DGC layer possibly due to delayed migration. This phenotype is supported by the accumulation of DCX+ immature granule cells in the SGZ of mutant compared with control mice at 3 weeks age followed by visibly less accumulated DCX+ cells in the SGZ and more tdTomato+ cells in the GCL at $2 \mathrm{M}$ age. Moreover, we have studied CREB activation to further support this hypothesis. CREB signaling plays a crucial role in adult hippocampal neurogenesis by regulating the development of newly generated immature DGCs downstream of GABA-mediated excitation (Jagasia et al., 2009). We show that similarly to adult neurogenesis, the peak of CREB activation at P21 in control mice was predominantly detected in the lower third of the DGL at the hilar border where newborn immature neurons reside. Such signal declined by $2 \mathrm{M}$ age, coinciding with reduced neurogenesis. In the absence of TrkB signaling, however, there was a reduced activation of CREB at P21 compared with controls followed by a decline at $2 \mathrm{M}$. However, CREB activation at $2 \mathrm{M}$ was apparently higher in mutants than in controls, reflecting the delayed migration of DCX+ cells still at this age. Finally, we also show that the morphological maturation of the newly formed DGCs is impaired. Thus, these results support that delayed migration accounts at least in part for the fewer tdTomato cells in the GCL.

BDNF, via TrkB signaling, plays a crucial role in the maturation of inhibition as demonstrated in visual cortical neurons and cultured hippocampal cells (Huang et al., 1999; Yamada et al., 2002). Therefore, it is not surprising that Trkb deletion in Trkb ${ }^{\text {Gad1-KO }}$ mice affects GDPs, generated in the immature hippocampus by the synergistic action of glutamate and GABA. The intracellular concentration of $\mathrm{Cl}^{-}$, which depends on the developmentally regulated expression of the cation-chloride importer and exporter NKCC1 and KCC2, respectively (Rivera et al., 1999), determines the strength and the direction of $\mathrm{GABA}_{A}$-mediated transmission. In immature neurons, increasing BDNF/TRKB signaling in vitro promotes the developmental upregulation of KCC2 expression (Aguado et al., 2003), whereas in this study we show that selective in vivo reduction of Trkb from immature DGCs leads to a hippocampal decrease of Nkcc1, in particular, in the CA3 principal cells shown by smFISH. No influence was detected on KCC2 expression. NKCC1 is known to promote network activity in the CA3 hippocampal region (Sipila et al., 2006) and the lack of Nkcc1 in knockout mice leads, like in our experiments, to GDP disruption (Pfeffer et al., 2009). However, the question is how depletion of BDNF-TrkB signaling from immature DGCs regulates the expression of the chloride importer, Nkcc1, in targeted CA3 principal cells. Although how exactly this occurs is still largely unknown and requires further investigation, we provide the following explanations. First, this depletion may affect the integration of excitatory inputs onto DGCs and their activity, as suggested by the reduced number of spines on apical dendrites of these cells. Changes in neuronal activity may disrupt chloride homeostasis known to be particularly sensitive to both activity-dependent processes and BDNF-regulated neuronal activity (Fiumelli and Woodin, 2007). Also, there is evidence that BDNF/TrkB signaling can act as a transsynaptic organizer of neuronal circuits. For example, in mice carrying conditional Trkb deletion induced by Synapsin1-Cre strain an increased number of filopodial extensions contacting GABAergic interneurons has been found, which provide feedforward inhibition to CA3 principal cells (Danzer et al., 2008). Consistent with these findings, in the present study, the more selective reduction of Trkb in immature DGCs also causes a significant increase in the number of symmetrical (GABAergic) synapses, an effect associated with an enhanced frequency of spontaneous and miniature GABAergic events in the CA3 principal cells. The increased number of GABAergic synapses in our model, Trkb Gad1-KO mice, may also be related to 
the selective reduction of the cation-chloride co-transporter NKCC1. In line with our results, previous studies using rat hippocampal cultures (Chudotvorova et al., 2005) and retinotectal neurons of the Xenopus visual system (Akerman and Cline, 2006) have demonstrated that a premature reduction of $\left[\mathrm{Cl}^{-}\right]_{i}$ by the early expression of the chloride exporter KCC2 enhances GABAergic innervation.

However, in agreement with the fact that GABAergic input remains shunting onto CA3 hippocampal interneurons throughout development (Banke and McBain, 2006), the increased release of GABA with an equilibrium potential close to the RMP observed in our model would exert a shunting inhibition at the network level with consequent disruption of GDPs leading to severe alterations in the CA3 pyramidal cell dendrites and impairment of synaptic plasticity processes and high cognitive functions. For shunting inhibition, the opening of synaptic and extrasynaptic $\mathrm{GABA}_{A}$ receptors by the phasic and tonic release of GABA, respectively, leads to a decrease in membrane resistance with a consequent reduction in the efficacy of excitatory signals that will be unable to reach the threshold for action potential generation (Loscher et al., 2013). Altogether, these data suggest a critical role for TrkB signaling regulating early spontaneous activity, fundamental for the establishment of functional neuronal circuits in vivo and animal behavior in adulthood. Moreover, at immature Schaffer collateral-CA1 synapses, GDP-induced LTP strictly depends on BDNF. This acts on presynaptic and postsynaptic TrkB receptors to enhance the probability of glutamate release and to activate the MAPK/ERK signaling pathway, respectively, leading to transcriptional regulation and new protein synthesis in postsynaptic neurons (Mohajerani et al., 2007).

It is renowned that TrkB and its ligand, BDNF, are critical regulators of adult hippocampal synaptic plasticity and learning (Cohen-Cory et al., 2010; Lu et al., 2005; Minichiello, 2009; Musumeci et al., 2009). In particular, in a previous study, deletion of Trkb from forebrain principal cells that include the DGCs, starting at around P20 (Trkb-CaMKII-CRE) after the majority of granule cells have reached and integrated into the $\mathrm{GCL}$, did not induce morphological and structural defects (Minichiello et al., 1999). Homozygous mutants showed impaired hippocampal LTP and increasingly impaired learning primarily when facing complex or stressful learning paradigms. For example, in contextual FC, Trkb-CaMKII-CRE mice showed retarded acquisition of the freezing response by impaired contextual response immediately after the acquisition phase, but proper freezing after $24 \mathrm{~h}$, indicating a short-term synaptic plasticity deficit involving both the hippocampus and proximally connected forebrain structures. In the present study, depletion of TrkB signaling from immature DGCs early in postnatal development impaired morphological maturation of neurons measured by dendritic branching and spine formation with consequent alteration of adult hippocampal synaptic plasticity and learning. In the FC paradigm mutants showed decreased acquisition of the freezing response during the training phase and impaired recognition of the neutral context after $24 \mathrm{~h}$, but a normal response to a neutral stimulus (tone), suggesting hippocampal-dependent long-term memory deficit. Thus, in contrast to adult Trkb deletion, removing TrkB signaling in immature DGCs early in postnatal development affects neurodevelopmental programs leading to adult neural dysfunction. Although the Cre-mediated deletion of Trkb in the present model is selective within the hippocampus, it does occur in some GABAergic neurons of other brain areas (Ohtsuka et al., 2013). Therefore, we cannot exclude the possibility that alterations in synaptic strength can involve other brain areas connected to the hippocampus proper such as the EC and the amygdala. However, the requirement for TrkB signaling in immature DGCs is cell autonomous as indicated by genetically based experiments wherein postnatal deletion of Trkb from forebrain principal neurons including differentiated DGCs showed impaired spatial learning and synaptic plasticity, but normal brain morphology (Minichiello et al., 1999). Also, specific ablation of TrkB signaling from adult dentate neural progenitor cells (NPCs) using a Cre line enabling deletion in radial glia progenitor cells affects survival and maturation of NPCs and results in impaired response to antidepressant treatment. The latter does not occur if Trkb is deleted from the differentiated DGCs (Li et al., 2008; Minichiello et al., 1999).

Overall, this study suggests that early in postnatal development BDNF-TrkB activation in immature DGCs provides an instructive signal that drives the sequential maturation of intrinsic hippocampal circuits via the depolarizing action of GABA. Disrupting this signal at a critical period leads to neurodevelopmental defects, followed by synaptic plasticity and cognitive impairments in adulthood. The changes we have observed in Trkb Gad1-KO mouse model are recurrent pathological features shared in part by many neurodevelopmental disorders (Griguoli and Cherubini, 2017) (Deidda et al., 2014). Moreover, the present data establish the genetic importance of TrkB signaling at a critical period, shaping hippocampal neuronal circuitry by controlling the GABAergic system development. Therefore, these findings have clinical 
implication by revealing the existence of sensitive time window in which a developing brain might be more amenable to treatment and may stimulate further research into mechanisms underlying this type of disorders.

\section{Limitations of the Study}

Our data establish the genetic importance of TrkB signaling in immature DGCs driving the sequential development of intrinsic hippocampal circuits by modulating early GABA signaling through the expression of $N k c c 1$. It would be interesting to determine if by rescuing $N k c c 1$ expression in the postsynaptic target of DGCs, the CA3 principal cells, would be sufficient to revert or prevent the phenotype. Moreover, it would also be instrumental to determine the exact mechanism whereby presynaptic TrkB signaling (DGCs) affects postsynaptic Nkcc1 expression levels (CA3 principal cells).

\section{METHODS}

All methods can be found in the accompanying Transparent Methods supplemental file.

\section{DATA AND CODE AVAILABILITY}

This study did not generate datasets or code.

\section{SUPPLEMENTAL INFORMATION}

Supplemental Information can be found online at https://doi.org/10.1016/j.isci.2020.101078.

\section{ACKNOWLEDGMENTS}

This work was supported in part by grants from the European Union (EU FP6 MEMORIES, 037831; EU FP6 StemStroke, 037526) and the BBSRC (BB/L021382/1) to L.M.; from the European Union's Horizon 2020 Framework Program for Research and Innovation under the Specific Grant Agreement No. 785907 (Human Brain Project SGA2) and from Telethon, Italy (GGP 16083) to E.C.; from the MECC-BFU2014-56692-R to A.G. and J.M.D.-G. S.B. was supported by an ESTAR fellowship funded by the EC's FP6 Marie Curie host fellowship for Early Stage Research Training (MEST-CT-2004-504640). "The funders had no role in study design, data collection and analysis, decision to publish, or preparation of the manuscript."

\section{AUTHOR CONTRIBUTIONS}

S.B. and B.Z. performed behavioral and histological analysis. A.A.-M. performed histological analysis. M.G. and L.L. carried out in vitro electrophysiology analysis, E.C. provided supervision, theoretical input, and results interpretation. S.B., M.T.J.-P., A.G., and J.M.D.-G. performed in vivo electrophysiology analysis and results interpretation; T.H.G. performed the EM analysis. S.B., G.F., and B.Z. performed biochemical assays. S.M. carried out morphological analysis. L.M. contributed to the overall experimental plans, supervised the project, provided theoretical input and wrote the manuscript with comments and contributions from all authors.

\section{DECLARATION OF INTERESTS}

The authors declare no competing interests.

Received: February 14, 2020

Revised: March 23, 2020

Accepted: April 14, 2020

Published: May 22, 2020

\section{REFERENCES}

Acsady, L., Kamondi, A., Sik, A., Freund, T., and Buzsaki, G. (1998). GABAergic cells are the major postsynaptic targets of mossy fibers in the rat hippocampus. J. Neurosci. 18, 3386-3403.

Aquado, F., Carmona, M.A., Pozas, E., Aguilo, A., Martinez-Guijarro, F.J., Alcantara, S., Borrell, V.

Yuste, R., Ibanez, C.F., and Soriano, E. (2003).
BDNF regulates spontaneous correlated activity at early developmental stages by increasing synaptogenesis and expression of the $\mathrm{K}+/ \mathrm{Cl}-\mathrm{co}$ transporter KCC2. Development 130, 1267-1280.

Akerman, C.J., and Cline, H.T. (2006).

Depolarizing GABAergic conductances regulate the balance of excitation to inhibition in the developing retinotectal circuit in vivo. J. Neurosci. 26, 5117-5130.

Banke, T.G., and McBain, C.J. (2006). GABAergic input onto CA3 hippocampal interneurons remains shunting throughout development. J. Neurosci. 26, 11720-11725. 
Ben-Ari, Y. (2002). Excitatory actions of gaba during development: the nature of the nurture. Nat. Rev. Neurosci. 3, 728-739.

Ben-Ari, Y Khalilov, I., Kahle, K.T., and Cherubini, E. (2012). The GABA excitatory/inhibitory shift in brain maturation and neurological disorders. Neuroscientist 18, 467-486.

Berg, D.A., Su, Y., Jimenez-Cyrus, D., Patel, A., Huang, N., Morizet, D., Lee, S., Shah, R., Ringeling, F.R., Jain, R., et al. (2019). A common embryonic origin of stem cells drives developmental and adult neurogenesis. Cell 177 654-668 e615.

Blaesse, P., Airaksinen, M., Rivera, C., and Kaila, K. (2009). Cation-chloride cotransporters and neuronal function. Neuron 61, 820-838.

Brickley, S.G., and Mody, I. (2012). Extrasynaptic GABA(A) receptors: their function in the CNS and implications for disease. Neuron 73, 23-34.

Brown, J.P. Couillard-Despres, S., Cooper-Kuhn C.M., Winkler, J., Aigner, L., and Kuhn, H.G. (2003). Transient expression of doublecortin during adult neurogenesis. J. Comp. Neurol. 467, $1-10$.

Cabezas, C., Irinopoulou, T., Cauli, B., and Poncer, J. (2013). Molecular and functional characterization of GAD67-expressing, newborn granule cells in mouse dentate gyrus. Front. Neural Circuits 7, 60.

Cancedda, L., Fiumelli, H., Chen, K., and Poo, M.M. (2007). Excitatory GABA action is essential for morphological maturation of cortical neurons in vivo. J. Neurosci. 27, 5224-5235.

Cellot, G., Maggi, L., Di Castro, M.A., Catalano, M., Migliore, R., Migliore, M., Scattoni, M.L., Calamandrei, G., and Cherubini, E. (2016). Premature changes in neuronal excitability account for hippocampal network impairment and autistic-like behavior in neonatal BTBR T+tf/J mice. Sci. Rep. 6, 31696.

Chudotvorova, I., Ivanov, A., Rama, S., Hubner, C.A., Pellegrino, C., Ben-Ari, Y., and Medina, I. (2005). Early expression of KCC2 in rat hippocampal cultures augments expression of functional GABA synapses. J. Physiol. 566, 671-679.

Cohen-Cory, S., Kidane, A.H., Shirkey, N.J., and Marshak, S. (2010). Brain-derived neurotrophic factor and the development of structural neuronal connectivity. Dev. Neurobiol. 70, 271-288.

Danzer, S.C., Kotloski, R.J., Walter, C., Hughes, M., and McNamara, J.O. (2008). Altered morphology of hippocampal dentate granule cell presynaptic and postsynaptic terminals following conditional deletion of TrkB. Hippocampus 18, 668-678.

Deidda, G., Bozarth, I.F., and Cancedda, L. (2014). Modulation of GABAergic transmission in development and neurodevelopmenta disorders: investigating physiology and pathology to gain therapeutic perspectives. Front. Cell Neurosci. 8, 119

Drake, C.T., Milner, T.A., and Patterson, S.L. (1999). Ultrastructural localization of full-length trkB immunoreactivity in rat hippocampus suggests multiple roles in modulating activitydependent synaptic plasticity. J. Neurosci. 19, 8009-8026.

Durand, G.M., Kovalchuk, Y, and Konnerth, A (1996). Long-term potentiation and functional synapse induction in developing hippocampus. Nature 381, 71-75.

Fiumelli, H., and Woodin, M.A. (2007). Role of activity-dependent regulation of neuronal chloride homeostasis in development. Curr. Opin. Neurobiol. 17, 81-86.

Ge, S., Goh, E.L., Sailor, K.A., Kitabatake, Y., Ming, G.L., and Song, H. (2006). GABA regulates synaptic integration of newly generated neurons in the adult brain. Nature 439, 589-593.

Ge, S., Yang, C.H., Hsu, K.S., Ming, G.L., and Song, H. (2007). A critical period for enhanced synaptic plasticity in newly generated neurons of the adult brain. Neuron 54, 559-566.

Gottmann, K., Mittmann, T, and Lessmann, V. (2009). BDNF signaling in the formation, maturation and plasticity of glutamatergic and GABAergic synapses. Exp. Brain Res. 199, 203-234.

Griguoli, M., and Cherubini, E. (2017). Early correlated network activity in the Hippocampus: its putative role in shaping neuronal circuits. Front. Cell Neurosci 11,255.

Hochgerner, H., Zeisel, A., Lonnerberg, P., and Linnarsson, S. (2018). Conserved properties of dentate gyrus neurogenesis across postnatal development revealed by single-cell RNA sequencing. Nat. Neurosci. 21, 290-299.

Hong, E.J., McCord, A.E., and Greenberg, M.E. (2008). A biological function for the neuronal activity-dependent component of Bdnf transcription in the development of cortical inhibition. Neuron 60, 610-624.

Huang, Z.J., Kirkwood, A., Pizzorusso, T. Porciatti, V. Morales, B., Bear, M.F., Maffei, L. and Tonegawa, S. (1999). BDNF regulates the maturation of inhibition and the critical period of plasticity in mouse visual cortex. Cell 98, 739-755.

Jagasia, R., Steib, K., Englberger, E., Herold, S., Faus-Kessler, T., Saxe, M., Gage, F.H., Song, H. and Lie, D.C. (2009). GABA-cAMP response element-binding protein signaling regulates maturation and survival of newly generated neurons in the adult hippocampus. J. Neurosci. $29,7966-7977$

Khazipov, R., Khalilov, I., Tyzio, R., Morozova, E. Ben-Ari, Y., and Holmes, G.L. (2004) Developmental changes in GABAergic actions and seizure susceptibility in the rat hippocampus. Eur. J. Neurosci. 19, 590-600.

Lee, I., and Kesner, R.P. (2004). Differential contributions of dorsal hippocampal subregions to memory acquisition and retrieval in contextual fear-conditioning. Hippocampus 14, 301-310.

Li, Y., Luikart, B.W., Birnbaum, S., Chen, J., Kwon, C.H. Kernie, S.G. Bassel-Duby, R., and Parada, L.F. (2008). TrkB regulates hippocampal neurogenesis and governs sensitivity to antidepressive treatment. Neuron 59, 399-412.
Loscher, W., Puskarjov, M., and Kaila, K. (2013). Cation-chloride cotransporters NKCC1 and KCC2 as potential targets for novel antiepileptic and antiepileptogenic treatments.

Neuropharmacology 69, 62-74.

Lu, B., Pang, P.T., and Woo, N.H. (2005). The yin and yang of neurotrophin action. Nat. Rev. Neurosci. 6, 603-614.

Madisen, L., Zwingman, T.A., Sunkin, S.M., Oh, S.W., Zariwala, H.A., Gu, H., Ng, L.L., Palmiter, R.D., Hawrylycz, M.J., Jones, A.R., et al. (2010). A robust and high-throughput $\mathrm{Cre}$ reporting and characterization system for the whole mouse brain. Nat. Neurosci. 13, 133-140.

Meredith, R.M., Dawitz, J., and Kramvis, I. (2012) Sensitive time-windows for susceptibility in neurodevelopmental disorders. Trends Neurosci. 35, 335-344.

Minichiello, L. (2009). TrkB signalling pathways in LTP and learning. Nat. Rev. Neurosci. 10, 850-860.

Minichiello, L., Korte, M., Wolfer, D., Kühn, R., Unsicker, K., Cestari, V., Rossi-Arnaud, C., Lipp, H.P. Bonhoeffer, T., and Klein, R. (1999). Essential role for TrkB receptors in hippocampus-mediated learning. Neuron 24, 401-414.

Mohajerani, M., Sivakumaran, S., Zacchi, P., Aguilera, P., and Cherubini, E. (2007). Correlated network activity enhances synaptic efficacy via BDNF and the ERK pathway at immature CA3 CA1 connections in the hippocampus. Proc. Natl. Acad. Sci. U S A 104, 13176-13181.

Musumeci, G., Sciarretta, C., Rodriguez-Moreno, A., Al Banchaabouchi, M., Negrete-Diaz, V., Costanzi, M., Berno, V., Egorov, A.V., von Bohlen Und Halbach, O., Cestari, V., et al. (2009). TrkB modulates fear learning and amygdalar synaptic plasticity by specific docking sites. J. Neurosci. 29, 10131-10143.

Nicola, Z., Fabel, K., and Kempermann, G. (2015). Development of the adult neurogenic niche in the hippocampus of mice. Front. Neuroanat. 9, 53.

Nicoll, R.A., and Schmitz, D. (2005). Synaptic plasticity at hippocampal mossy fibre synapses. Nat. Rev. Neurosci. 6, 863-876.

Novak, A., Guo, C., Yang, W., Nagy, A., and Lobe, C.G. (2000). Z/EG, a double reporter mouse line that expresses enhanced green fluorescent protein upon Cre-mediated excision. Genesis 28 147-155.

Ohtsuka, N., Badurek, S., Busslinger, M., Benes, F., Minichiello, L., and Rudolph, U. (2013). GABAergic neurons regulate lateral ventricular development via transcription factor Pax5 Genesis 51, 234-245.

Owens, D.F., and Kriegstein, A.R. (2002). Is there more to GABA than synaptic inhibition? Nat. Rev. Neurosci. 3, 715-727.

Pfeffer, C.K., Stein, V., Keating, D.J., Maier, H. Rinke, I., Rudhard, Y , Hentschke, M. Rune, G.M. Jentsch, T.J., and Hubner, C.A. (2009). NKCC1dependent GABAergic excitation drives synaptic network maturation during early hippocampal development. J. Neurosci. 29, 3419-3430. 
Porter, A.G., and Janicke, R.U. (1999). Emerging roles of caspase-3 in apoptosis. Cell Death Differ. 6, 99-104.

Rivera, C., Voipio, J., Payne, J.A., Ruusuvuori, E., Lahtinen, H., Lamsa, K., Pirvola, U., Saarma, M. and Kaila, K. (1999). The K+/Cl- co-transporter KCC2 renders GABA hyperpolarizing during neuronal maturation. Nature 397, 251-255.

Safiulina, V., Fattorini, G., Conti, F., and Cherubini, E. (2006). GABAergic signaling at mossy fiber synapses in neonatal rat hippocampus. J. Neurosci. 26, 597-608.

Safiulina, V.F., Zacchi, P., Taglialatela, M., Yaari, Y., and Cherubini, E. (2008). Low expression of $\mathrm{Kv} 7 / \mathrm{M}$ channels facilitates intrinsic and network bursting in the developing rat hippocampus.

J. Physiol. 586, 5437-5453.
Scholzen, T., and Gerdes, J. (2000). The Ki-67 protein: from the known and the unknown. J. Cell Physiol. 182, 311-322.

Sipila, S.T., Schuchmann, S., Voipio, J., Yamada, J., and Kaila, K. (2006). The cation-chloride cotransporter NKCC1 promotes sharp waves in the neonatal rat hippocampus. J. Physiol. 573, 765-773.

Sivakumaran, S., Mohajerani, M.H., and Cherubini, E. (2009). At immature mossy-fiberCA3 synapses, correlated presynaptic and postsynaptic activity persistently enhances GABA release and network excitability via BDNF and cAMP-dependent PKA. J. Neurosci. 29, 26372647.

Tozuka, Y., Fukuda, S., Namba, T., Seki, T., and Hisatsune, T. (2005). GABAergic excitation promotes neuronal differentiation in adult hippocampal progenitor cells. Neuron 47, 803-815.

von Bohlen Und Halbach, O. (2007).

Immunohistological markers for staging neurogenesis in adult hippocampus. Cell Tissue

Res. 329, 409-420.

Wilson, D.I., Langston, R.F., Schlesiger, M.I., Wagner, M., Watanabe, S., and Ainge, J.A. (2013) Lateral entorhinal cortex is critical for novel object-context recognition. Hippocampus 23, 352-366.

Yamada, M.K., Nakanishi, K., Ohba, S., Nakamura, T., Ikegaya, Y., Nishiyama, N., and Matsuki, N. (2002). Brain-derived neurotrophic factor promotes the maturation of GABAergic mechanisms in cultured hippocampal neurons. J. Neurosci. 22, 7580-7585. 
iScience, Volume 23

Supplemental Information

Immature Dentate Granule Cells

Require Ntrk2/Trkb for the Formation

of Functional Hippocampal Circuitry

Sylvia Badurek, Marilena Griguoli, Aman Asif-Malik, Barbara Zonta, Fei Guo, Silvia Middei, Laura Lagostena, Maria Teresa Jurado-Parras, Thomas H. Gillingwater, Agnès Gruart, José María Delgado-García, Enrico Cherubini, and Liliana Minichiello 


\section{Supplemental Figures}
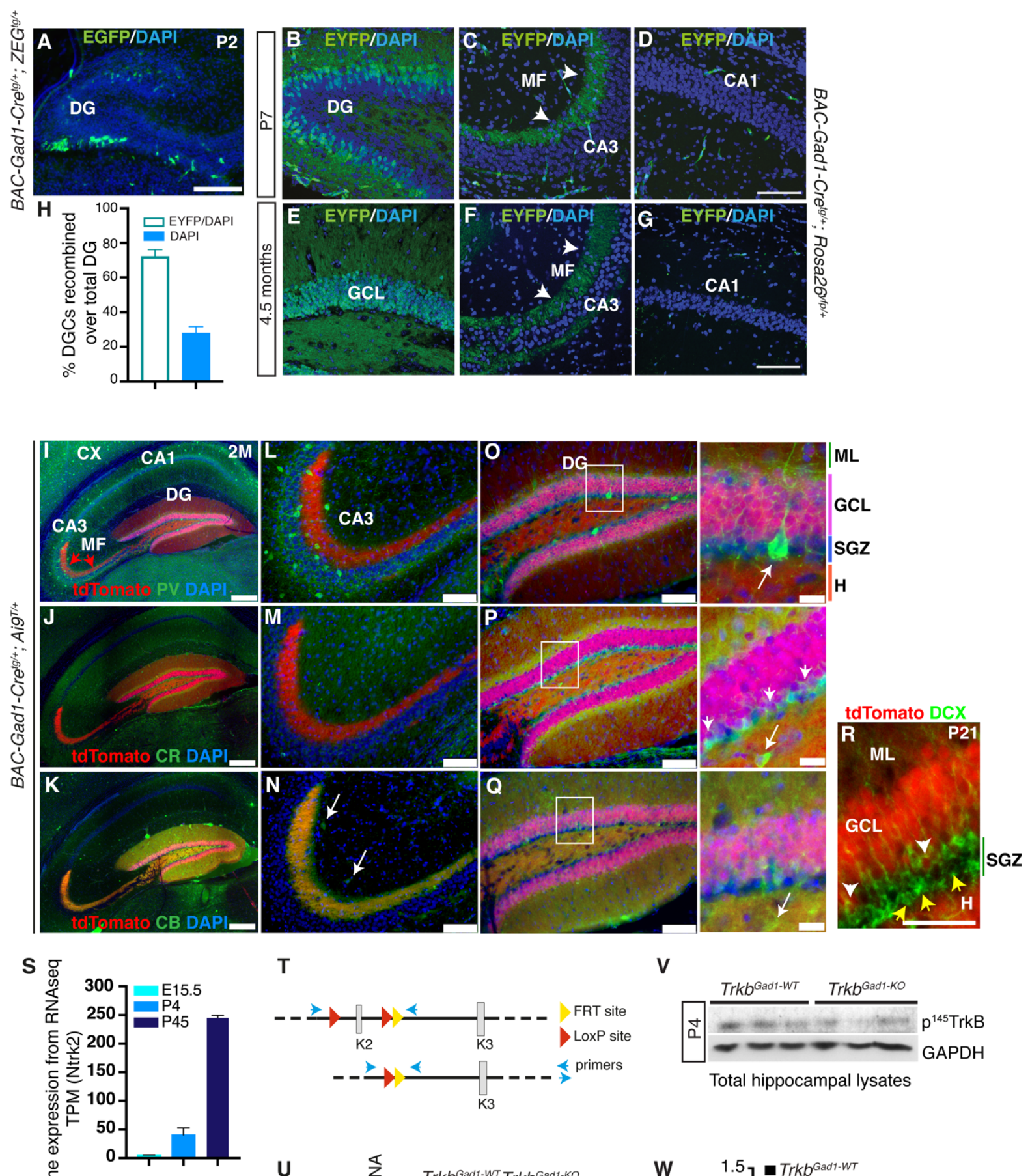

$\mathbf{T}$

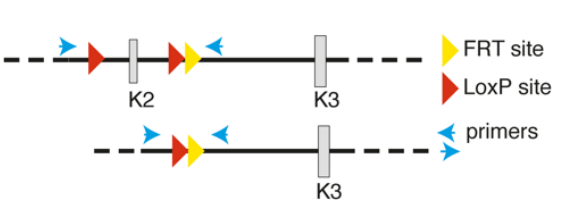

V
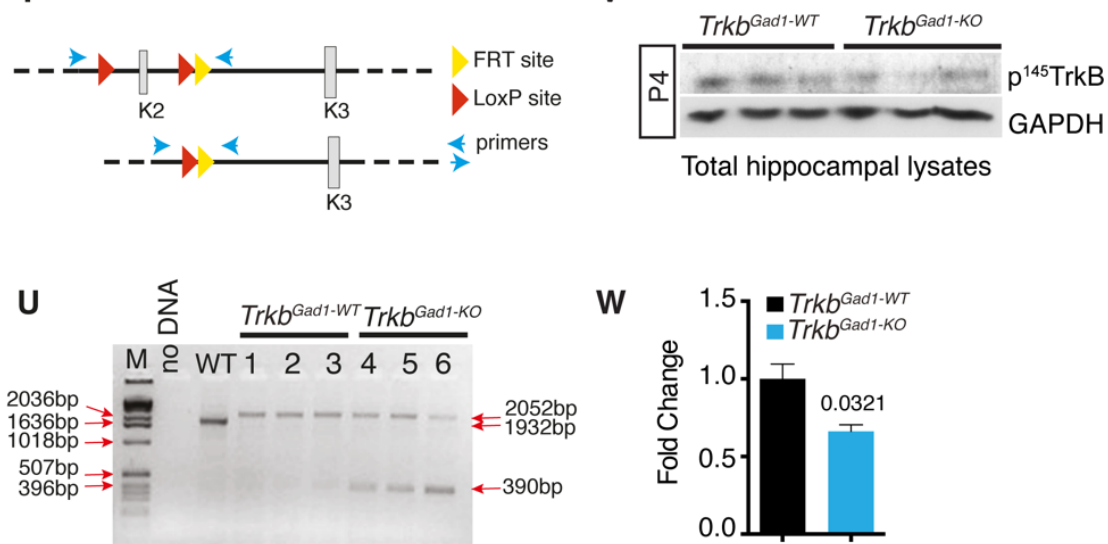

Figure S1. Gad1-Cre recombination is specific to immature dentate granule cells within the hippocampus. Related to Figure 1.

(A-R) BAC-Gad1-Cre line expression pattern within the hippocampal formation revealed by crossing the Cre line to various reporter strains. (A) Representative sagittal hippocampal 
sections from a P2 BAC-Gad1-Cre ${ }^{t g /+} ; Z / E G^{t g /+}$ mouse. The reporter signal, EGFP (green), and DAPI-stained nuclei (blue) were superimposed. (B-H) Representative sagittal hippocampal sections from P7 (B-D) and 4.5M (E-G) BAC-Gad1-Cre $e^{t g+/}$; $R 26 R^{y f p /+}$ mice. The reporter signal, EYFP (green), and DAPI-stained nuclei (blue) were superimposed. In the CA3 region, mossy fibres (arrowheads) show the EYFP signal as a result of the recombination in the dentate gyrus granule cells.

(H) Quantification of EYFP-expressing cells versus total DG cells in adulthood (4.5M). Total DG cells were identified by DAPI staining. The percent of EYFP positive DGCs over the total number of DAPI-stained DG nuclei: $72 \pm 4 \%$ SEM. N=3 mice (on average 2 sections per mouse were used).

(I-K) Representative coronal sections from 2M old BAC-Gad1-Cre ${ }^{\operatorname{tg} /+} ; A i 9 R /+$ mouse brain $(\mathrm{n}=2)$, highlighting prominent tdTomato fluorescent signal (red) in dentate granule neurons and the MF terminals (arrowheads), were immunostained with primary antibodies against Parvalbumin (PV), Calretinin (CR), and Calbindin (CB), (green) respectively. The green signal was superimposed to tdTomato and DAPI signals. No colocalization was observed between each primary antibody (green) and tdTomato signal in interneurons (arrows) both in the CA3 region and the DG (L-Q and respective insets); however, a colocalization was observed between tdTomato signal and Calretinin (green) in immature granule cells within the SGZ (P and inset, arrowheads), and as expected, between tdTomato and Calbindin (green) in mature granule cells in the GCL ( $\mathbf{Q}$ and inset).

(R) Representative coronal hippocampal sections from a P21 BAC-Gad1-Cre $e^{\operatorname{tg}+}$; $\mathrm{Ai9}^{T /+}$ mice. tdTomato reporter signal (red) and DCX immunostaining (green) were superimposed. White arrowheads indicate examples of colocalization between tdTomato and DCX occurring only in immature neurons (DGCs beginning to migrate and mature in the GCL) but not in neural precursors (yellow arrows).

(S) Gene expression data expressed in transcripts per million (TPM) and extracted from 'Table 2' in BERG ET AL., 2019, (Berg et al., 2019) showing developmental upregulation of Ntrk2/Trkb in dentate neural progenitors at different stages (E15.5, P4 and P45); qval 0.0456 for E15.5 vs P4; and 0.0051 for P4 vs P45.

(T-U) PCR strategy to detect Trkb recombination. (T) Schematic representation of Trkb floxed allele before and after Cre-mediated recombination. (U) Trkb recombination by PCR strategy. Genomic DNA was isolated from adult hippocampus. The strategy chosen amplifies a PCR product of 2052bp from the non-recombined Trkb floxed allele, a 1932bp from the wild-type Trkb allele, and a 390bp from the Trkb floxed allele after Cre-mediated recombination. The recombined band (390bp) was detected in every sample from Trkb ${ }^{G a d l-K O}$ mice together with a faint 2052bp band for the un-recombined allele. Control mice, which carry Trkb floxed alleles but not Gad1-Cre transgene, showed only the larger PCR products resulting from the unrecombined Trkb floxed allele. The WT sample showed only a wild-type Trkb allele size band (1932bp).

(V-W) Western blot analysis (V) and quantification of fold changes (W) from P4 total hippocampal lysates shows significantly reduced TrkB full length in Trkb Gadl-KO mice compared to $\operatorname{Trkb} b^{\text {Gadl-WT }}\left(\operatorname{Trkb} b^{\text {Gadl-WT }}, \mathrm{n}=3: 1.000 \pm 0.09576, \operatorname{Trkb} b^{\text {Gadl-KO }}, \mathrm{n}=3: 0.6615 \pm\right.$ $0.04303, P=0.0321$ ). 
DG, dentate gyrus; MF, mossy fibres, GCL, granule cell layer; CX, cortex; DG, dentate gyrus, MF, mossy fibers. ML, molecular layer; GCL, granular cell layer; SGZ, subgranular zone; H, hilus. DCX, Doublecortin. Scale bars A-G $(100 \mu \mathrm{m})$; I-K $(250 \mu \mathrm{m})$; L-Q $(100 \mu \mathrm{m})$; insets $(10 \mu \mathrm{m}) ; \mathrm{R}(25 \mu \mathrm{m})$. 

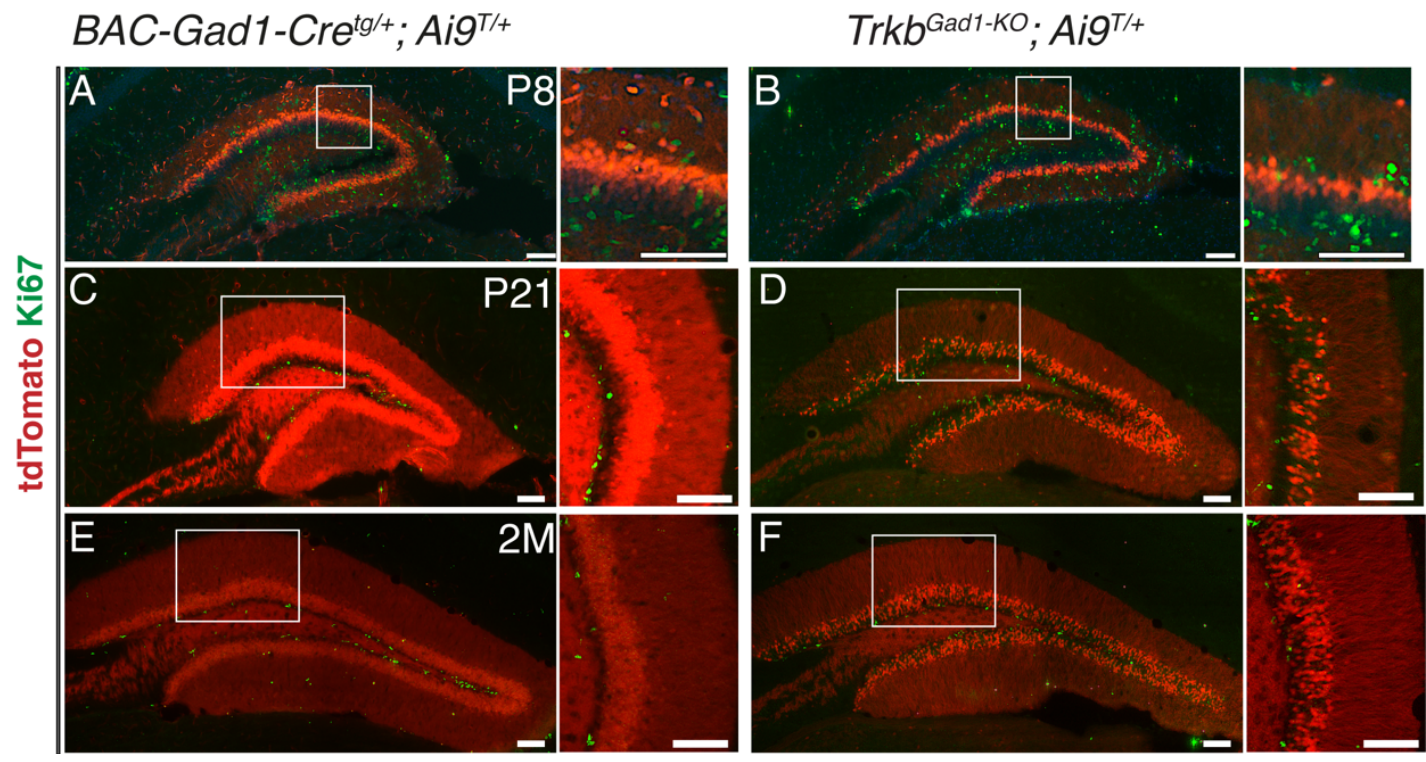

Figure S2. Similar proliferation of dentate progenitors between mutants and controls. Related to Figure 2.

(A-F) Immunostaining for Ki67 (green) shows similar pattern of dentate progenitor proliferation between controls ( $\left.\mathrm{BAC}-\mathrm{Gadl}-\mathrm{Cr}^{\mathrm{tg} /+} ; \mathrm{Ai} \mathrm{9}^{\mathrm{T/+}}\right)$ and mutants $\left(\operatorname{Trkb}^{\mathrm{Gadl}-\mathrm{KO}} ; \mathrm{Ai}^{\mathrm{T/+}}\right)$ at all ages analysed, P8 (A-B, and respective insets), P21 (C-D, and respective insets) and 2M (E-F, and respective insets). Scale bars, A-F, and respective insets $(100 \mu \mathrm{m})$. 
A

Trkb $\quad T r k b^{G a d 1-W T}$
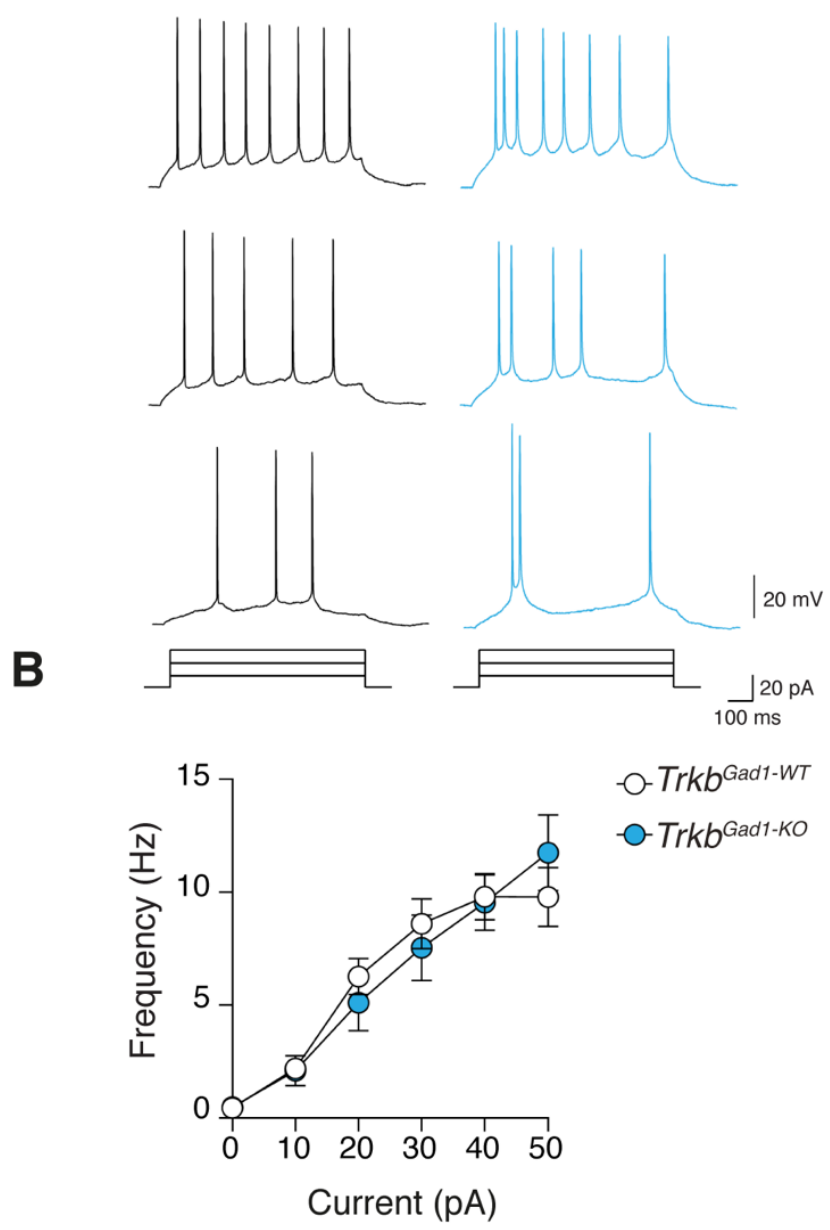

Figure S3. Neuronal excitability is unchanged in $\operatorname{Trkb}^{\text {Gad1-KO }}$ mice. Related to Figure 3.

(A) Sample traces showing the firing activity induced in CA3 principal cells by depolarizing current pulses of increasing amplitude (delivered in the presence of AMPA, NMDA and $\mathrm{GABA}_{\mathrm{A}}$ receptor antagonists) from control littermate and $\operatorname{Trkb} b^{\text {Gadl-KO }}$ mice.

(B) Each point represents the mean spike frequency $(\mathrm{Hz})$ versus the amount of injected current (pA) in control and in Trkb $b^{G a d l-K O}$ mice. No significant differences were observed between the two genotypes. 

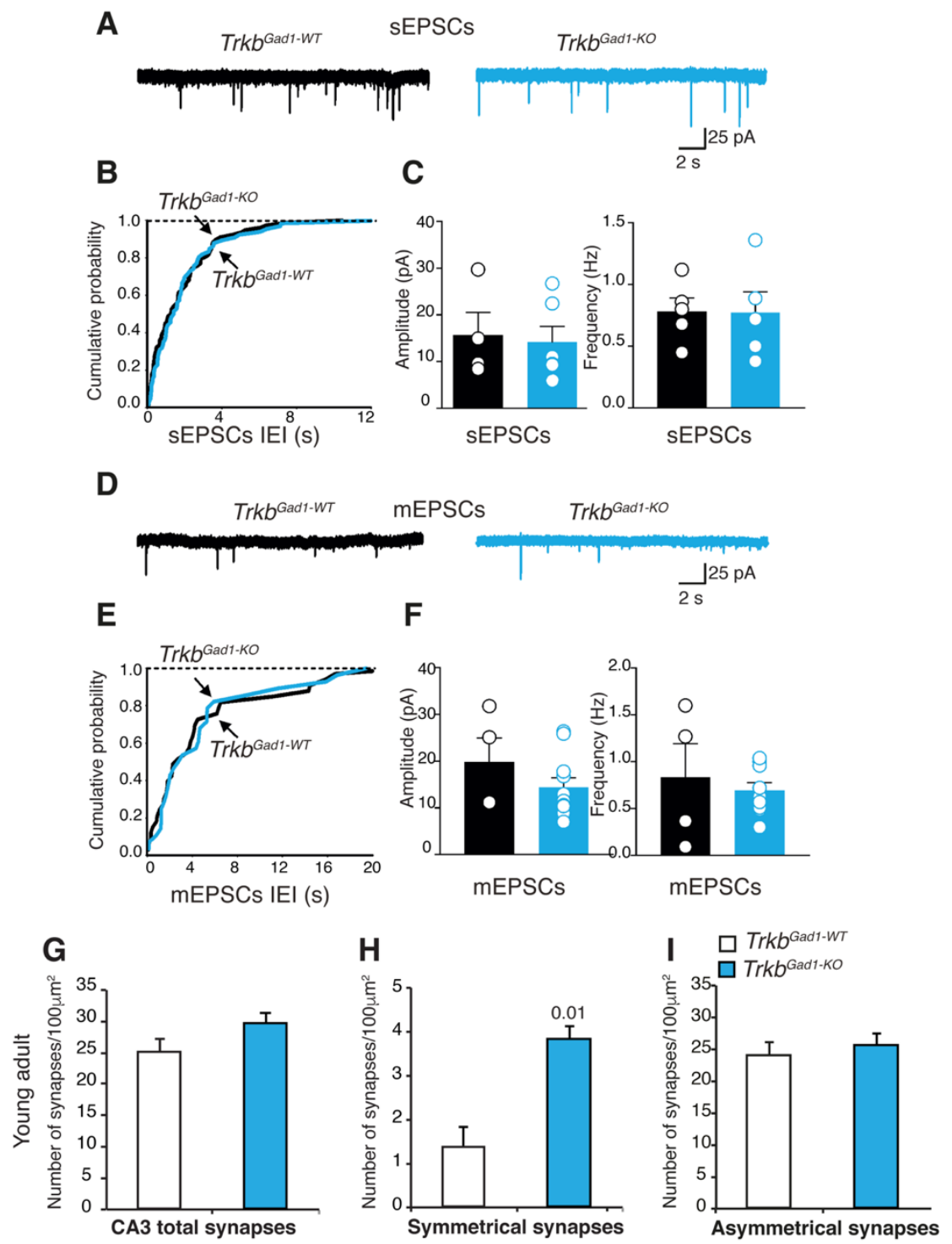

Figure S4. Spontaneous and miniature AMPA-mediated postsynaptic currents (sEPSCs and mEPSCs) are not affected in Trkb ${ }^{\text {Gad1-KO}}$ mice. Related to Figure 3.

(A) SEPSCs recorded in isolation (in the presence of bicuculline, DL-APV to block GABA and NMDA receptors, respectively) from CA3 principal cells in hippocampal slices from P5P9 in control (Trkb $b^{\text {Gadl-WT}}$, black) and in Trkb $b^{\text {Gadl-KO }}$ mice (cyan).

(B). Cumulative distribution of inter event interval (IEI) for cells shown in (A).

(C). Summary plots of mean amplitude and frequency of sEPSCs shown in (B); sEPSCs [amplitude, Trkb $b^{\text {Gadl-WT }}, 17 \pm 4 \mathrm{pA}$ ( $\mathrm{n}=5$ from 2 pups) vs. Trkb ${ }^{\text {Gadl-KO }}, 14 \pm 3 \mathrm{pA}$ (n=7 from

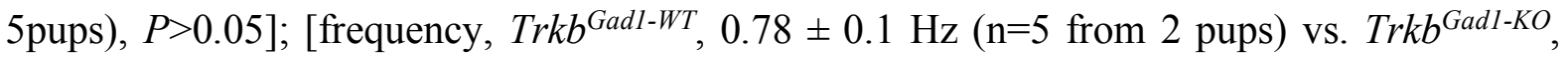
$0.77 \pm 0.17$ ( $\mathrm{n}=7$ from 5 pups), $P>0.05]$.

(D-F) as in (A-B) but for mEPSCs [amplitude, Trkb $b^{\text {Gadl-WT }}, 20 \pm 8$ pA (n=4 from 2 pups) vs. $\operatorname{Trkb} b^{\text {Gadl-KO }}, 14 \pm 3 \mathrm{pA}$ (n=11 from 5 pups), $P>0.05$ ]; [frequency, Trkb $b^{\text {Gadl-WT } 0.83 \pm 0.35 \mathrm{~Hz}}$ ( $\mathrm{n}=4$ from 2 pups) vs. Trkb ${ }^{\text {Gadl-KO }} 0.69 \pm 0.25 \mathrm{~Hz}$ ( $\mathrm{n}=11$ from 5 pups), $\left.P>0.05\right]$. Values are mean \pm SEM. $P$ statistic from unpaired Student's $t$-tests. 
(G-I) Symmetrical synapses remain increased in the young adult Trkb ${ }^{\text {Gadl-KO }} \mathrm{CA} 3$ hippocampal region. Number of synapses in the CA3 hippocampal region $/ 100 \mu \mathrm{m}^{2}$, two months old mice

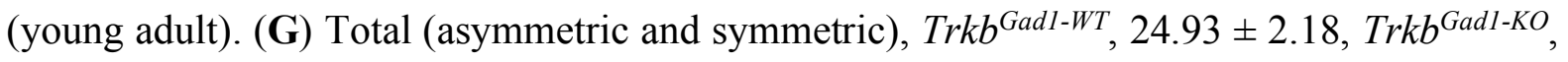
$29.56 \pm 1.88, \mathrm{P}>0.05$. (H) Symmetrical synapses, Trkb $b^{\text {Gadl-WT }}, 1.34 \pm 0.47$ vs. Trkb $b^{\text {Gadl-KO }}, 3.83$ $\pm 0.29, \mathrm{P}=0.01$. (I) Asymmetrical synapses, Trkb $b^{\text {Gadl-WT }}, 24.00 \pm 2.46 \mathrm{vs} \operatorname{Trkb}{ }^{\text {Gadl-KO}}, 25.74 \pm$ 1.95, $\mathrm{P}>0.05$. Trkb $b^{\text {Gadl-WT }}(\mathrm{n}=6), \operatorname{Trkb} b^{\text {Gadl-KO }}(\mathrm{n}=3)$. Values are mean $\pm \mathrm{SEM}, \mathrm{P}$ statistic from unpaired one-tailed Student's t-tests. 
A
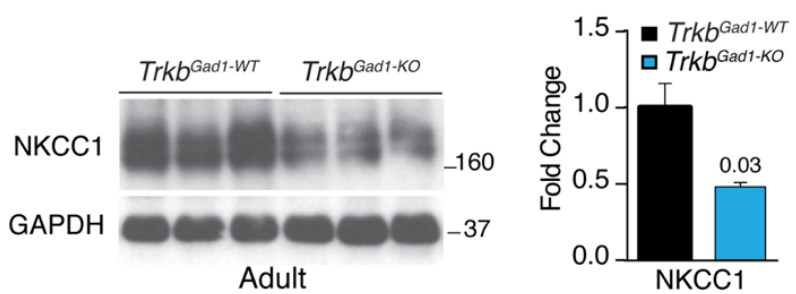

B
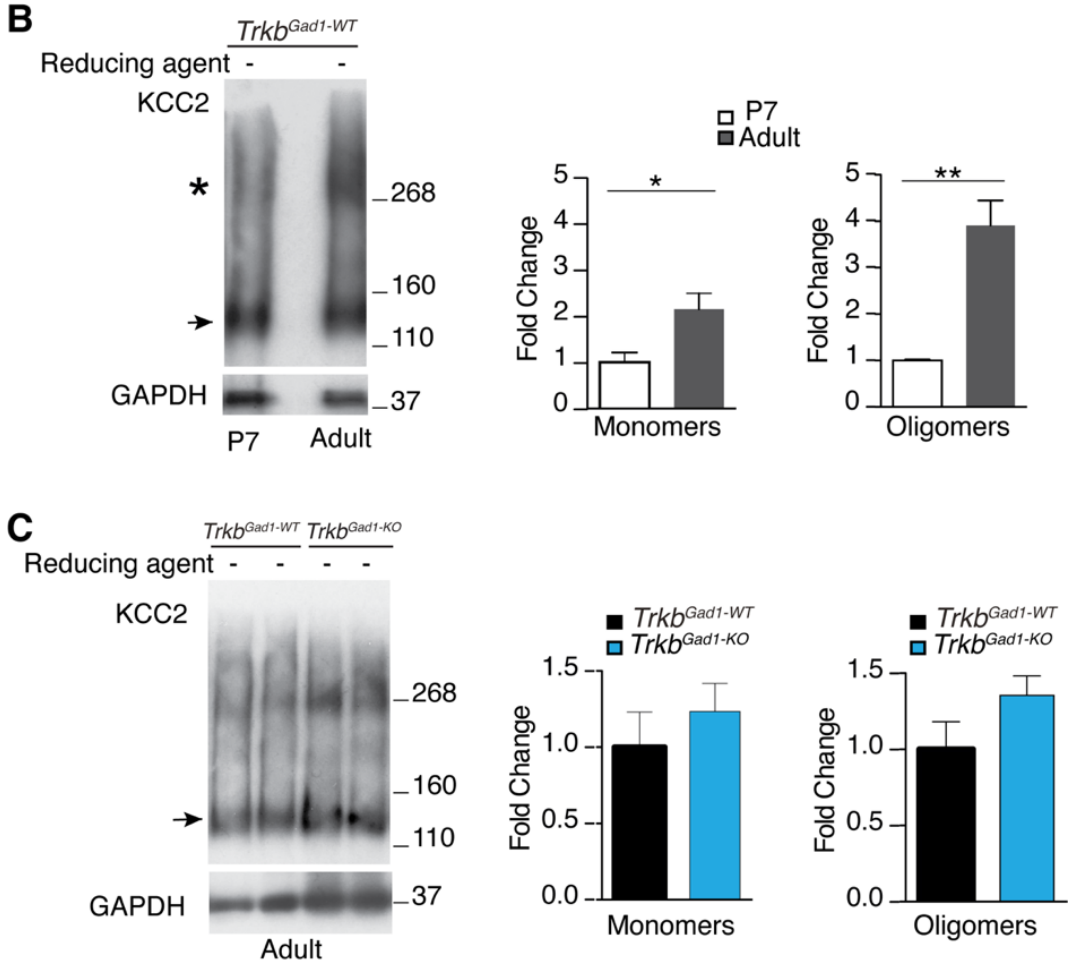

Figure S5. NKCC1 but not KCC2 expression is altered in the hippocampus of adult Trkb $\boldsymbol{B a d 1 - K O}^{\text {mice. Related to Figure } 4 .}$

(A) Representative western blots (WB) from adult (P50) hippocampal lysates and relative quantification of NKCC1 protein levels [P50 Control $\left(\right.$ Trkb $\left.{ }^{\text {Gadl-WT }}\right)(\mathrm{n}=6), 1.000 \pm 0.1557$, $\left.\operatorname{Trkb} b^{\text {Gadl-KO }}(\mathrm{n}=6): 0.4815 \pm 0.02869, P=0.03\right]$.

(B-C) Representative WB from P7 and P50 hippocampal protein lysates and relative quantification of KCC2 monomers and oligomers. (B) Protein lysates were not treated with a reducing agent and were separated by a linear 3-8\% Tris-acetate NuPAGE gel to detect KCC2 oligomers. Distinct KCC2 immunoreactive bands around 130 and $270 \mathrm{kDa}$ were detected mainly at P50. Arrow denotes the monomeric $\mathrm{KCC} 2$, and the asterisk points to $\mathrm{KCC} 2$ oligomers. Quantification reveals significant developmental increase between P7 and P50 of both monomers (Trkb Gad1-WT $, \mathrm{P} 7(\mathrm{n}=3), 1.000 \pm 0.2142 ; \operatorname{Trkb}^{\text {Gadl-WT }}, \mathrm{P} 50(\mathrm{n}=3), 2.157 \pm 0.3415$, $P=0.045)$ and oligomers (Trkb ${ }^{\text {Gadl-WT }}, \mathrm{P} 7(\mathrm{n}=3), 1.000 \pm 0.02456 ; \operatorname{Trk}^{\text {Gadl-WT }} \mathrm{P} 50(\mathrm{n}=3), 3.909$ $\pm 0.5392, P=0.0057)$. (C) Quantification of KCC2 monomers and oligomers at P50 between mutants and controls (monomers, Trkb ${ }^{\text {Gad1-WT }}(\mathrm{n}=3), 1.000 \pm 0.2306 ; \operatorname{Trkb}^{\text {Gadl-KO}}(\mathrm{n}=3), 1.234$ $\pm 0.1837, P=0.47$; oligomers, $\operatorname{Trk} b^{\text {Gadl-WT }}(\mathrm{n}=3), 1.000 \pm 0.1810 ; \operatorname{Trk} b^{\text {Gadl-KO }}(\mathrm{n}=3), 1.355 \pm$ $0.1273, P=0.18)$. GAPDH was used as loading control. Values are mean \pm SEM. $P$ statistic from unpaired Student's $t$-test. 
A

$\Delta \operatorname{Trkb}^{\mathrm{Gad1}-W T}$
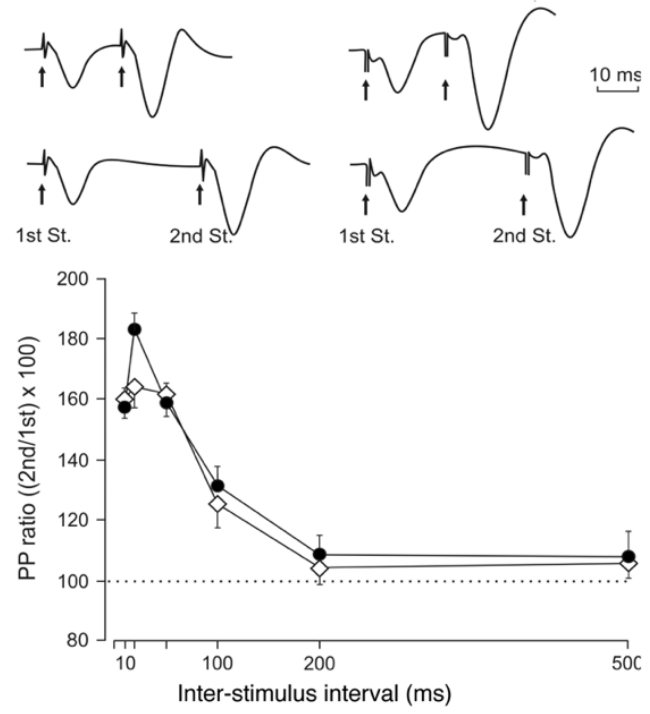

C

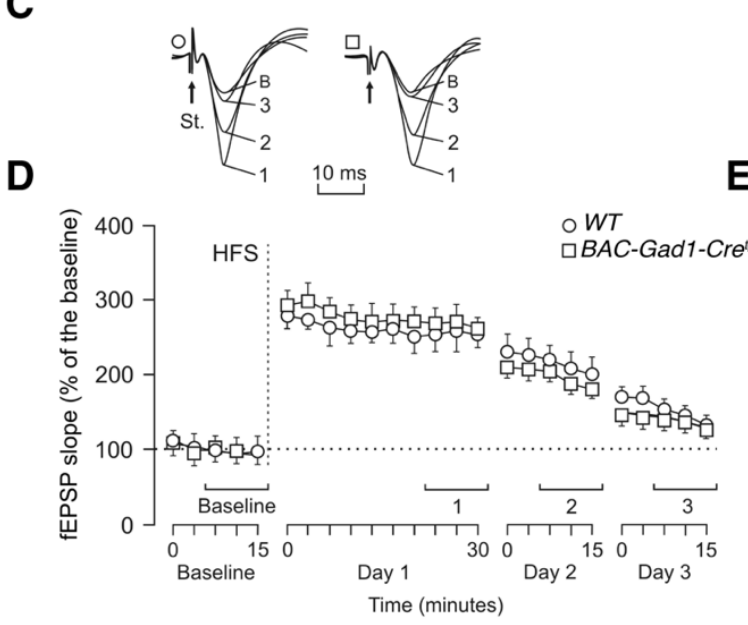

B
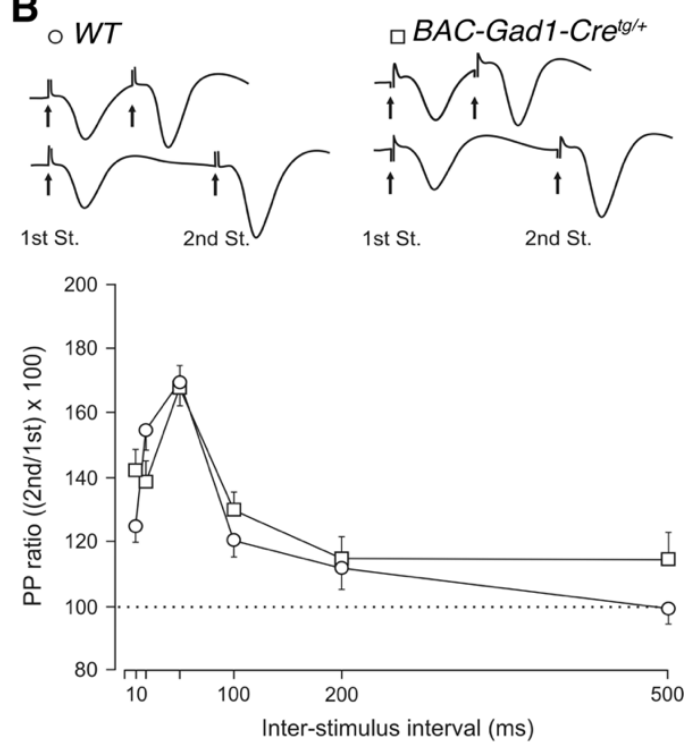

E

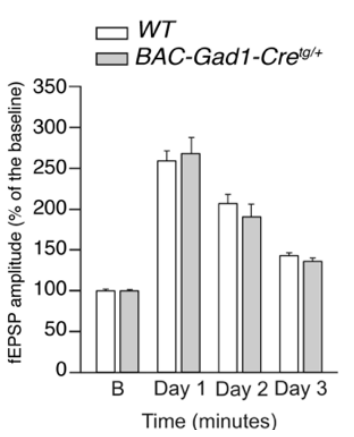

Figure S6. In vivo presynaptic short-term plastic properties are normal at CA3-CA1 synapses in Trkb $^{\text {Gad1-Ko }}$ mice. Related to Figure 6; Table S1. Statistical analysis for data presented in this Figure S6A-B and related to Figure 6; Table S2. Statistical analysis for data presented in Figure 6B-C. Table S3. Statistical analysis for data presented in this Figure S6D-E and related to Figure 6. (A) Paired-pulse facilitation of fEPSPs evoked at the CA3-CA1 synapse following stimulation of Schaffer collaterals. Representative examples of fEPSP paired traces collected for the Trkb ${ }^{\text {Gad1-WT }}$ and $T r k b^{\text {Gadl-KO }}$ groups at $20 \mathrm{~ms}$ and $40 \mathrm{~ms}$ inter-stimulus intervals. Represented values are mean \pm SEM slopes of the second fEPSP expressed as a percentage (\%) of the first for the six inter-stimulus intervals. The two groups presented a significant $(P \leq 0.038)$ increase in the response to the 2 nd pulse at short $(10-40 \mathrm{~ms})$ time intervals, with the largest differences being reached at $40 \mathrm{~ms}$ of inter-pulse intervals. No differences were observed in their facilitation curves $\left[\mathrm{F}_{(5,70)}=0.146, P=0.980 ; \mathrm{n}=8\right.$ animals per group. Two Way Repeated Measures ANOVA, One Factor Repetition]. (B) Similar analysis was carried out for the additional control groups (BAC-Gadl-Cre $e^{t g /+}$ and WT littermates). Also 
in this case, the two groups presented a significant $(P \leq 0.023)$ increase of the response to the 2 nd pulse at short (20-40ms) time intervals, with the largest differences being reached at $40 \mathrm{~ms}$ of inter-pulse intervals, but no differences in their facilitation curves $\left[\mathrm{F}_{(5,70)}=0.916, P=0.476\right.$; $\mathrm{n}=8$ animals per group. Two Way Repeated Measures ANOVA, One Factor Repetition]. (C) Representative examples fEPSP recordings collected from selected animals of each experimental group at times indicated in (D). In all cases, the smaller fEPSP was collected before the HFS (baseline), while the larger one was collected 24-30min after the HFS. (D) Time course of changes in fEPSP slopes (mean \pm SEM) following HFS stimulation of Schaffer collaterals. The HFS was presented after $15 \mathrm{~min}$ of baseline recordings at the time marked by the dashed line. fEPSPs are given as a percentage of baseline (100\%) values. Following the HFS session, fEPSPs were recorded for $30 \mathrm{~min}$ on day 1 , and for $15 \mathrm{~min}$ on the following days. No significant differences were observed between groups $\left[\mathrm{F}_{(24,336)}=1.343 ; P=0.133 ; \mathrm{n}=8\right.$ animals per group. Two Way Repeated Measures ANOVA, One Factor Repetition]. (E) fEPSP data (mean \pm SEM) included in each histogram (B, Baseline; Day 1, 2, 3) were collected for the time intervals indicated in (D). No significant differences were observed between these two groups $\left[\mathrm{F}_{(3,138)}=2.166 ; P=0.095 ; \mathrm{n}=8\right.$ animals per group. Two Way Repeated Measures ANOVA, One Factor Repetition]. 

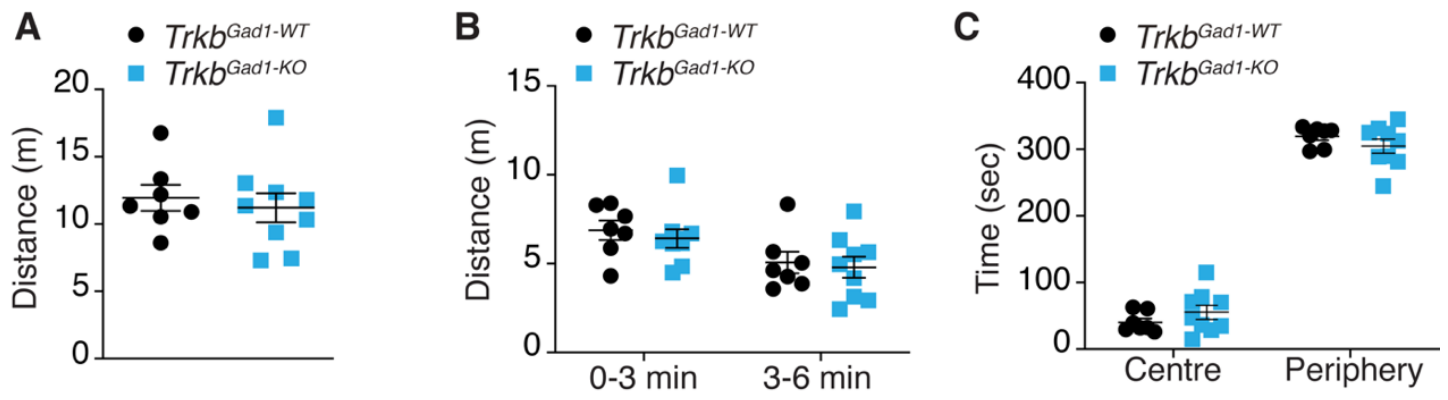

D

Object-context Task

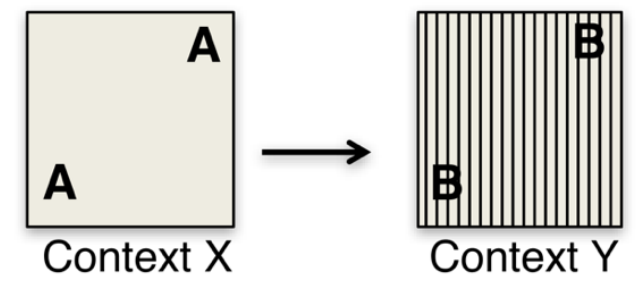

Acquisition, Day 1 to 4

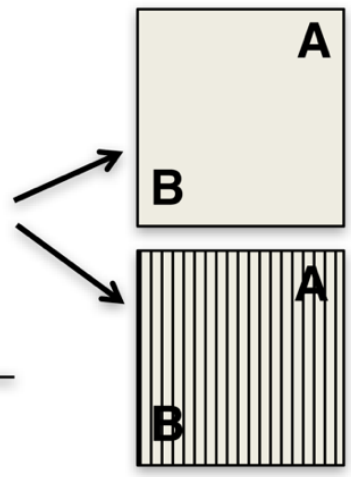

Test

Figure S7. Open field (OF) and object-context recognition (OCR) task. Related to Figure 7. (A) Six-month-old mice were tested in the OF; distance travelled, Trkb $b^{\text {Gadl-WT }}(\mathrm{n}=7)$ and $\operatorname{Trkb}^{\text {Gadl-KO }}(\mathrm{n}=8)$ mice, in a 6-min session of the OF. No difference was found between genotypes (mean distance \pm SEM; Trkb ${ }^{\text {Gadl-WT }}, 11.22 \pm 1.08$; Trkb ${ }^{\text {Gadl-KO }}, 11.96 \pm 0.97$, unpaired Student's $t$-test: $\mathrm{t}_{(14)}=0.4947, P=0.6285$ ). (B) In the OF, both $T r k b$ mutant and control mice travelled a shorter distance in the second half compared to the first half of the 6-min session, suggesting habituation to the arena (Two-way ANOVA, repeated measures, $\mathrm{F}_{(1,14)}=28.88$, $P<0.0092$, main effect of time). (C) $T r k b^{G a d l-K O}$, similar to $\operatorname{Tr} k b^{\text {Gadl-WT }}$ mice, spent less time in the centre compared to the periphery of the open field, suggesting that they were not more anxious than controls (two-way ANOVA; main effect of position: $\mathrm{F}_{(1,28)}=842.9, P<0.0001$ ). (D) OCR test experimental design. The object-context test was performed according to previous studies (Wilson et al., 2013). Acquisition phase, day 1 to day 4, mice were exposed to two copies of object $A$ in context $X$ and two copies of object $B$ in context $Y, 5$ min a day for four days. The order of the exposure to the two contexts was alternated on each day (e.g., X$\mathrm{Y}, \mathrm{Y}-\mathrm{X}, \mathrm{X}-\mathrm{Y}, \mathrm{Y}-\mathrm{X}$ ). Day 5, test, mice received a 3 min test trial in one of the two contexts (depending on the order of their exposure to the contexts during the acquisition phase). A preference for the object that was not previously paired with the context indicated contextdependent object recognition memory. During the test phase, objects consisted of exact copies of those explored during the acquisition phase. The identity of the objects, their location, and the order of test trials in context $\mathrm{X}$ and $\mathrm{Y}$ was fully counterbalanced within each genotype. 
Table S4. Probe sets for Nkcc1 smFISH analysis. Related to Figure 4E-G.

$\begin{array}{ll}\text { Nkcc1 (mouse) } & \text { Probe set name: } \\ & \text { Nkcc1_555 }\end{array}$

Fluorophore: Quasar 570, 548 EX (NM), 566 EM (NM)

\begin{tabular}{|c|c|c|c|}
\hline Count & PROBE PROBE $\left(5^{\prime}>3^{\prime}\right)$ & $\begin{array}{l}\text { PROBE } \\
\text { POSITION * }\end{array}$ & PERCENT GC \\
\hline 48 & 1 tcatagtagtactgctggtg & $\begin{array}{r}\text { POSITION * } \\
568\end{array}$ & $45.00 \%$ \\
\hline & $\begin{array}{l}\text { 1 tcatagtagtactgctggtg } \\
2 \text { aagccatcctcaaaaggttc }\end{array}$ & $\begin{array}{l}500 \\
739\end{array}$ & $\begin{array}{l}4 J .00 \% \\
45.00 \%\end{array}$ \\
\hline & $\angle$ aagccatccicaaaaggitc & 139 & $\begin{array}{l}45.00 \% \\
4000 \%\end{array}$ \\
\hline & 3 atctctggttggagtacttt & 773 & $40.00 \%$ \\
\hline & 4 tacaactcctttactctcgg & 812 & $45.00 \%$ \\
\hline & 5 cggactaatacaccettgat & 844 & $45.00 \%$ \\
\hline & 6 aaagagcatcacaccccaaa & 878 & $45.00 \%$ \\
\hline & 7 ccacgatccatgacaatcta & 900 & $45.00 \%$ \\
\hline & 8 ccattgctattacgacgaca & 942 & $45.00 \%$ \\
\hline & 9 gctgaagtagacaatcctgt & 982 & $45.00 \%$ \\
\hline & 10 caacatacatagcaacggcc & 1116 & $50.00 \%$ \\
\hline & 11 tggaatgttccttaagcagc & 1161 & $45.00 \%$ \\
\hline & 12 cgactgtaatggetccaata & 1215 & $45.00 \%$ \\
\hline & 13 ctatgacgaagtcggcgatg & 1317 & $55.00 \%$ \\
\hline & 14 tccagctagaataccagttg & 1475 & $45.00 \%$ \\
\hline & 15 tatggctgactgaggatctg & 1517 & $50.00 \%$ \\
\hline & 16 catcccgaacaacacacgaa & 1605 & $50.00 \%$ \\
\hline & 17 tgtcagctctgttgtaatgg & 1646 & $45.00 \%$ \\
\hline & 18 gttcattagcccgtaagaac & 1727 & $45.00 \%$ \\
\hline & 19 gacgagagcgtggctgaaaa & 1804 & $55.00 \%$ \\
\hline & 20 gggagcactcacaagagatg & 1832 & $55.00 \%$ \\
\hline & 21 ctgggtagatattgtcctta & 1872 & $40.00 \%$ \\
\hline & 22 tttcccataacctttagcaa & 1904 & $35.00 \%$ \\
\hline & 23 aaccacgaagcggttcatta & 1926 & $45.00 \%$ \\
\hline & 24 gaatcccagtgcaatcagaa & 1958 & $45.00 \%$ \\
\hline & 25 ctccaataagggagatccac & 2121 & $50.00 \%$ \\
\hline & 26 aaatgtacagcccaaggact & 2205 & $45.00 \%$ \\
\hline & 27 agtgcactgaggtaagtcag & 2275 & $50.00 \%$ \\
\hline & 28 ccccagaaagacggattgaa & 2301 & $50.00 \%$ \\
\hline & 29 gagtttggagaacctgtcat & 2362 & $45.00 \%$ \\
\hline & 30 ggcttgatcaatggacatct & 2483 & $45.00 \%$ \\
\hline & 31 catcaagtattgtgcacctt & 2576 & $40.00 \%$ \\
\hline & 32 acaagtgtgtttggcttcat & 2617 & $40.00 \%$ \\
\hline & 33 atatacatatccacatccct & 2674 & $35.00 \%$ \\
\hline & 34 tccacatttactaccacatc & 2827 & $40.00 \%$ \\
\hline & 35 aagcttttggtcagctacat & 2987 & $40.00 \%$ \\
\hline & 36 gaaactgtgtgctagcttca & 3009 & $45.00 \%$ \\
\hline & 37 aagccaccagacatctatag & 3050 & $45.00 \%$ \\
\hline & 38 atgtcaaacctccatcatca & 3072 & $40.00 \%$ \\
\hline
\end{tabular}


39 ataaagtagccatcgctctc

40 atcagagaagtctatccgga

41 ttgatatctccaaggaccat

42 ggctcgatcatgtcatcaaa

43 ttatctgtgattcgccaagg

44 acctgatctgtcgatatgtc

45 gcgactggaagactcatgac

46 ccatatacagagcactggac

47 ttcgagagagcttctaacca

48 gtagaaggtaaggacgctct
3189

3218

3241

3292

3373

3417

3481

3516

3538

3596
$45.00 \%$

$45.00 \%$

$40.00 \%$

$45.00 \%$

$45.00 \%$

$45.00 \%$

$55.00 \%$

$50.00 \%$

$45.00 \%$

$50.00 \%$ 
KEY RESOURCES TABLE

\begin{tabular}{|c|c|c|}
\hline REAGENT or RESOURCE & SOURCE & IDENTIFIER \\
\hline \multicolumn{3}{|l|}{ Antibodies_Biochemistry } \\
\hline$\overline{T r k B}$ & Cell Signaling & $\# 4603$ \\
\hline KCC2 & Upstate & $07-432$ \\
\hline NKCC1 & Sigma-Aldrich & T4-S \\
\hline GAPDH & Sigma-Aldrich & \#G954S \\
\hline HRP Goat anti-rabbit & Stratech Jackson & $111-035-008$ \\
\hline HRP Donkey anti-goat HRP & Stratech Jackson & $705-035-003$ \\
\hline HRP Goat anti-mouse rabbit & Stratech Jackson & $115-035-003$ \\
\hline \multicolumn{3}{|l|}{ Antibodies for immunohistochemistry } \\
\hline Calbindin & Sigma & CB995 \\
\hline Calretinin & Swant & $7699 / 4$ \\
\hline Parvalbumin & Swant & PV-25 \\
\hline Phospho-Creb (Ser133) & Cell Signaling & $87 G 3$ \\
\hline Ki67 & Abcam & $a b 15580$ \\
\hline DCX & Abcam & ab113435 \\
\hline Chicken Anti-GFP & Abcam & ab 13970 \\
\hline Alexa Fluor ${ }^{\circledR} 555$ Donkey anti-rabbit & Invitrogen & A-31572 \\
\hline Alexa Fluor® 555 Donkey anti-mouse & Invitrogen & A-31570 \\
\hline Alexa Fluor® 488 AffiniPure Donkey Anti-Chicken & $\begin{array}{l}\text { Jackson } \\
\text { Immunoresearch }\end{array}$ & $703-545-155$ \\
\hline Biotinylated goat anti-rabbit & Vector Labs & BA-1000 \\
\hline Biotinylated horse anti-rmouse & Vector Labs & BA-2000 \\
\hline \multicolumn{3}{|l|}{ Experimental Models: Organisms/Strains } \\
\hline Trkb-floxed $\left(T r k b^{1 x}\right)$ strain & Minichiello et al., 1999 & $\overline{N A}$ \\
\hline BAC-Gad1-Cre ${ }^{t g /+}$ strain & Ohtsuka et al., 2013 & NA \\
\hline Trkb ${ }^{\text {Gad1-KO }}$ mice & This paper & NA \\
\hline Z/EG reporter strain & Novak et al., 2000 & NA \\
\hline Rosa26-EYFP & Srinivas et al., 2001 & NA \\
\hline Rosa26-Ai9-tdTomato & Jackson Laboratory & Jax Stock\#007909 \\
\hline \multicolumn{3}{|l|}{ Oligonucleotides } \\
\hline smFISH probes for $N k c c 1$ & This paper & Attached as a table \\
\hline & & \\
\hline
\end{tabular}




\section{TRANSPARENT METHODS}

\section{Experimental model and subject details}

All animal procedures conformed to the UK legislation Animals (Scientific Procedures) Act 1986 and to the University of Edinburgh Ethical Review Committee (ERC) policy as well as the University of Oxford ERC policy, and to the National and International laws and policies (EEC Council Directive 86/609, OJ L 358, 1, December 12, 1987; NIH Guide for the Care and Use of Laboratory Animals, NIH Publication No. 85-23, 1985 revised in 1995) procedures at the European Molecular Biology Laboratory (Mouse Biology Unit) Italy, the International School for Advanced Studies (SISSA), Trieste, and European Brain Research Institute (EBRI), Rome, Italy. To European Union Council (2003/65/EU) and Spanish (BOE 252/34367-91, 2005) guidelines for the use of laboratory animals in chronic electrophysiological and behavioural studies as well as the Ethics Committee of the Pablo de Olavide University, Seville, Spain. For all experiments, we used male and female mice, unless stated otherwise, at the age and numbers specified throughout the text.

\section{Mouse strains}

$T r k b^{\text {Gadl-KO }}$ mice, this line derives from a cross between the $\operatorname{Tr} k b$-floxed $\left(\operatorname{Tr} k b^{l x}\right)$ line, previously described (Minichiello et al., 1999), and the BAC-Gad1-Cre ${ }^{t g /+}$ strain, previously described (Ohtsuka et al., 2013). Littermates without the Cre transgene were used as controls $\left(\operatorname{Trkb} b^{l x / x} ; \mathrm{Gadl}-\mathrm{Cre}^{+/+}\right)$unless specified. However, to ensure that the transgene did not carry any phenotype, mice carrying only the transgene (BAC-Gadl-Cre $\left.e^{t g /+}\right)$ and wild-type control littermates were also analysed (see Fig.S6, Table S1, Table S3). Moreover, no differences were found between wild-type and Trkb floxed animals. The reporter lines used were the Z/EG (Novak et al., 2000), the Rosa26-EYFP (Srinivas et al., 2001), and the Rosa26-Ai9-tdTomato (Madisen et al., 2010). All experiments were carried out by an experimenter blind to genotype.

\section{Group allocation}

Littermates were grouped according to their genotypes and then used for all different experiments. Masking was used during group allocation, data collection and data analysis for all electrophysiological and behavioural tests.

\section{In vitro electrophysiology}

Experiments were performed on hippocampal slices from neonatal (P3-P9) and adult (P60P90) mutants and control littermate mice. For neonatal mice, each experiment was replicated minimum 3 times and 3-4 weeks apart (with at least 3 biological replicates and 3 technical replicates). For the adult stage, slices were prepared from 3 to 5 mice for each experimental group. More details are included in the figure legends.

\section{Slice preparation}

Briefly, animals were decapitated and the brain quickly removed from the skull and placed in ice-cold artificial cerebrospinal fluid (aCSF) solution containing (in $\mathrm{mM}$ ): $\mathrm{NaCl} 130, \mathrm{KCl} 3.5$, $\mathrm{NaH}_{2} \mathrm{PO}_{4} 1.2, \mathrm{NaHCO}_{3} 25, \mathrm{MgCl}_{2} 1.3, \mathrm{CaCl}_{2}$ 2, glucose 25 , saturated with $95 \% \mathrm{O}_{2}$ and $5 \%$ 
$\mathrm{CO}_{2}$ (pH 7.3-7.4). Transverse hippocampal slices ( $300 \mu \mathrm{m}$ thick) were cut with a vibratome and stored at room temperature in a holding bath containing the same solution as above. After a recovery period of at least one-hour, an individual slice was transferred to the recording chamber where it was continuously superfused with oxygenated aCSF at a rate of $2-3 \mathrm{ml} / \mathrm{min}$ at $33-34^{\circ} \mathrm{C}$.

\section{Electrophysiological recordings}

In neonatal animals, electrophysiological experiments were performed from visually identified CA3 pyramidal cells using the whole-cell configuration of the patch-clamp technique in current or voltage-clamp mode. Patch electrodes were pulled from borosilicate glass capillaries (Hingelberg, Malsfeld, D). They had a resistance of 4-6 M $\Omega$ when filled with an intracellular solution containing (in $\mathrm{mM}$ ) $\mathrm{KCl} 125, \mathrm{NaCl} 10$, HEPES 10, MgATP 4, Na-GTP 0.4, EGTA 0.5. Recordings were made with a patch clamp amplifier (Multiclamp 700B; Molecular Devices, Sunnyvale, CA, USA). Series resistance compensation was used only for currentclamp recordings. The stability of the patch was checked by repetitively monitoring the input and series resistance during the experiment. Cells exhibiting more than $20 \%$ changes in series resistance were excluded from the analysis. Spontaneously occurring GDPs were recorded in current clamp conditions from a holding potential of $-70 \mathrm{mV}$. Spontaneous and miniature AMPA (sEPSCs) and $\mathrm{GABA}_{\mathrm{A}}$-mediated postsynaptic currents (GPSCs) were recorded in voltage clamp conditions from a holding potential of $-70 \mathrm{mV}$, using the intracellular solution mentioned above. Miniature EPSCs and GPSCs were recorded in the presence of tetrodotoxin (TTX, $1 \mu \mathrm{M}$, purchased from Latoxan, Valence, France). Spontaneous and miniature events were analysed with pClamp 9 (Molecular Devices, Sunnyvale, CA, USA). This program uses a detection algorithm based on a sliding template. The template did not induce any bias in the sampling of events because it was moved along the data trace by one point at a time and was optimally scaled to fit the data at each position. All the collected events were averaged, and the peak of the mean current amplitude was calculated.

The effect of isoguvacine on cells firing was studied in cell-attached recordings. In these cases, the patch pipette was filled with aCSF. Isoguvacine was applied from a puff pipette (intrapipette concentration of isoguvacine, $100 \mu \mathrm{M}$ dissolved in aCSF) positioned close to the patched neurons using a pneumatic PicoPump [PV820 (World Precision Instruments); pulse duration, $0.5-1 \mathrm{~s}$; pressure, 6-8 psi]. To measure the effect of isoguvacine on neuronal firing, we calculated the ratio between the mean firing frequency during 30 s windows preceding and following the application of the drug.

In adult animals, field (f)EPSPs were obtained using a glass microelectrode filled with aCSF placed in the stratum lucidum of the CA3 area. fEPSPs were evoked at the frequency of $0.1 \mathrm{~Hz}$

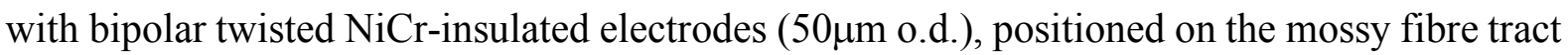
close to the recording electrode. The duration of the stimulus was $80-100 \mu \mathrm{s}$; the stimulation intensity corresponded to that necessary to obtain a response equal to $50 \%$ of the maximal fEPSP. Paired responses evoked 50ms apart were used to measure the paired-pulse ratio (PPR). PPR was calculated as the mean amplitude of the synaptic response evoked by the second stimulus over that evoked by the first one. The input-output curves were constructed by plotting 
the amplitude of the response vs stimulation intensity. LTP was induced by theta burst stimulation of mossy fibres (10 trains, $200 \mathrm{~Hz}, 50 \mathrm{~ms}$ each at a rate of $1 / 100 \mathrm{~ms}$ ). Recordings were obtained with a Multiclamp 700B amplifier connected to a Digidata 1550. Data were acquired with pClamp 9.2 (Molecular Devices), digitised at $10 \mathrm{kHz}$, filtered at $3 \mathrm{kHz}$, and analysed offline with Clampfit 9.2 (Molecular Devices).

\section{Gramicidin-perforated patch recordings}

GABA-mediated postsynaptic currents (GPSCs) evoked in CA3 principal cells by stimulation of MFs in stratum lucidum (in the presence of DNQX and DL-APV to block NMDA and AMPA receptors, respectively) were recorded (from a holding potential of $-70 \mathrm{mV}$ ) using gramicidin perforated patch. In this case, the patch pipette solution contained (in $\mathrm{mM}$ ): 150 $\mathrm{KCl}$ and 10 HEPES, buffered to $\mathrm{pH} 7.2$ with Tris-OH. The extracellular solution contained (in $\mathrm{mM}$ ): $\mathrm{NaCl} 130, \mathrm{KCl} 3.5, \mathrm{NaH} 2 \mathrm{PO} 41.2, \mathrm{NaHCO} 325, \mathrm{MgCl} 21.3, \mathrm{CaCl} 2$ 2, glucose 25, saturated with $95 \% \mathrm{O} 2$ and $5 \% \mathrm{CO} 2(\mathrm{pH} 7.3-7.4)$. Gramicidin was first dissolved in DMSO to prepare a stock solution of $10-40 \mathrm{mg} / \mathrm{ml}$ and then diluted in the pipette solution to a final concentration of $80 \mu \mathrm{g} / \mathrm{ml}$. The gramicidin-containing solution was prepared and sonicated $<1$ $\mathrm{h}$ before the experiment. To facilitate cell-attached formation (4-10G $\Omega$ ), patch pipettes were backfilled with a gramicidin-containing solution. Recordings started 20-30 min after formation of the cell-attached seal and stabilization of the series resistance $(R \mathrm{~s})$ around $60 \mathrm{M} \Omega$. Depolarizing currents were intermittently injected to evoke action potentials to verify the patch integrity, and in the case of membrane rupture, the recording was discontinued. At the end of each recording, negative pressure was applied to break the membrane and establish whole-cell configuration. This was associated with a shift of the reversal potential of the GABA-mediated responses to near $0 \mathrm{mV}$.

Drugs used: 6,7-dinitroquinoxaline-2,3-dione (DNQX), bicuculline (Ascent Scientific), isoguvacine (Sigma-Aldrich), glycine, N-methyl-D-aspartate (NMDA), GABA, SR95531 (Gabazine), DL-2-Amino-5-phosphonopentanoic acid DL-(AP5), L-(+)-2-Amino-4phosphonobutyric acid (L-AP4) (Tocris Bioscience). Gramicidin (Sigma-Aldrich). DNQX was dissolved in dimethylsulphoxide (DMSO). The final concentration of DMSO in the bathing solution was $0.1 \%$. At this concentration, DMSO did not modify the shape or the kinetics of synaptic currents. Drugs were applied in the bath via a three-way tap system, by changing the superfusion solution to one differing only in its content of drug(s). The ratio of flow rate to bath volume ensured full exchange within $2 \mathrm{~min}$. Values are mean $\pm \mathrm{SEM}$.

\section{In vivo LTP}

These experiments were carried out on adult (4 to 5 months old) males. Animals were allowed ad libitum access to chow and water. Eight animals were used for each experimental group. Double-pulse and long-term potentiation (LTP) studies were carried out on eight additional animals per group. Procedure for surgery, recordings and LTP induction were performed according to previous studies (Gruart et al., 2007).

\section{Fear conditioning}


Adult male mice (2-4 months of age) were trained and tested in an operant chamber (18.5 x18 x $21.5 \mathrm{~cm}$ ) (Coulbourn Instruments). The auditory cue (tone) emanated from a loudspeaker located in the sidewall, $15 \mathrm{~cm}$ from the floor. The activity of animals was recorded directly on a computer through a single camera located at the top of the chamber. The presentation of tone and shock stimuli in all training and testing sessions was controlled by GraphicState software (Coulbourn Instruments). Mice were allowed to acclimate to the training chamber for $2 \mathrm{~min}$, and then a tone (CS) of $2800 \mathrm{~Hz}$ frequency and $85 \mathrm{~dB}$ intensity was presented for $30 \mathrm{sec}$ and coterminated in the last $2 \mathrm{sec}$ with a mild footshock $(0.5 \mathrm{~mA})$ [unconditioned stimulus (US)]. Two minutes later, another CS-US pairing was presented, and 30sec later the mice were returned to their home cages. For contextual fear memory, $24 \mathrm{hs}$ later mice were placed back into the same chamber under the same conditions, but this time no tone or foot shock was applied. The time the animals spent without moving for more than $2 \mathrm{sec}$ (freezing behaviour) was measured. Twenty-four hours after the contextual test, mice were tested for cued conditioning in a chamber with altered appearance and odour. The test lasted $6 \mathrm{~min}$, with $2 \mathrm{~min}$ habituation phase and two $1 \mathrm{~min}$ CS-US presentations, spaced by $2 \mathrm{~min}$ inter trial interval (ITI). Number of animals are reported in the figure legend.

\section{Object-context recognition}

This task was performed according to previously published protocols (Wilson et al., 2013). Details of experimental design are reported in Figure S7.

\section{Histology and Immunohistochemistry (IHC)}

Brain tissues were prepared as previously described (Geibel et al., 2014). Free-floating 30 $\mu \mathrm{m}$ brain sections were used for histology and IHC. Sections were chosen from two or three animals for each genotype (as reported in the relevant figure legend) and gave similar results. Representative images are shown. For IF and 3,3'-diaminobenzidine-based immunostainings sections were treated as previously described (Geibel et al., 2014) and probed with specific antibodies (Primary antibodies: Calbindin, Sigma CB995, 1:400; chicken anti-GFP, Abcam (ab 13970), 1:3000; Calretinin, Swant 7699/4, 1:1000; Parvalbumin, Swant PV-25, 1:1000. Phospho-Creb (Ser133), Cell Signalling 87G3 1:1000. Ki67, Abcam ab 15580, 1:5000. DCX, Abcam ab1 13435, 1:250. Secondary antibodies: alexaFluor 555 donkey anti-rabbit, Invitrogen, 1:1000; alexaFluor 555 anti-mouse, Invitrogen, 1:1000; alexaFluor 488 donkey anti-chicken, Jackson Immunoresearch, 1:1000; biotinylated anti-rabbit Vector Labs (BA-1000), 1:200; biotinylated anti-mouse Vector Labs (BA_2000), 1:200.

\section{Immunoblotting}

Mice were sacrificed by cervical dislocation and hippocampi were dissected and snap frozen on dry ice. Tissues were homogenized in 10X volume RIPA buffer $(50 \mathrm{mM}$ Tris, $150 \mathrm{mM} \mathrm{NaCl}$, $1 \%$ Triton X-100, $1 \mathrm{mM}$ EDTA, $0.1 \%$ SDS, $0.5 \%$ sodium deoxycholate) containing protease and phosphatase inhibitors (Thermo Scientific). After mild sonication (5min, cycle: $30 \mathrm{sec}$ On/Off- Bioruptor) the lysates were centrifuged at $4^{\circ} \mathrm{C}, 20000 \mathrm{~g}$ for $20 \mathrm{~min}$ to remove insoluble materials. The concentration of the lysates was measured using the Pierce ${ }^{\mathrm{TM}} \mathrm{BCA}$ Protein Assay Kit. Proteins lysates were treated with loading buffer $(50 \mathrm{mM}$ Tris $\mathrm{pH} 6.8,2 \% \mathrm{SDS}$, 
$10 \%$ glycine, 4M UREA and $100 \mathrm{mM}$ DL-Dithiothreitol (DTT)) at $70^{\circ} \mathrm{C}$ for $10 \mathrm{~min}$, resolved on a 7\% SDS-PAGE gel, and transferred to nitrocellulose filter membranes using the BioRAD apparatus. Membranes were blocked in $2 \%$ fish gelatine and $0.2 \%$ Tween in PBS for $1 \mathrm{hr}$ at room temperature and incubated with primary antibodies (TrkB, Cell Signalling \#4603, 1:500; KCC2, Upstate 07-432, 1:5000; NKCC1, Sigma-Aldrich, T4-S, 1:500; GAPDH, Sigma \#G954S, 1:10000) overnight at $4^{\circ} \mathrm{C}$. After washes in PBS+ 0.1\% Tween (PBS-T) for 30min, secondary antibody (Goat anti-rabbit HRP, Stratech Jackson, 1:5000; Donkey anti-goat HRP, Stratech Jackson, 1:5000; Goat anti-mouse rabbit HRP, Santa-Cruz, 1:5000) was applied for $1 \mathrm{hr}$ at room temperature in blocking buffer. The membranes were washed in PBS-T for 30min and developed using enhanced chemiluminescence (ECL, Santa Cruz) on Hyperfilm ${ }^{\mathrm{TM}}$ ECL (Amersham). To detect KCC2 oligomers, samples were resuspended in 50mM Tris $\mathrm{pH} 6.8$, 2\%SDS, 10\%glycine, 4M UREA and 0.5\% lithium dodecyl sulphate (LDS) to solubilise $\mathrm{KCC} 2$, in non-reducing conditions and warmed at $37^{\circ} \mathrm{C}$ for $10 \mathrm{~min}$. Proteins were resolved by linear 3-8\% Tris-acetate gel system (NuPAGE, Life technologies) and immunoblotted as described above. WBs were performed on 6 animal per group except otherwise indicated in the figure legend. Similar results were obtained on 2 technical replicates and representative images are shown. Densitometry of immunoreactive bands was performed using the Image Studio Lite software (LI-COR sciences).

\section{SmFISH}

Harvested postnatal day seven (P7) brains were embedded in OCT, frozen and stored at $-80^{\circ} \mathrm{C}$ until used. smFISH was performed as described by Stellaris (LGC Biosearch Technologies, Petaluma, CA, USA) RNA FISH frozen tissue protocol, and Lyubimova A, et al. 2013 (Lyubimova et al., 2013).

https://biosearchassets.blob.core.windows.net/assets/bti_stellaris_protocol_frozen_tissue.pdf with minor modifications. Probe libraries were designed to target the coding sequence of Nkccl gene using Stellaris Probe Designer online tool. The library consisted of 48 probes of $20 \mathrm{bps}$ each (Table S4). The probes were coupled to Quasar570 fluorophore. Cryosections $(8 \mu \mathrm{m})$ were collected and directly mounted onto coverslips. Sections were then fixed in $4 \%$ paraformaldehyde for $10 \mathrm{~min}$ at room temperature, followed by $1 \mathrm{hr}$ permeabilization using $70 \%$ ethanol at room temperature. Hybridization with $250 \mathrm{nM}$ fluorescent labelled probes was carried out overnight at $37^{\circ} \mathrm{C}$. Sections were counterstained with DAPI and mounted on slides with Vectashield antifade mounting medium (Vector Laboratories Ltd, Burlingame, CA, USA). Image stacks $(0.2 \mu \mathrm{m}$ distance) were acquired with a Leica microscope equipped with a x100 oil-immersion objective and a Leica DFC 365 FX camera using Leica AF6000 software (Leica, Wetzlar, Germany). Images were processed by a 3D reconstruction software (Leica AF6000) followed by image projection. Three random fields in the CA3 region were imaged for each section ( 2 sections per mouse at CA3 Bregma level 1.95-2.15mm) for a total of 376 cells for control and 398 cells for mutants analysed from 3 mice per genotype. Similar results were obtained on 2 technical replicates and representative images are shown. Quantification of the mRNA dots was done by using StarSearch tool developed by Raj lab (http://rajlab.seas.upenn.edu). DAPI was used to count the number of cells in each field.

\section{Electron microscopy}


Tissue for transmission electron microscopy was prepared as previously described (Wright et al., 2010). Briefly, mice were transcardially perfused with saline solution $(0.9 \% \mathrm{NaCl}, 500$ units heparin per $500 \mathrm{ml}$ solution), followed by $0.1 \mathrm{M}$ PB buffer (pH7.4, EM-grade) containing $4 \%$ paraformaldehyde and $2.5 \%$ glutaraldehyde as previously described. Brains were removed before immersion in fixative solution at room temperature for a further $2 \mathrm{hrs}$. Coronal vibratome sections $(1 \mu \mathrm{m})$ containing the hippocampus were post-fixed in $1 \%$ osmium tetroxide in $0.1 \mathrm{M}$ PB for $45 \mathrm{~min}$ before dehydration through an ascending series of ethanol solutions and propylene oxide. Brain sections were then embedded in Durcupan resin on glass slides. Regions of the hippocampus were then cut out from a randomly selected section using a scalpel and glued onto a resin block for sectioning. Ultrathin sections $(\sim 60 / 70 \mathrm{~nm})$ were cut and collected on formvar-coated grids (Agar Scientific, UK), stained with uranyl acetate and lead citrate in an LKB Ultrostainer and then quantitatively assessed in a Philips CM12 transmission electron microscope (TEM). Standard ultrastructural characteristics were used (Gillingwater et al., 2006) to classify synapses as either symmetrical or asymmetrical. Number of animals are reported in the figure legend.

\section{Timm's stain}

Young adult mice ( 2 months old, $n=3$ per group) were treated according to standard procedure. Briefly, mice were perfused transcardially with sulfide solution $(150 \mathrm{mM} \mathrm{Na} 2 \mathrm{~S}, 60 \mathrm{mM}$ $\mathrm{NaH}_{2} \mathrm{PO}_{4}$ ) for $2 \mathrm{~min}$, then with glutaraldehyde solution (3\% glutaraldehyde, $294 \mathrm{mM} \mathrm{KH}_{2} \mathrm{PO}_{4}$, $1.2 \mathrm{M} \mathrm{Na}_{2} \mathrm{HPO}_{4}, \mathrm{pH} 7.35$ ) for $3 \mathrm{~min}$ followed by sulfide solution for another $2 \mathrm{~min}$. Brains were fixed in $20 \%$ saccharose in glutaraldehyde solution for $24 \mathrm{hrs}$ at $4^{\circ} \mathrm{C}$. Brains were frozen for $5 \mathrm{~min}$ on dry ice, and $40 \mu \mathrm{m}$ cryosections were collected on gelatinized slides and dried at $37^{\circ} \mathrm{C}$ overnight. Slides were incubated in a developer solution (30\% gum arabic, a few crystals of thymol, $121 \mathrm{mM}$ citric acid, $81 \mathrm{mM}$ tri-sodium citrate dihydrate, $154 \mathrm{mM}$ hydrochinon, $5 \mathrm{mM}$ $\mathrm{AgNO}_{3}$ ) at $25^{\circ} \mathrm{C}$ for $30-45 \mathrm{~min}$, rinsed with water, dehydrated in $70 \%, 95 \%$, 2x 100\% ethanol, 2x xylol for $2 \mathrm{~min}$ each and mounted with DEPEC Eukitt.

\section{Golgi-Cox staining}

Two months-old mice (Control: $\mathrm{n}=9$ neurons from 4 mice; mutant: $\mathrm{n}=12$ neurons from 3 mice), were used for these experiments and treated according to a previously published protocol (Gibb and Kolb, 1998).

\section{Dendritic branching and spine density analysis}

Golgi stained neurons from the CA3 and DG hippocampal area were selected for analyses according to the following criteria: (i) full impregnation of the neurons with no apparent dendritic truncation; (ii) presence of at least two primary basilar and one apical dendrite, (iii) each dendrite branched at least once (dendrites arising from the cell body were considered as first-order until they bifurcated into second- order segments and so on). Selected neurons in the respective regions were traced with NeuroLucida, and reconstructed with the software program NeuroLucida Explorer (MicroBrightField Inc.) for automatic measurements of dendritic tree complexity using Sholl analysis. The dendrites complexity was examined by measuring the number of intersections made by dendrites with concentric circles (Sholl rings) drawn at progressive distances $(25 \mu \mathrm{m})$ from the soma. On selected neurons in CA3 and DG, 
the number of dendritic spines was manually counted with the support of NeuroLucida software. Only protrusions with a clear connection of the head of the spine to the shaft of the dendrite were counted as spines. Dendritic spine density was expressed as the ratio number of spines/lengths of the dendritic segment, except first order ones. An experimenter blind to experimental groups performed all analyses.

\section{Statistical analysis}

For the in vitro electrophysiology recordings in neonatal animals, data were sampled at $20 \mathrm{kHz}$ and filtered with a cut-off frequency of $2 \mathrm{kHz}$. Data acquisition was made using pClamp 9 (Axon Instruments, Foster City, CA). Spontaneous and miniature GABAergic and glutamatergic synaptic currents were analysed offline with Clampfit 9 program. They were first collected using the template function of Clampfit and then reviewed by visual inspection. The significance of differences was assessed (using the GraphPad Prism 7) by unpaired Student's $t$-test. A non-parametric test (Wilcoxon signed-rank test) was used when data did not pass the normality test. In adult animals, statistical comparisons were performed by either unpaired Student's $t$-test or one-way ANOVA. For the in vivo LTP experiments, statistical analysis was performed using the SPSS 13.0 for Windows package (SPSS Inc, Chicago, IL). Data were analysed by two-way ANOVA repeated measure (One Factor Repetition). For the OF, OCR tasks, statistical analysis was performed using GraphPad Prism 6. Mean distance in the OF was analysed using two-tailed Student's $t$-test, while habituation to the arena and anxiety in the OF was assessed by two-way ANOVA repeated-measures. Discrimination ratio in the OCR was analysed by two-tailed unpaired Student's $t$-test. Total object exploration was assessed by twoway ANOVA during the acquisition phase, and by two-tailed unpaired Student's $t$-test during the test phase. For the FC, the time freezing during the conditioning phase and the cued FC test were analysed by two-way ANOVA repeated-measures, followed by Sidak's post-hoc analyses. Two-tailed unpaired Student's t-test was used to examine the percentage of time spent freezing evoked by the context. For the immunoblots and the EM, statistical significance was assessed using the unpaired Student's $t$-test. Dendritic length, number of intersections and spine density from Golgi stained neurons were analysed using unpaired Student $t$-test. The significance level (alpha) for all tests was set at 0.05 , and $P$ values were considered significant when $P<0.05$. More details for statistical tests are indicated in the figure legends. Datasets for statistical analysis for the following figures (Figure S6A-B, Figure 6B-C, Figure S6D-E) are attached as Tables (S1-3). 


\section{SUPPLEMENTAL REFERENCES}

Berg, D.A., Su, Y., Jimenez-Cyrus, D., Patel, A., Huang, N., Morizet, D., Lee, S., Shah, R., Ringeling, F.R., Jain, R., et al. (2019). A Common Embryonic Origin of Stem Cells Drives Developmental and Adult Neurogenesis. Cell 177, 654-668 e615.

Geibel, M., Badurek, S., Horn, J.M., Vatanashevanopakorn, C., Koudelka, J., Wunderlich, C.M., Brönneke, H.S., Wunderlich, F.T., and Minichiello, L. (2014). Ablation of TrkB signalling in CCK neurons results in hypercortisolism and obesity. Nat Commun 5, 3427.

Gibb, R., and Kolb, B. (1998). A method for vibratome sectioning of Golgi-Cox stained whole rat brain. J Neurosci Methods 79, 1-4.

Gillingwater, T.H., Ingham, C.A., Parry, K.E., Wright, A.K., Haley, J.E., Wishart, T.M., Arbuthnott, G.W., and Ribchester, R.R. (2006). Delayed synaptic degeneration in the CNS of Wlds mice after cortical lesion. Brain 129, 1546-1556.

Gruart, A., Sciarretta, C., Valenzuela-Harrington, M., Delgado-Garcia, J.M., and Minichiello, L. (2007). Mutation at the TrkB PLC \{gamma\}-docking site affects hippocampal LTP and associative learning in conscious mice. Learn Mem 14, 54-62.

Lyubimova, A., Itzkovitz, S., Junker, J.P., Fan, Z.P., Wu, X., and van Oudenaarden, A. (2013). Single-molecule mRNA detection and counting in mammalian tissue. Nat Protoc 8 , $1743-1758$.

Madisen, L., Zwingman, T.A., Sunkin, S.M., Oh, S.W., Zariwala, H.A., Gu, H., Ng, L.L., Palmiter, R.D., Hawrylycz, M.J., Jones, A.R., et al. (2010). A robust and high-throughput Cre reporting and characterization system for the whole mouse brain. Nat Neurosci 13, 133-140.

Minichiello, L., Korte, M., Wolfer, D., Kühn, R., Unsicker, K., Cestari, V., Rossi-Arnaud, C., Lipp, H.P., Bonhoeffer, T., and Klein, R. (1999). Essential role for TrkB receptors in hippocampus-mediated learning. Neuron 24, 401-414.

Novak, A., Guo, C., Yang, W., Nagy, A., and Lobe, C.G. (2000). Z/EG, a double reporter mouse line that expresses enhanced green fluorescent protein upon Cre-mediated excision. Genesis 28, 147-155.

Ohtsuka, N., Badurek, S., Busslinger, M., Benes, F., Minichiello, L., and Rudolph, U. (2013). GABAergic neurons regulate lateral ventricular development via transcription factor Pax5. Genesis 51(4):234-45.

Srinivas, S., Watanabe, T., Lin, C.S., William, C.M., Tanabe, Y., Jessell, T.M., and Costantini, F. (2001). Cre reporter strains produced by targeted insertion of EYFP and ECFP into the ROSA26 locus. BMC Dev Biol 1, 4.

Wilson, D.I., Langston, R.F., Schlesiger, M.I., Wagner, M., Watanabe, S., and Ainge, J.A. (2013). Lateral entorhinal cortex is critical for novel object-context recognition.

Hippocampus 23, 352-366. 
Wright, A.K., Wishart, T.M., Ingham, C.A., and Gillingwater, T.H. (2010). Synaptic protection in the brain of WldS mice occurs independently of age but is sensitive to genedose. PLoS One 5, e15108. 